\title{
Ant community structure and biological control in Indonesian cacao agroforestry: long-term changes, land-use management and precipitation gradients
}

\author{
Dissertation \\ to obtain the $\mathrm{Ph}$. $\mathrm{D}$. degree \\ in the International Ph. D. Program for Agricultural Sciences in Goettingen (IPAG) \\ at the Faculty of Agricultural Sciences, \\ Georg-August-University Göttingen, Germany
}

presented by

Akhmad Rizali

born in Madiun, Indonesia

Göttingen, December 2012 
1. Name of supervisor: Prof. Dr. Teja Tscharntke

2. Name of co-supervisor: Prof. Dr. Stefan Vidal

Date of dissertation: 17 November 2011 


\section{TABLE OF CONTENTS}

Chapter 1 General introduction 1

$\begin{array}{lr}\text { Biodiversity under global change } & 2\end{array}$

The role of ants in agroecosystems 3

$\begin{array}{ll}\text { Ant diversity in cacao agroforestry } & 4\end{array}$

$\begin{array}{ll}\text { Study area } & 6\end{array}$

$\begin{array}{lr}\text { Chapter outline } & 8\end{array}$

$\begin{array}{lr}\text { References } & 9\end{array}$

Chapter 2 Dissimilarity of ant communities increases with precipitation, but not reduced land-use intensity, in Indonesian cacao agroforestry 15

$\begin{array}{ll}\text { Abstract } & 16\end{array}$

$\begin{array}{ll}\text { Introduction } & 17\end{array}$

$\begin{array}{lc}\text { Methods } & 18\end{array}$

$\begin{array}{ll}\text { Results } & 21\end{array}$

$\begin{array}{ll}\text { Discussion } & 24\end{array}$

$\begin{array}{ll}\text { Conclusions } & 25\end{array}$

$\begin{array}{ll}\text { Acknowledgements } & 26\end{array}$

$\begin{array}{ll}\text { References } & 26\end{array}$

$\begin{array}{ll}\text { Supporting information } & 31\end{array}$

Chapter 3 Long-term change of ant community structure in cacao

agroforestry landscapes in Indonesia $\quad 34$

$\begin{array}{ll}\text { Abstract } & 35\end{array}$

$\begin{array}{ll}\text { Introduction } & 36\end{array}$

$\begin{array}{ll}\text { Methods } & 38\end{array}$ 
Results

Discussion

Conclusions

Acknowledgements

References

Supporting information

Chapter 4 Separating effect of species identity and species richness on ant predation

Abstract

Introduction

Methods

Results

Discussion

Conclusions

Acknowledgements

References

Supporting information

Summary

Acknowledgements

List of publications 
Chapter 1

General introduction 


\section{BIODIVERSITY UNDER GLOBAL CHANGE}

Declining biodiversity due to global change is a major problem on earth. Land-use degradation and climate change are well-known drivers of biodiversity loss (Sala et al., 2000). In the tropics which have the most species-rich and functionally important terrestrial ecosystems (Myers et al., 2000), each year a total of five million hectare of tropical rainforests is lost or degraded to agricultural expansions (Achard et al., 2002). This conversion drives the loss of tropical biodiversity and of many associated ecosystem services (Foley et al., 2005). In addition to this, climate change through temperature increase and reduced precipitation can have an effect on distribution, reproduction and behavior of species (Lovejoy, 2010). Both land-use change and climate change may favor invasive species that are able to adapt to this altered environment (Hellmann et al., 2008).

While the transformation of natural ecosystems is responsible for major losses in species, some agricultural land-use types such as agroforestry systems can conserve biodiversity. Bhagwat et al. (2008) describes major reasons why agroforestry systems can be valuable for biodiversity conservation, i.e. are important for the protection of species and habitats outside formally protected areas and can maintain heterogeneity at the habitat and landscape scales. Further, it has been suggested that shade trees in agroforestry landscapes can reduce pressure on protected forest reserves (Tscharntke et al., 2011). But, as in other agroecosystems, management intensification often negatively affects biodiversity and associated ecosystem functions in agroforests. Intensification by shade tree removal in cacao agroforestry adversely affects biodiversity such as canopy beetles, canopy ants and wasps (Steffan-Dewenter et al., 2007) including predators (Klein et al., 2002). In addition to a decrease in complexity of vegetation structure, frequent pesticide applications accompanying intensive management may also negatively impact 
biodiversity, not only crop plant-feeding species but also non-target organisms including beneficial species such as pollinating bees or predatory ants (Wiktelius et al., 1999).

As the most abundant group of animals on earth, ants face threat of global change. The total number of described ant species is at present over 12,500 , while the total number of species could exceed 25,000 worldwide (Ward, 2010). In terrestrial ecosystem, ants play important roles as scavengers, seeds dispersal and predators (Hölldobler \& Wilson, 1990). The loss, disturbance or alteration of habitat severely affects ant diversity. Fragmentation studies have revealed that ant species richness can be affected even in large remnant rainforest patches (Brühl et al., 2003). Global climate change also affects the distribution of many ant species. Colwell et al. (2008) predict that around $80 \%$ of the ant species of a lowland rainforest could disappear due to elevational range shifts related to temperature increase. As consequence, a lot of ant species will likely go extinct due to climate change (Dunn, 2005; Dunn et al., 2009).

\section{THE ROLE OF ANTS IN AGROECOSYSTEMS}

Ants play an important role as biological control agents of insect pests and fungal diseases in agroecosystems (Majer, 1976; Philpott \& Armbrecht, 2006). Ants reduce undesirable pests in agroecosystems with several mechanisms: (i) directly preying upon pests e.g. the weaver ant (Oecophylla smaragdina) controls mirids by preying on them (Way \& Khoo, 1992), (ii) chemically deterring pests e.g. Anoplolepis gracilipes limits pod weevils by deterring them (McGregor \& Moxon, 1985) and (iii) causing pests to drop from the host plants that they are attacking e.g. Dolichoderus thoracicus (black cocoa ant) controls mirids and cacao pod borers by disturbing them (Khoo \& Chung, 1989; Way \& Khoo, 1992). 
Ants can also reduce fungal phytopathogens by removing spores (de la Fuente \& Marquis, 1999) or restricting interactions between plants and disease vectors (Leston, 1973; Khoo \& Ho, 1992). Dolichoderus thoracicus effectively control the outbreak of fungal diseases (Phytophthora palmivora) on cacao pods by reducing disease transmission (Khoo \& Ho, 1992). However, the presence of certain ant species may also facilitate disease transmission. For example, Technomyrmex albipes (McGregor \& Moxon, 1985) and Philidris cordata (Wielgoss, 2007) play an important role as vector for P. palmivora (black pod diseases) in cacao agroecosystem.

Individual ant species may be effective to control pests and fungal diseases in agroecosystems. In a community context, there are several mechanisms by which ant diversity, rather than identity, may be important. Ants differ substantially in resource use, with some species acquiring more energy from nectar or honeydew, or from different types of arthropods (Philpott et al., 2004). Foraging strategies and recruitment behaviors of ant species also differ (Hölldobler \& Wilson, 1990). Intraspecific differences in foraging or behavior may augment overall responses to herbivore communities as has been shown for spiders (Schmitz \& Suttle, 2001). Similar patterns have been suggested for ants (Philpott \& Armbrecht, 2006) but the evidence is still scarce (Gove, 2007). Therefore, benefits of species diversity in functionality may thus be due to complementarity between species (Armbrecht et al., 2004) with increase in ecosystem service in outcome.

\section{ANT DIVERSITY IN CACAO AGROFORESTRY}

The occurrence of ants in their habitat is always affected by primary stressors such as low temperature, limitations in nest site availability, food supply, microhabitat structure, and resource capture (Andersen, 1995; Andersen, 2000). In addition, interaction between ants and other organisms shape the ant community structure in their habitat (Schultz \& 
McGlynn, 2000; Styrsky \& Eubanks, 2007). Ants interact with plants (as herbivore, nectar consumer, and symbiotic interaction), other animals (as predator, prey and symbionts), and also with fungi (Schultz \& McGlynn, 2000). Interspecific ant interactions cause competitions that shape the structure of ant communities (Hölldobler \& Wilson, 1990).

Interspecific interactions of ants are relevant in agricultural ecosystems. Room (1971) found 128 ant species and 48 genera in $250 \mathrm{~m}^{2}$ area of cacao agroforestry in Ghana. Ecological interactions in combination with physiological and ecological factors, such as type and number of microhabitats (Morrison, 1998), size and type of nesting site (Armbrecht et al., 2004), and food availability (Kaspari et al., 2000) may contribute to allow coexistence of many ant species in very small area of cacao agroforestry.

In cacao agroforestry in Sulawesi, Wielgoss et al. (2010) using a baiting method recorded 160 ant species from 43 cacao agroforestry in Kulawi and Palolo valley differing in distance to nearest primary rainforest (0-2500 m) of the LLNP. Using fogging method, Hosang (2004) found 103 species from 18 sites in Palolo valley and Bos (2006) found 44 species from 12 sites in Kulawi valley. Based on our research, we only found 87 ant species from cacao agroforestry both in Palolo and Kulawi valley.

We noted that the most dominant ant species in cacao agroforestry in Central Sulawesi are A. gracilipes, P. cordata, D. thoracicus and $O$. smaragdina. We also recorded four tramp ant species (human commensal species) from study area i.e. A. gracilipes, T. albipes, Monomorium floricola and Tapinoma melanocephalum (based on McGlynn, 1999). P. cordata is extremely widespread in cacao agroforestry in Central Sulawesi. This species is dominant and invasive (although likely not exotic) which has negative effect on another ant species. Study by Wielgoss et al. (2010) revealed that the presence of $P$. cordata affect ant species richness in cacao agroforestry. 


\section{STUDY AREA}

Ecological research was conducted in cacao agroforestry system in Sulawesi Island, Indonesia (Fig. 1). Sulawesi Island is a key area for cacao production due to the "cacao boom" in 1990s (Potter, 2001) and recently made Indonesia as the world's second largest cacao producer (FAOSTAT, 2010). As consequence, a lot of areas not only of natural and secondary forest but also of perennial crops were replaced with cacao agroforestry. For example in Central Sulawesi, deforestation increase $17 \%$ and area of agroforestry (included cacao) increase 34\% from 1972 to 2002 (Erasmi et al., 2004). At the same time, Sulawesi Island is located in the biogeographical region of Wallacea, which is one of the world's most important hot spots in terms of biodiversity (Myers et al., 2000), with very high rates of endemism (Whitten et al., 2002). This causes concerns that the conversion of primary rainforest habitats severely affects biodiversity in the region. Unfortunately, information about insect diversity in Sulawesi, and especially about ants, is scarce. From 918 ant species described in Indonesia (www.antbase.org), 160 ant species found in cacao agroforestry in Central Sulawesi (Wielgoss et al., 2010).

The study area was comprised of cacao agroforestry landscapes in Central Sulawesi, and included sites with different in land-use intensity and precipitation gradients (Fig. 1, Chapter 2), including sites from a previous project (STORMA, Stability of the Rainforest Margin in Indonesia) (Fig. 1, Chapter 3). From 2000 to 2009, the IndonesianGerman Collaborative Research Center STORMA has established plots to conduct research concentrating on processes and factors of the stability and dynamics of rainforest margins and intensively investigating the Lore Lindu National Park (LLNP) and its buffer zone (www.storma.de). The LLNP is located about 100 kilometres south of Palu, the capital city of Central Sulawesi. The LLNP, an official UNESCO Biosphere Reserve, covers an area of $2,290 \mathrm{~km}^{2}$ with mostly submontane and montane forests and some 
lowland forest remnants with an altitude range of $200-2,610 \mathrm{~m}$. The climate is humid and diurnal with no pronounced seasons. The daytime temperature in lowland areas ranges from $26-32^{\circ} \mathrm{C}$. The annual rainfall ranges from $2000-3000 \mathrm{~mm}$ in the north of the National Park to 3000 - $4000 \mathrm{~mm}$ in the south with a peak from November to March.

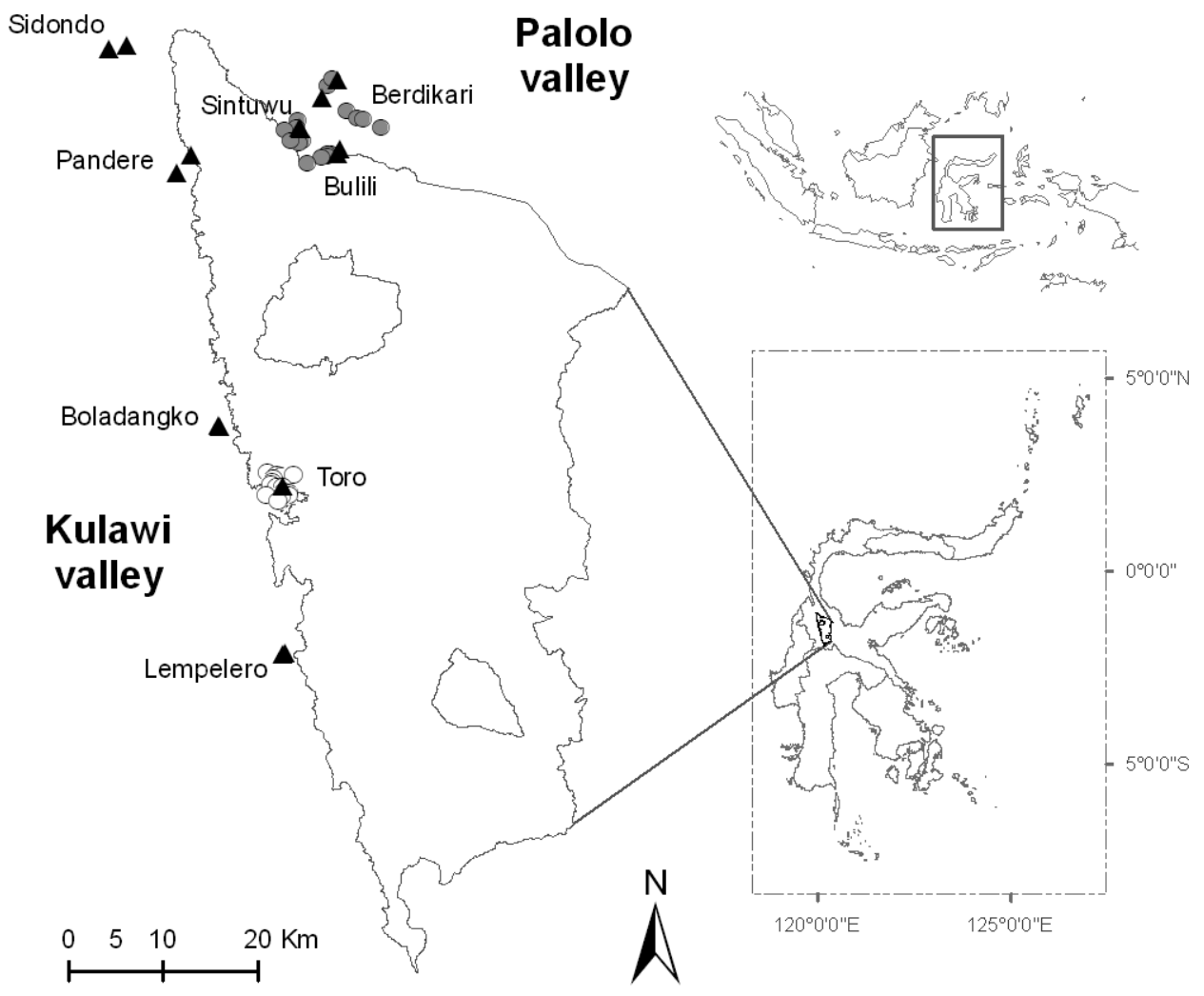

Figure 1. A map of study area in Central of Sulawesi, Indonesia. Study areas were located around the border of the Lore Lindu National Park. Plots with different land-use intensity and precipitation gradients are depicted with solid triangle (chapter 2). Previous plots of STORMA project are depicted with solid and open circle (chapter 3). 


\section{CHAPTER OUTLINE}

Chapter 2 addresses the impact of land-use management and precipitation gradients on ant diversity in cacao agroforestry systems. The research was conducted in cacao agroforestry located around the LLNP. We sampled ants in sixteen plots spread in eight villages, representing different of land-use management and gradients of precipitation, to study the effect of land-use management and precipitation gradients on ant diversity. Our results showed that land-use (canopy cover of shade trees) and precipitation had no effect on species richness of ants per cacao tree (alpha diversity) and, in an additive partitioning approach, within-plot beta diversity. However, higher precipitation (but not shade) significantly decreased community similarity across the cacao trees within a plot, with ant species showing opposing responses to precipitation.

Chapter 3 addresses the changes in ant diversity changes over several years in cacao agroforestry systems. We resampled ants in 2009 with the same method and in the same sites that have been previously studied in 2001 and 2003 within the STORMA (Stability of Rainforest Margins in Indonesia) research project, to study the long-term change of ant diversity in cacao agroforestry. The long-term change in ant communities differed greatly between rainforest-poor (Palolo region) and rainforest-rich (Kulawi region). Temporal changes in ant communities in agroforestry appeared to be more difficult to predict than expected, highlighting the importance of long-term studies for better understanding of the processes underlying the patterns. Our findings suggest that the landscape-scale differences between the two study regions play a more important role than changes in local management. The rainforest-rich region appeared to support a rich species pool with ants colonizing cacao agroforestry and thereby compensating for local land-use intensification. 
In Chapter 4 we investigate the effects of ant species identity and ant community species richness affects pests and diseases in cacao agroforestry systems. To study biological control provided by ants, we conducted field experiment aiming at separating effect of ant species identity and species richness on predation. We found that incidence of the main pest was associated negatively with ant species richness. Ant species richness had no significant effect in improving detection of prey in the predation experiment, and was not associated with a reduction in the incidence of a minor pest. In addition, incidence of the disease caused by $P$. palmivora was higher in ant species rich trees. Identity of the dominant ant species affected incidence of pests and diseases as well as predation pressure.

\section{REFERENCES}

Achard, F., Eva, H.D., Stibig, H.-J., Mayaux, P., Gallego, J., Richards, T. \& Malingreau, J.-P. (2002) Determination of deforestation rates of the world's humid tropical forests. Science, 297, 999-1002.

Andersen, A.N. (1995) A classification of Australian ant communities, based on functional groups which parallel plant life-forms in relation to stress and disturbance. Journal of Biogeography, 22, 15-29.

Andersen, A.N. (2000) Global ecology of rainforest ants: functional groups in relation to environmental stress and disturbance. Ants: Standard Methods for Measuring and Monitoring Biodiversity (ed. by D. Agosti, J.D. Majer, L.E. Alonso and T.R. Schultz), pp. 25-34. Smithsonian Institution Press, Washington.

Armbrecht, I., Perfecto, I. \& Vandermeer, J. (2004) Enigmatic biodiversity correlations: ant diversity responds to diverse resources. Science, 304, 284-286.

Bhagwat, S.A., Willis, K.J., Birks, H.J.B. \& Whittaker, R.J. (2008) Agroforestry: a refuge for tropical biodiversity? Trends in Ecology \& Evolution, 23, 261-267. 
Bos, M.M. (2006) Insect diversity and trophic interactions in shaded cacao agroforestry and natural forests in Indonesia. PhD Thesis, University of Göttingen, Göttingen.

Brühl, C.A., Eltz, T. \& Linsenmair, K.E. (2003) Size does matter - effects of tropical rainforest fragmentation on the leaf litter ant community in Sabah, Malaysia. Biodiversity and Conservation, 12, 1371-1389.

Colwell, R.K., Brehm, G., Cardelús, C.L., Gilman, A.C. \& Longino, J.T. (2008) Global warming, elevational range shifts, and lowland biotic attrition in the wet tropics. Science, 322, 258-261.

de la Fuente, M.A.S. \& Marquis, R.J. (1999) The role of ant-tended extrafloral nectaries in the protection and benefit of a Neotropical rainforest tree. Oecologia, 118, 192-202.

Dunn, R.R. (2005) Modern insect extinctions, the neglected majority. Conservation Biology, 19, 1030-1036.

Dunn, R.R., Agosti, D., Andersen, A.N., Arnan, X., Bruhl, C.A., Cerdá, X., Ellison, A.M., Fisher, B.L., Fitzpatrick, M.C., Gibb, H., Gotelli, N.J., Gove, A.D., Guenard, B., Janda, M., Kaspari, M., Laurent, E.J., Lessard, J.-P., Longino, J.T., Majer, J.D., Menke, S.B., McGlynn, T.P., Parr, C.L., Philpott, S.M., Pfeiffer, M., Retana, J., Suarez, A.V., Vasconcelos, H.L., Weiser, M.D. \& Sanders, N.J. (2009) Climatic drivers of hemispheric asymmetry in global patterns of ant species richness. Ecology Letters, 12, 324-333.

Erasmi, S., Twele, A., Ardiansyah, M., Malik, A. \& Kappas, M. (2004) Mapping deforestation and land-cover conversion at the rainforest margin in Central Sulawesi, Indonesia. EARSeL eProceedings, 3, 388-397.

FAOSTAT (2010) FAO statistical yearbook 2010. UN Food \& Agriculture Organisation.

Foley, J.A., DeFries, R., Asner, G.P., Barford, C., Gordon, B., Carpenter, S.R., Chapin, F.S., Coe, M.T., Daily, G.C., Gibbs, H.K., Helkowski, J.H., Holloway, T., Howard, 
E.A., Kucharik, C.J., Monfreda, C., Patz, J.A., Prentice, I.C., Ramankutty, N. \& Snyder, P.K. (2005) Global consequences of land use. Science, 309, 570-574.

Gove, A.D. (2007) Ant biodiversity and the predatory function (A response to Philpott and Armbrecht, 2006). Ecological Entomology, 32, 435-436.

Hellmann, J.J., Byers, J.E., Bierwagen, B.G. \& Dukes, J.S. (2008) Five potential consequences of climate change for invasive species. Conservation Biology, 22, 534-543.

Hölldobler, B. \& Wilson, E.O. (1990) The Ants. Harvard University Press, Cambridge.

Hosang, M.L.A. (2004) Interactions between natural enemies, herbivores and cacao in Palolo Valley, Central Sulawesi. PhD Thesis, Bogor Agricultural University, Bogor.

Kaspari, M., O'Donnell, S. \& Kercher, J.R. (2000) Energy, density and constraints to species richness: ant assemblages along a productivity gradient. American Naturalist, 155, 280-293.

Khoo, K.C. \& Chung, G.F. (1989) Use of the black cocoa ant to control mirid damage in Cocoa. The Planter (Kuala Lumpur), 65, 370-383.

Khoo, K.C. \& Ho, C.T. (1992) The influence of Dolichoderus thoracicus (Hymenoptera: Formicidae) on losses due to Helopeltis theivora (Heteroptera: Miridae), black pod disease, and mammalian pests in cocoa in Malaysia. Bulletin of Entomological Research, 82, 485-491.

Klein, A.-M., Steffan-Dewenter, I. \& Tscharntke, T. (2002) Predator-prey ratios on cocoa along a land-use gradient in Indonesia. Biodiversity and Conservation, 11, 683-693.

Leston, D. (1973) The ant mosaic - tropical tree crops and the limiting of pests and diseases. International Journal of Pest Management, 19, 311-341. 
Lovejoy, T.E. (2010) Climate change. Conservation Biology for All (ed. by N.S. Sodhi and P.R. Ehrlich), pp. 153-162. Oxford University Press.

Majer, J.D. (1976) The influence of ants and ant manipulation on the cocoa farm fauna. Journal of Applied Ecology, 13, 157-175.

McGlynn, T.P. (1999) The worldwide transfer of ants: geographical distribution and ecological invasions. Journal of Biogeography, 26, 535-548.

McGregor, A.J. \& Moxon, J.E. (1985) Potential for biological control of tent building species of ants associated with Phytophthora palmivora pod rot of cacao in Papua New Guinea. Annals of Applied Biology, 107, 271-277.

Morrison, L.W. (1998) The spatiotemporal dynamics of insular ant metapopulations. Ecology, 79, 1135-1146.

Myers, N., Mittermeier, R.A., Mittermeier, C.G., da Fonseca, G.A.B. \& Kent, J. (2000) Biodiversity hotspots for conservation priorities. Nature, 403, 853-858.

Philpott, S.M. \& Armbrecht, I. (2006) Biodiversity in tropical agroforests and the ecological role of ants and ant diversity in predatory function. Ecological Entomology, 31, 369-377.

Philpott, S.M., Greenberg, R., Bichier, P. \& Perfecto, I. (2004) Impacts of major predators on tropical agroforest arthropods: comparisons within and across taxa. Oecologia, 140, 140-149.

Potter, L. (2001) Agricultural intensification in Indonesia: outside pressures and indigenous strategies. Asia Pacific Viewpoint, 42, 305-324.

Room, P.M. (1971) The relative distributions of ant species in Ghana's cocoa farms. Journal of Animal Ecology, 40, 735-751.

Sala, O.E., Chapin, F.S., Armesto, J.J., Berlow, E., Bloomfield, J., Dirzo, R., HuberSanwald, E., Huenneke, L.F., Jackson, R.B., Kinzig, A., Leemans, R., Lodge, 
D.M., Mooney, H.A., Oesterheld, M., Poff, N.L., Sykes, M.T., Walker, B.H., Walker, M. \& Wall, D.H. (2000) Biodiversity - Global biodiversity scenarios for the year 2100. Science, 287, 1770-1774.

Schmitz, O.J. \& Suttle, K.B. (2001) Effects of top predator species on direct and indirect interactions in a food web. Ecology, 82, 2072-2081.

Schultz, T.R. \& McGlynn, T.P. (2000) The interaction of ants with other organisms. Ants: Standard Methods for Measuring and Monitoring Biodiversity (ed. by D. Agosti, J.D. Majer, L.E. Alonso and T.R. Schultz), pp. 35-44. Smithsonian Institution Press, Washington.

Steffan-Dewenter, I., Kessler, M., Barkmann, J., Bos, M.M., Buchori, D., Erasmi, S., Faust, H., Gerold, G., Glenk, K., Gradstein, S.R., Guhardja, E., Harteveld, M., Hertel, D., Hohn, P., Kappas, M., Kohler, S., Leuschner, C., Maertens, M., Marggraf, R., Migge-Kleian, S., Mogea, J., Pitopang, R., Schaefer, M., Schwarze, S., Sporn, S.G., Steingrebe, A., Tjitrosoedirdjo, S.S., Tjitrosoemito, S., Twele, A., Weber, R., Woltmann, L., Zeller, M. \& Tscharntke, T. (2007) Tradeoffs between income, biodiversity, and ecosystem functioning during tropical rainforest conversion and agroforestry intensification. Proceedings of the National Academy of Sciences, USA, 104, 4973-4978.

Styrsky, J.D. \& Eubanks, M.D. (2007) Ecological consequences of interactions between ants and honeydew-producing insects. Proceedings of the Royal Society B: Biological Sciences, 274, 151-164.

Tscharntke, T., Clough, Y., Bhagwat, S.A., Buchori, D., Faust, H., Hertel, D., Holscher, D., Juhrbandt, J., Kessler, M., Perfecto, I., Scherber, C., Schroth, G., Veldkamp, E. \& Wanger, T.C. (2011) Multifunctional shade-tree management in tropical agroforestry landscapes - a review. Journal of Applied Ecology, 48, 619-629. 
Ward, P.S. (2010) Taxonomy, phylogenetics, and evolution. Ant Ecology (ed. by L. Lach, C.L. Parr and K.L. Abbott), pp. 137-156. Oxford University Press, New York.

Way, M.J. \& Khoo, K.C. (1992) Role of ants in pest-management. Annual Review of Entomology, 37, 479-503.

Whitten, A.J., Mustafa, M. \& Henderson, G.S. (2002) The ecology of Sulawesi. Periplus, Hong Kong.

Wielgoss, A., Tscharntke, T., Buchori, D., Fiala, B. \& Clough, Y. (2010) Temperature and a dominant dolichoderine ant species affect ant diversity in Indonesian cacao plantations. Agriculture, Ecosystems \& Environment, 135, 253-259.

Wielgoss, A.C. (2007) The Impacts of Ants on Pests and Diseases of Cocoa in Indonesian Agroforestry Systems. Universität Würzburg,

Wiktelius, S., Chiverton, P.A., Meguenni, H., Bennaceur, M., Ghezal, F., Umeh, E.-D.N., Egwuatu, R.I., Minja, E., Makusi, R., Tukahirwa, E., Tinzaara, W. \& Deedat, Y. (1999) Effects of insecticides on non-target organisms in African agroecosystems: a case for establishing regional testing programmes. Agriculture, Ecosystems \& Environment, 75, 121-131. 


\section{Chapter 2}

Dissimilarity of ant communities increases with precipitation, but not reduced land-use intensity, in Indonesian cacao agroforestry

Akhmad Rizali, Yann Clough, Damayanti Buchori, Teja Tscharntke 


\begin{abstract}
Land-use degradation and climate change are well-known drivers of biodiversity loss, but little information is available about their potential interaction. Here, we focus on the effects of land-use and precipitation on ant diversity in cacao agroforestry. In Central Sulawesi, Indonesia, we selected 16 cacao agroforestry plots with a shaded vs unshaded plot in each of eight villages differing in precipitation (1032-2051 $\mathrm{mm}$ annual rainfall). On each plot, ten cacao trees with similar size and age (7-10 years) were selected for hand collection of ants on each cacao tree and the soil surface. In total, we found 80 ant species belonging to five subfamilies. Land-use intensification (removal of shade trees) and precipitation had no effect on species richness of ants per cacao tree (alpha diversity) and, in an additive partitioning approach, within-plot beta diversity. However, higher precipitation (but not shade) significantly increased ant community dissimilarity across cacao trees within a plot, with ant species showing opposing responses to precipitation. Reduced precipitation causing drought stress appeared to contribute to convergence of ant community structure, presumably via reduced heterogeneity in cacao tree growth. In conclusion, reduced precipitation greatly influenced ant community dissimilarity and appeared to be more important for ant community structure than land-use change.
\end{abstract}

\title{
Keywords
}

Alpha diversity, beta diversity, community dissimilarity, land-use, precipitation 


\section{INTRODUCTION}

Land-use degradation and climate change are well-known drivers of biodiversity loss (Sala et al., 2000). Temperature increase and reduced precipitation can have an effect on distribution, reproduction and behavior of species (Lovejoy, 2010), and may even favor invasive species that are able to adapt to this altered environment (Hellmann et al., 2008). However, little is known of the interaction of land-use degradation and climate change, although both together may greatly increase biodiversity losses (Tylianakis et al., 2008).

In agricultural ecosystems, land-use intensification exacerbated with climate change effects may severely affect functional biodiversity, for example natural enemies providing successful biological control. Increasing carbon dioxide and temperature can also facilitate the presence of pests and diseases in agricultural habitat and reduce agrobiodiversity (Gornall et al., 2010). In addition, climate change can have direct effect on crop plant growth through increased temperature, drought, rainfall and tropical storms (Gornall et al., 2010).

Agroforestry systems can provide suitable habitat for biodiversity (Bhagwat et al., 2008) and may mitigate effects of climate change (Tscharntke et al., 2011). However, this depends on the management. Agroforestry intensification by shade tree removal (Clough et al., 2011; Tscharntke et al., 2011) and pesticide application (Wanger et al., 2010) has particularly adverse effects on biodiversity. This may have functional consequences, especially if organisms important for pest regulation, such as ants (Philpott \& Armbrecht, 2006), are affected. Bos et al. (2007) found that decreasing shade-tree cover negatively affects ant diversity in cacao agroforestry. Wielgoss et al. (2010) suggest that ant diversity in cacao agroforestry is affected more by temperature than decreasing numbers of shade trees. In general, however, the joint role of climate and shade-tree loss in affecting ant 
species communities has not been addressed, despite its potential significance in ecosystem response to changing rainfall patterns and land-use change.

In this study, we analyze how land-use change and precipitation affect biodiversity in agroforestry. We selected 16 cacao agroforestry plots located in eight villages around Lore Lindu National Park (LLNP) in Central Sulawesi, Indonesia. In each village, we studied one shaded and one unshaded agroforestry system, while the eight villages were situated along a precipitation gradient. We asked the following questions: (i) How does ant community structure change along land-use intensity and precipitation gradients? (ii) Does the ant communities on the cacao tree and on the ground show different responses? (iii) Which ant species show strongest responses to the precipitation gradient?

\section{METHODS}

\section{Study sites}

The 16 studied plots were cacao agroforestry systems located in eight villages around the Lore Lindu National Park (LLNP), Central Sulawesi, Indonesia (Fig. 1). The agroforestry systems differed in land-use intensity (shaded vs unshaded) and the villages in precipitation (1032 to $2051 \mathrm{~mm} /$ year) (Table 1). There is a positive correlation between precipitation and altitude $\left(\mathrm{F}_{1,14}=21.47, \mathrm{P}=0.0004, \mathrm{r}^{2}=0.58\right)$ (see Fig. A). Nevertheless, the better predictor of ant species is precipitation $(\mathrm{df}=5, \mathrm{AIC}=-1.553)$ than altitude $(\mathrm{df}=5$, $\mathrm{AIC}=2.465)$ based on the linear mixed effects model $(\mathrm{LME})$. 


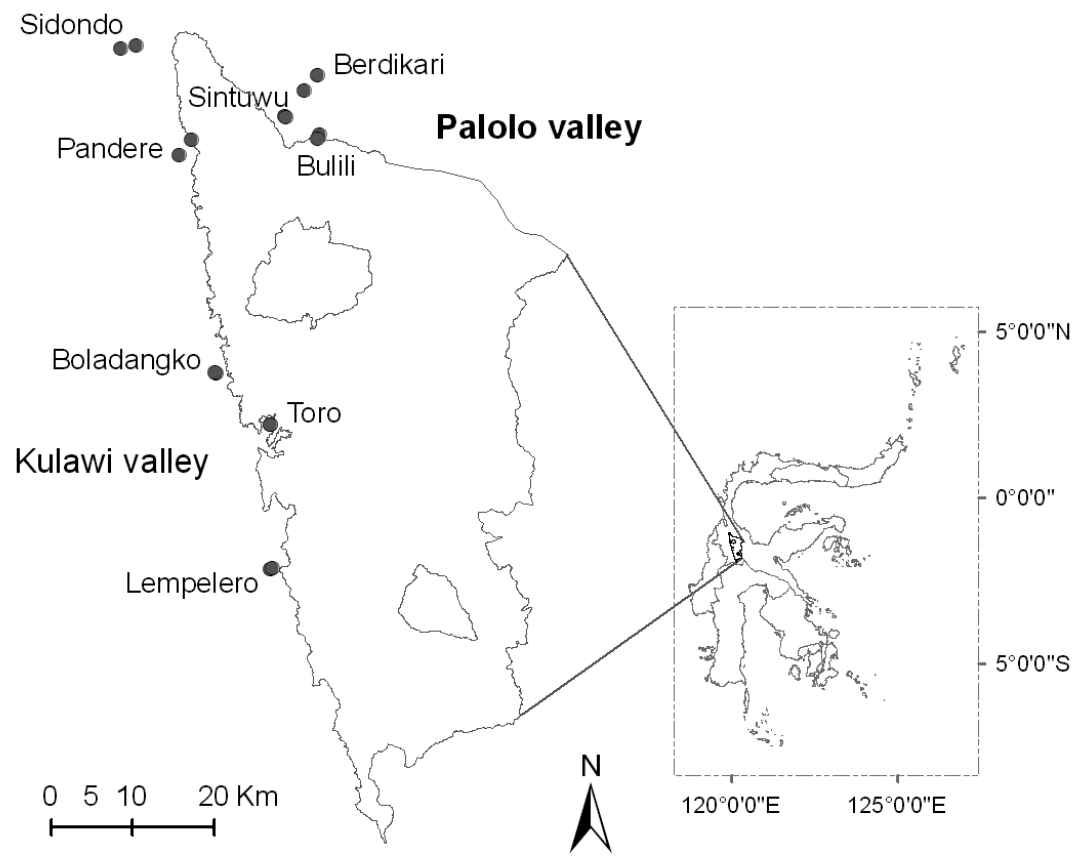

Figure 1. Location map of the 16 cacao agroforestry plots in eight villages with different precipitation levels. Villages are located around Lore Lindu National Park, Central Sulawesi, Indonesia

Table 1. Observed and estimated ant species richness recorded from 16 plots with different landuse (A: shaded; B: unshaded cacao agroforestry) and different precipitation located in 8 villages around Lore Lindu National Park, Central Sulawesi, Indonesia.

\begin{tabular}{|c|l|c|c|c|c|c|c|c|}
\hline No & Village & $\begin{array}{c}\text { Land- } \\
\text { use }\end{array}$ & $\begin{array}{c}\text { Latitude } \\
\left({ }^{\circ} \mathrm{S}\right)\end{array}$ & $\begin{array}{c}\text { Longitude } \\
\left({ }^{\circ} \mathrm{E}\right)\end{array}$ & $\begin{array}{c}\text { Altitude } \\
(\mathrm{m} \text { asl })\end{array}$ & $\begin{array}{c}\text { Precipitation } \\
(\mathrm{mm} / \text { year })\end{array}$ & $\begin{array}{c}\text { Observed } \\
\text { species }\end{array}$ & $\begin{array}{c}\text { Estimated } \\
\text { species }(\%) *\end{array}$ \\
\hline 1. & Berdikari & $\mathrm{A}$ & 01.11693 & 120.09266 & 646 & 2,051 & 24 & $28.5(84.2)$ \\
& & $\mathrm{B}$ & 01.13420 & 120.07861 & 603 & & 17 & $25.0(68.0)$ \\
\hline 2. & Sintuwu & $\mathrm{A}$ & 01.16213 & 120.05642 & 554 & 1,430 & 26 & $32.8(79.4)$ \\
& & $\mathrm{B}$ & 01.16339 & 120.05790 & 567 & & 20 & $52.0(38.5)$ \\
\hline 3. & Bulili & $\mathrm{A}$ & 01.18261 & 120.09550 & 582 & 1,504 & 17 & $29.3(58.1)$ \\
& & $\mathrm{B}$ & 01.18738 & 120.09239 & 652 & & 18 & $22.2(81.2)$ \\
\hline 4. & Pandere & $\mathrm{A}$ & 01.18844 & 119.95393 & 179 & 1,160 & 21 & $23.1(91.0)$ \\
& & $\mathrm{B}$ & 01.20482 & 119.94063 & 102 & & 18 & $19.5(92.3)$ \\
\hline 5. & Sidondo & $\mathrm{A}$ & 01.08366 & 119.89272 & 41 & 1,032 & 23 & $35.3(65.2)$ \\
& & $\mathrm{B}$ & 01.08764 & 119.87599 & 27 & & 16 & $17.5(91.4)$ \\
\hline 6. & Boladangko & $\mathrm{A}$ & 01.44530 & 119.98057 & 523 & 1,590 & 24 & $44.3(54.2)$ \\
& & $\mathrm{B}$ & 01.44499 & 119.98001 & 558 & & 18 & $20.7(87.1)$ \\
\hline 7. & Toro & $\mathrm{A}$ & 01.50238 & 120.04055 & 800 & 1,704 & 26 & $32.4(80.2)$ \\
& & $\mathrm{B}$ & 01.50237 & 120.04109 & 797 & & 21 & $24.1(87.0)$ \\
\hline 8. & Lempelero & $\mathrm{A}$ & 01.66104 & 120.04093 & 432 & 1,742 & 14 & $16.7(84.0)$ \\
& & $\mathrm{B}$ & 01.66035 & 120.04307 & 438 & & 22 & $30.1(73.1)$ \\
\hline
\end{tabular}

* Predicted number of species based on Chao estimator from 10 cacao trees as sampling unit; \%, sampled species as a percentage of predicted number of species 


\section{Plots selection and ant sampling}

Each cacao agroforestry plot differed in agricultural management due to belonged to a different farmer. On each plot, ten cacao trees with similar size and age (7-10 years) were selected for ant observation. Ants were sampled on all strata i.e. the tree trunk and the soil surface using hand collecting which is the best method to record all of ant species inhabiting an area by searching and collecting ants in different microhabitats (Bestelmeyer et al., 2000). Hand collecting was conducted two times in the same cacao trees, in November 2009 and June 2010, to quantify ant species diversity on each cacao tree per plot in different seasons.

Ant specimens were sorted and identified to genus using (Bolton, 1994) or were separated according to their external morphology as morphospecies (Lattke, 2000). Some of the ants could be identified to species level with the help of websites such as http://www.antweb.org.

\section{Data analysis}

The completeness of sampling on each plot was calculated using the Chao estimator (Colwell \& Coddington, 1994). To test for spatial autocorrelation, we used the Mantel test (Mantel, 1967; Legendre \& Legendre, 1998) based on the distance and ant similarity among plots. Alpha diversity was derived from mean ant species richness per tree, whereas beta diversity was calculated based on an additive diversity portioning approach (Gering \& Crist, 2002) with beta diversity resulting from gamma diversity (total ant species in one plot) minus alpha diversity (species richness per tree). The Bray-Curtis dissimilarity index was used to calculate the dissimilarity of ant species communities between the ten cacao tree on each plot, using vegan package in $R$ software ( $R$ Development Core Team, 2011). Then, we calculated the community dissimilarity using 
ordihull (vegan package). Analysis of variance (ANOVA) and linear models (LM) were used to relate ant diversity and community similarity to the land-use and precipitation gradients. Logit regression was performed to analyze the relationship between ant species presence or absence and the precipitation level. All analyses were conducted using $\mathrm{R}$ software (R Development Core Team, 2011).

\section{RESULTS}

\section{Ant community structure along land-use intensity and precipitation gradients}

In total, we found 80 species from 5 subfamilies in the 16 cacao agroforestry plots (see Table A). The Chao estimate for each plot on all 16 sampled cacao agroforestry indicated that these sampling methods captured most of ant species richness (Table 1). Based on the Mantel test, we did not find spatial autocorrelation between similarity of ant communities with nearness distance of plots (Mantel statistic $\mathrm{r}=0.161, \mathrm{P}=0.087$ ). We found that mean ant species richness per tree (alpha diversity), beta diversity (richness per plot minus mean richness per tree) and ant species similarity (on the tree and on the soil surface) across the ten trees per plot were not affected by the presence or absence of shade trees (Table 2). In contrast, precipitation levels significantly affected ant community similarity on the tree (but not on the soil surface) in the cacao agroforestry (Table 2).

According to the linear model, we found that precipitation was not related to the number of ant species richness per tree (alpha diversity, Table 3, Fig. 2a) and beta diversity on the tree (Table 3, Fig. 2b). However, precipitation was closely correlated with the dissimilarity distance among cacao trees on each plot. Increasing precipitation was followed by increasing dissimilarity of ant communities on the tree in the cacao agroforestry systems (Table 3, Fig. 2c). 
Table 2. Effects of shade-tree removal and precipitation on species richness per tree (alpha diversity), beta diversity and community similarity of ant communities in each stratum, based on 16 cacao agroforestry plots.

\begin{tabular}{|l|c|c|c|}
\hline \multirow{2}{*}{ Parameters } & Total & Tree & Soil surface \\
\cline { 2 - 4 } & & & \\
\hline $\begin{array}{l}\text { Alpha diversity } \\
\text { Land-use } \\
\text { Precipitation }\end{array}$ & $\mathrm{F}_{1,13}=0.091, \mathrm{P}=0.768$ & $\mathrm{~F}_{1,13}=0.075, \mathrm{P}=0.788$ & $\mathrm{~F}_{1,13}=0.006, \mathrm{P}=0.941$ \\
\hline $\begin{array}{l}\text { Beta diversity } \\
\text { Land-use }\end{array}$ & $\mathrm{F}_{1,13}=0.003, \mathrm{P}=0.956$ & $\mathrm{~F}_{1,13}=0.449, \mathrm{P}=0.515$ & $\mathrm{~F}_{1,13}=0.057, \mathrm{P}=0.815$ \\
$\quad$ Precipitation & $\mathrm{F}_{1,13}=4.354, \mathrm{P}=0.057$ & $\mathrm{~F}_{1,13}=1.599, \mathrm{P}=0.228$ & $\mathrm{~F}_{1,13}=2.212, \mathrm{P}=0.161$ \\
\hline $\begin{array}{l}\text { Ant community similarity } \\
\text { Land-use } \\
\text { Precipitation }\end{array}$ & $\mathrm{F}_{1,13}=0.197, \mathrm{P}=0.665$ & $\mathrm{~F}_{1,13}=1.807, \mathrm{P}=0.202$ & $\mathrm{~F}_{1,13}=0.124, \mathrm{P}=0.730$ \\
\hline
\end{tabular}

Table 3. Linear model between precipitation and alpha diversity, beta diversity and community similarity in each stratum, based on 16 cacao agroforestry plots

\begin{tabular}{|l|l|l|l|}
\hline \multirow{2}{*}{ Parameters } & \multicolumn{3}{|c|}{ Strata } \\
\cline { 2 - 4 } & \multicolumn{1}{|c|}{ Total } & \multicolumn{1}{c|}{ Tree } & \multicolumn{1}{c|}{ Soil } \\
\hline Alpha diversity & $\mathrm{r}^{2}=-0.071, \mathrm{~F}_{1,14}=0.003$, \\
& $\mathrm{P}=0.954$ & $\begin{array}{l}\mathrm{r}^{2}=-0.036, \mathrm{~F}_{1,14}=0.481, \\
\mathrm{P}=0.450\end{array}$ & $\begin{array}{l}\mathrm{r}^{2}=-0.067, \mathrm{~F}_{1,14}=0.062, \\
\mathrm{P}=0.808\end{array}$ \\
\hline Beta diversity & $\mathrm{r}^{2}=-0.059, \mathrm{~F}_{1,14}=0.159$, & $\begin{array}{l}\mathrm{r}^{2}=0.047, \mathrm{~F}_{1,14}=1.733, \\
\mathrm{P}=0.209\end{array}$ & $\begin{array}{l}\mathrm{r}^{2}=-0.063, \mathrm{~F}_{1,14}=0.114, \\
\mathrm{P}=0.740\end{array}$ \\
& $\mathrm{P}=0.697$ & $\begin{array}{l}\mathrm{r}^{2}=0.280, \mathrm{~F}_{1,14}=6.828, \\
\mathrm{P}=0.021\end{array}$ & $\begin{array}{l}\mathrm{r}^{2}=-0.071, \mathrm{~F}_{1,14}=0.009, \\
\mathrm{P}=0.927\end{array}$ \\
\hline Ant community similarity & $\mathrm{r}^{2}=0.223, \mathrm{~F}_{1,14}=5.296$, & $\mathrm{P}=0.037$ & \multicolumn{2}{|c|}{} \\
\hline
\end{tabular}

(a)

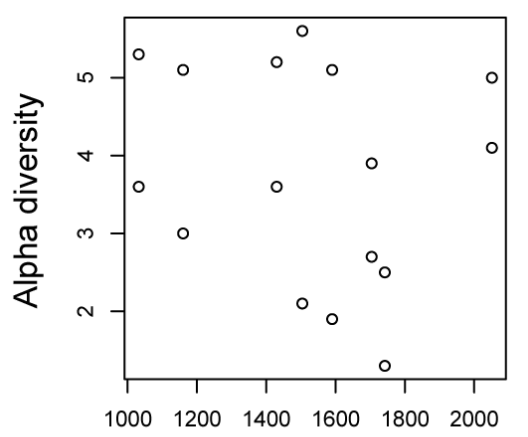

(b)

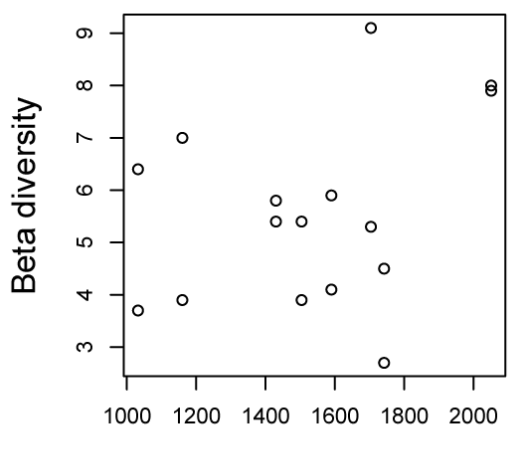

(c)

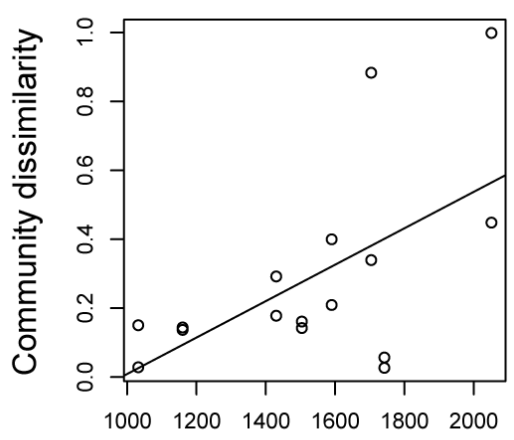

Precipitation

Figure 2. Linear model between precipitation and (a) the mean number species per tree (alpha diversity, $\mathrm{r} 2=-0.036, \mathrm{~F} 1,14=0.481, \mathrm{P}=0.450$ ), (b) beta diversity ( $\mathrm{r} 2=0.047, \mathrm{~F} 1,14=1.733, \mathrm{P}=0.209$ ) and (c) community dissimilarity ( $\mathrm{r} 2=0.280, \mathrm{~F} 1,14=6.828, \mathrm{P}=0.021)$ based on species found on all ten trees per plot. 


\section{Ant species responses to precipitation gradients}

Based on logit regressions for each species, we found that three ant species were related to the precipitation gradients. Crematogaster sp.04 $(\mathrm{df}=1$, Chi-square $=3.84$, $\mathrm{P}=0.04$, Fig 3a) and Tapinoma melanocephalum $(\mathrm{df}=1$, Chi-square $=4.59, \mathrm{P}=0.03$, Fig $3 \mathrm{c})$ was mainly found in cacao agroforestry with low precipitation (Fig 3c). In contrast, Dolichoderus sp.01 was mainly found in cacao plots with high precipitation $(\mathrm{df}=1$, Chisquare $=5.06, \mathrm{P}=0.02$, Fig. $3 \mathrm{~b}$ ). The dominant Dolichoderinae, Philidris cordata, did not show a clear pattern related to precipitation $(\mathrm{df}=1$, Chi-square=2.41, $\mathrm{P}=0.12$, Fig. $3 \mathrm{~d})$.

(a) Crematogaster sp.04

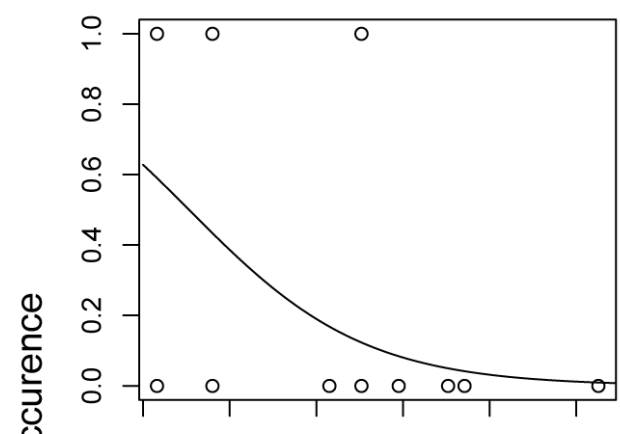

(c) T. melanocephalum

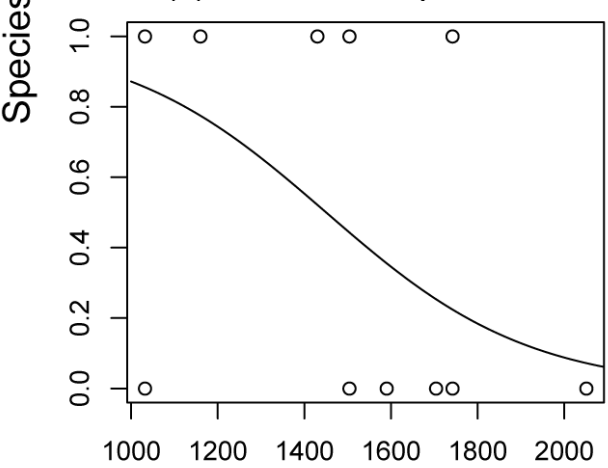

(b) Dolichoderus sp.01

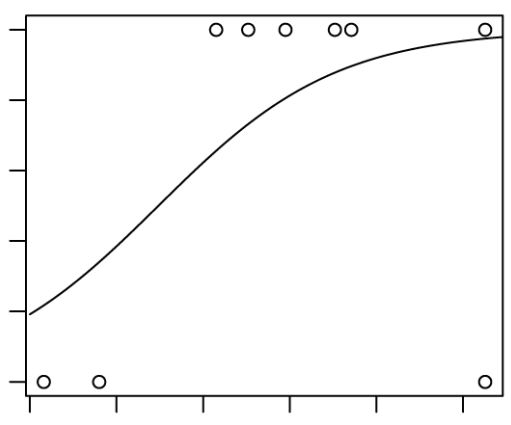

(d) P. cordata

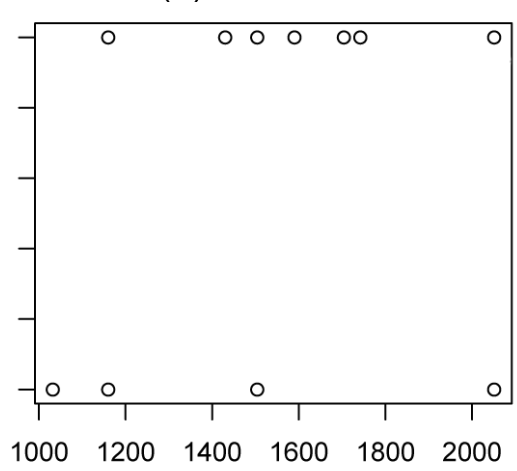

Precipitation

Figure 3. Logit regression of ant species with precipitation. (a) Crematogaster $\mathrm{sp} .04$ ( $\mathrm{df}=1$, Chisquare=3.84, $\mathrm{P}=0.04)$, (b) Dolichoderus sp.01 ( $\mathrm{df}=1$, Chi-square=5.06, $\mathrm{P}=0.02)$, (c) Tapinoma melanochephalum $(\mathrm{df}=1$, Chi-square $=4.59, \mathrm{P}=0.03)$ and $(\mathrm{d})$ Philidris cordata $(\mathrm{df}=1$, Chisquare $=2.41, \mathrm{P}=0.12$ ). 


\section{DISCUSSION}

Our results showed that higher levels of precipitation were associated with higher dissimilarity, but not species richness, of ant communities in cacao agroforestry systems, whereas land-use changes such as losses of shade trees from the cacao agroforestry systems were not related to ant community dissimilarity or diversity. These findings support results of Wielgoss et al. (2010) in the same study region that decreasing shade trees did not affect ant diversity. However, the study by Bos et al. (2007) showed that reduced numbers of shade trees can negatively affect ant diversity, in particular of forest ants, in cacao agroforestry. In the study region of Bos et al. (2007), but not Wielgoss et al. (2010), natural rainforest surrounded their cacao agroforestry systems. Adjacent rainforests (of the national park) provide high forest ant diversity colonizing cacao agroforestry if characterized by high canopy cover from shade-trees. Therefore, shade trees appear to be particularly important when rainforest habitat is surrounding cacao agroforestry as a nearby source of forest ants. In this study, the cacao agroforestry systems were not surrounded by, but rather isolated from rainforests, explaining why shade-tree level was not a predictor of ant diversity.

Precipitation did not affect number of ant species per tree (alpha diversity) in cacao agroforestry. This result is similar to that of Delsinne et al. (2010), who did not find a relationship between precipitation and local ant species richness in arid ecosystems. In our research, the gradient of precipitation was limited (1032 to $2051 \mathrm{~mm} / \mathrm{year}$ ), which may explain why alpha diversity did not show a positive correlation of precipitation and ant diversity (Dunn et al., 2009; Dunn et al., 2010). Nevertheless, we found that increasing precipitation decreased similarity of ant communities in the cacao agroforestry systems. 
High precipitation may influence ant communities because of its negative relationship to ant nesting site availability, which is an important stressor for ants (Andersen, 2000). Nesting site limitation can shape ant community composition, affecting co-existance in addition to factors such as competition, environmental stress and disturbance (Andersen, 1995). In addition, higher precipitation caused higher heterogeneity of ant communities, which should be due to higher heterogeneity of cacao tree growth when drought stress is no longer a dominant and homogenizing factor. Reduced precipitation can be an important stress shaping ant communities in a similar way, i.e. converging ant communities, whereas higher precipitation appeared to allow divergence of ant community structure across trees. Higher precipitation is part of environmental instability affecting the similarity of coexisting species (MacArthur \& Levins, 1967).

The ant species differed in their response to the precipitation level. Dolichoderus sp.01 tended to be mainly found in cacao agroforestry with higher precipitation. Therefore, this species can be expected to suffer most from El Nino (ENSO) droughts, which are common in the region (Gerold \& Leemhuis, 2010). In contrast, T. melanocephalum, a tramp or human commensal species (McGlynn, 1999) and Crematogaster sp.04 were only found in cacao agroforestry with low precipitation, benefiting from droughts. The dominant and invasive species $P$. cordata was not affected by high precipitation and drought, which may be a reason why this species spread widely and became dominant in cacao agroforestry in Sulawesi.

\section{CONCLUSIONS}

Land-use change and precipitation gradients did not affect the ant diversity per cacao tree (alpha diversity) and beta diversity in agroforestry systems in Sulawesi. However, precipitation enhanced ant community similarity, presumably via increased 
heterogeneity in cacao growth with increasing precipitation and subsequent differences in ant colonization, with ant species responding differently to precipitation level. Our results show that changes of precipitation, which are expected to occur in the study region due to El Nino (ENSO) droughts, should contribute to the homogenization of ant community structure.

\section{ACKNOWLEDGEMENTS}

Thanks to our field assistants (Fifin, Moh. Fadlan, Akhmad Syakur and Hadiprayitno) and our laboratory assistants (Nur Ivon and Dewi Utaminingsih). Thanks to the owners of cacao agroforestry in Berdikari (Yosias Nthu and Yosep Sapangan), Sintuwu (Tahana M. Suari and Iskandar), Bulili (Sofyan and Tahang), Pandere (Kunci and Nusrin Abd. Samad), Sidondo (Asran Hadoha and H. Manno), Boladangko (Hamzah and Toruba), Toro (Faldin) and Lempelero (Mursan and Pohabe). Thanks also to the staff of the Center for Tropical Forest Margin (CTFM), University of Tadulako, especially to Pak Abdul Rauf and Mbak Anti who were helping with administration during the research. This research project was funded by the German Science Foundation (DFG), Bogor Agricultural University (IPB), and Directorate General of Higher Education, Ministry of National Education of the Republic of Indonesia.

\section{REFERENCES}

Andersen, A.N. (1995) A classification of Australian ant communities, based on functional groups which parallel plant life-forms in relation to stress and disturbance. Journal of Biogeography, 22, 15-29.

Andersen, A.N. (2000) Global ecology of rainforest ants: functional groups in relation to environmental stress and disturbance. Ants: Standard Methods for Measuring and 
Monitoring Biodiversity (ed. by D. Agosti, J.D. Majer, L.E. Alonso and T.R. Schultz), pp. 25-34. Smithsonian Institution Press, Washington.

Bestelmeyer, B.T., Agosti, D., Alonso, L.E., Brandão, C.R.F., Brown, W.L., Jr., Delabie, J.H.C. \& Silvestre, R. (2000) Field techniques for the study of ground-dwelling ants: an overview, description, and evaluation. Ants: Standard Methods for Measuring and Monitoring Biodiversity (ed. by D. Agosti, J.D. Majer, L.E. Alonso and T.R. Schultz), pp. 122-144. Smithsonian Institution Press, Washington.

Bhagwat, S.A., Willis, K.J., Birks, H.J.B. \& Whittaker, R.J. (2008) Agroforestry: a refuge for tropical biodiversity? Trends in Ecology \& Evolution, 23, 261-267.

Bolton, B. (1994) Identification Guide to the Ant Genera of the World. Harvard University Press, Cambridge.

Bos, M.M., Steffan-Dewenter, I. \& Tscharntke, T. (2007) The contribution of cacao agroforests to the conservation of lower canopy ant and beetle diversity in Indonesia. Biodiversity and Conservation, 16, 2429-2444.

Clough, Y., Barkmann, J., Juhrbandt, J., Kessler, M., Wanger, T.C., Anshary, A., Buchori, D., Cicuzza, D., Darras, K., Putra, D.D., Erasmi, S., Pitopang, R., Schmidt, C., Schulze, C.H., Seidel, D., Steffan-Dewenter, I., Stenchly, K., Vidal, S., Weist, M., Wielgoss, A.C. \& Tscharntke, T. (2011) Combining high biodiversity with high yields in tropical agroforests. Proceedings of the National Academy of Sciences, USA, 108, 8311-8316.

Colwell, R.K. \& Coddington, J.A. (1994) Estimating terrestrial biodiversity through extrapolation. Philosophical Transactions of the Royal Society B: Biological Sciences, 345, 101-118. 
Delsinne, T., Roisin, Y., Herbauts, J. \& Leponce, M. (2010) Ant diversity along a wide rainfall gradient in the Paraguayan dry Chaco. Journal of Arid Environments, 74, 1149-1155.

Dunn, R.R., Guenard, B., Weiser, M.D. \& Sanders, N.J. (2010) Geographic gradients. Ant Ecology (ed. by L. Lach, C.L. Parr and K.L. Abbott), pp. 38-58. Oxford University Press, New York.

Dunn, R.R., Agosti, D., Andersen, A.N., Arnan, X., Bruhl, C.A., Cerdá, X., Ellison, A.M., Fisher, B.L., Fitzpatrick, M.C., Gibb, H., Gotelli, N.J., Gove, A.D., Guenard, B., Janda, M., Kaspari, M., Laurent, E.J., Lessard, J.-P., Longino, J.T., Majer, J.D., Menke, S.B., McGlynn, T.P., Parr, C.L., Philpott, S.M., Pfeiffer, M., Retana, J., Suarez, A.V., Vasconcelos, H.L., Weiser, M.D. \& Sanders, N.J. (2009) Climatic drivers of hemispheric asymmetry in global patterns of ant species richness. Ecology Letters, 12, 324-333.

Gering, J.C. \& Crist, T.O. (2002) The alpha-beta-regional relationship: providing new insights into local-regional patterns of species richness and scale dependence of diversity components. Ecology Letters, 5, 433-444.

Gerold, G. \& Leemhuis, C. (2010) Effects of "ENSO-events" and rainforest conversion on river discharge in Central Sulawesi (Indonesia). Tropical Rainforests and Agroforests under Global Change: Ecological and Socio-economic Valuations (ed. by T. Tscharntke, C. Leuschner, E. Veldkamp, H. Faust, E. Guhardja and A. Bidin), pp. 327-350. Springer-Verlag, Berlin.

Gornall, J., Betts, R., Burke, E., Clark, R., Camp, J., Willett, K. \& Wiltshire, A. (2010) Implications of climate change for agricultural productivity in the early twenty-first century. Philosophical Transactions of the Royal Society of London, Series B: Biological Sciences, 365, 2973-2989. 
Hellmann, J.J., Byers, J.E., Bierwagen, B.G. \& Dukes, J.S. (2008) Five potential consequences of climate change for invasive species. Conservation Biology, 22, 534-543.

Lattke, J.E. (2000) Specimen processing: building and curating an ant collection. Ants: Standard Methods for Measuring and Monitoring Biodiversity (ed. by D. Agosti, J.D. Majer, L.E. Alonso and T.R. Schultz), pp. 155-171. Smithsonian Institution Press, Washington.

Legendre, P. \& Legendre, L. (1998) Numerical ecology 2nd English Edition. Elsevier, The Netherlands.

Lovejoy, T.E. (2010) Climate change. Conservation Biology for All (ed. by N.S. Sodhi and P.R. Ehrlich), pp. 153-162. Oxford University Press.

MacArthur, R. \& Levins, R. (1967) The limiting similarity, convergence, and divergence of coexisting species. American Naturalist, 101, 377-385.

Mantel, N. (1967) The detection of disease clustering and a generalized regression approach. Cancer Research, 27, 209-220.

McGlynn, T.P. (1999) The worldwide transfer of ants: geographical distribution and ecological invasions. Journal of Biogeography, 26, 535-548.

Philpott, S.M. \& Armbrecht, I. (2006) Biodiversity in tropical agroforests and the ecological role of ants and ant diversity in predatory function. Ecological Entomology, 31, 369-377.

R Development Core Team (2011) R: A language and environment for statistical computing. R Foundation for Statistical Computing.

Sala, O.E., Chapin, F.S., Armesto, J.J., Berlow, E., Bloomfield, J., Dirzo, R., HuberSanwald, E., Huenneke, L.F., Jackson, R.B., Kinzig, A., Leemans, R., Lodge, D.M., Mooney, H.A., Oesterheld, M., Poff, N.L., Sykes, M.T., Walker, B.H., 
Walker, M. \& Wall, D.H. (2000) Biodiversity - Global biodiversity scenarios for the year 2100. Science, 287, 1770-1774.

Tscharntke, T., Clough, Y., Bhagwat, S.A., Buchori, D., Faust, H., Hertel, D., Holscher, D., Juhrbandt, J., Kessler, M., Perfecto, I., Scherber, C., Schroth, G., Veldkamp, E. \& Wanger, T.C. (2011) Multifunctional shade-tree management in tropical agroforestry landscapes - a review. Journal of Applied Ecology, 48, 619-629.

Tylianakis, J.M., Didham, R.K., Bascompte, J. \& Wardle, D.A. (2008) Global change and species interactions in terrestrial ecosystems. Ecology Letters, 11, 1351-1363.

Wanger, T.C., Rauf, A. \& Schwarze, S. (2010) Pesticides and tropical biodiversity. Frontiers in Ecology and the Environment, 8, 178-179.

Wielgoss, A., Tscharntke, T., Buchori, D., Fiala, B. \& Clough, Y. (2010) Temperature and a dominant dolichoderine ant species affect ant diversity in Indonesian cacao plantations. Agriculture, Ecosystems \& Environment, 135, 253-259. 


\section{SUPPORTING INFORMATION}

\section{Appendix Table}

Table A. The ant species diversity (proportion of trees occupied, 10 cacao trees observed) collected in the 16 cacao agroforestry plots in eight villages (1: Berdikari, 2: Sintuwu, 3: Bulili, 4: Pandere, 5: Sidondo, 6: Boladangko, 7: Toro, and 8: Lempelero) with different land-use (A: shaded, B: unshaded cacao agroforestry)

\begin{tabular}{|c|c|c|c|c|c|c|c|c|c|c|c|c|c|c|c|c|c|}
\hline \multirow{3}{*}{ No } & \multirow{3}{*}{ Species } & \multicolumn{16}{|c|}{ Village } \\
\hline & & \multicolumn{2}{|c|}{1} & \multicolumn{2}{|c|}{2} & \multicolumn{2}{|c|}{3} & \multicolumn{2}{|c|}{4} & \multicolumn{2}{|c|}{5} & \multicolumn{2}{|c|}{6} & \multicolumn{2}{|c|}{7} & \multicolumn{2}{|c|}{8} \\
\hline & & $\mathbf{A}$ & B & $\mathbf{A}$ & B & $\mathbf{A}$ & B & $\mathbf{A}$ & B & $\mathbf{A}$ & B & $\mathbf{A}$ & B & A & B & A & B \\
\hline \multicolumn{18}{|c|}{ Dolichoderinae } \\
\hline 1. & Dolichoderus sp.01 & & 0.5 & 0.4 & 0.5 & 0.2 & 0.7 & & & & & 0.9 & 0.2 & 0.5 & 0.2 & 0.1 & 0.2 \\
\hline 2. & Dolichoderus thoracicus & & & & & & 1 & & & 1 & & & & & & & \\
\hline 3. & Philidris cordata & & 0.7 & 1 & 1 & 1 & & 1 & & & & 0.7 & 1 & 0.9 & 1 & 1 & 1 \\
\hline 4. & Tapinoma melanocephalum & & & 0.2 & 0.7 & & 0.3 & 0.3 & 0.9 & 1 & & & & & & & 0.2 \\
\hline 5. & Tapinoma sp.01 & 0.4 & 1 & 0.2 & & & & 0.7 & & & 0.9 & 0.1 & 0.7 & 0.8 & 0.7 & & 0.2 \\
\hline 6. & Technomyrmex albipes & & & 0.3 & & & 1 & & & & & 0.9 & & 0.2 & & & \\
\hline 7. & Technomyrmex sp.01 & & & & & & & & & & & & & 0.3 & & & \\
\hline \multicolumn{18}{|c|}{ Formicinae } \\
\hline 8. & Anoplolepis gracilipes & & 0.1 & & & & & & 0.5 & & 1 & 0.8 & 0.3 & 0.5 & 0.4 & & \\
\hline 9. & Camponotus recticulatus & & 0.4 & 0.9 & 0.2 & 0.7 & 0.1 & 0.1 & & & & 0.3 & 0.6 & 0.1 & & & 0.5 \\
\hline 10. & Camponotus sp.01 & 0.9 & & & 0.1 & 0.1 & 0.1 & 0.1 & 0.1 & & & & 0.1 & 0.1 & & & 0.1 \\
\hline 11. & Camponotus sp.02 & & & & 0.1 & & & & & & & & & & & & \\
\hline 12. & Echinopla lineata & 0.1 & & 0.8 & & 0.5 & & & & & & & & & & & \\
\hline 13. & Oecophylla smaragdina & & 0.1 & 0.2 & 0.1 & & & & 1 & & & & & & & & \\
\hline 14. & Paratrechina longicornis & & & & & & & 1 & & & & & & & & & 0.4 \\
\hline 15. & Paratrechina sp.01 & 1 & & 0.9 & 1 & 1 & 1 & 0.6 & 1 & 0.8 & & 1 & 0.8 & 1 & 1 & 1 & 0.9 \\
\hline 16. & Paratrechina sp.02 & & 1 & & & & & & & & & & & & & & \\
\hline 17. & Paratrechina sp.03 & & & & & & & & & 0.7 & & & & & & & \\
\hline 18. & Paratrechina sp.04 & 0.1 & 0.8 & & & & & 0.3 & & & 1 & & & & & & \\
\hline 19. & Plagiolepis sp.01 & 0.9 & & & & & & & & & & & & & & & \\
\hline 20. & Polyrachis abdominalis & 0.3 & & & & & & & & 0.2 & & & & & & & \\
\hline 21. & Polyrachis dives & & & 0.7 & 0.5 & 0.3 & & 0.2 & & & & & & 0.2 & & & 0.1 \\
\hline 22. & Polyrachis sp.01 & & & 0.3 & 0.4 & & & & & & & & & & & & \\
\hline 23. & Polyrachis sp.02 & & & 0.5 & & & & & & & & & 0.4 & & & & \\
\hline 24. & Polyrachis sp.03 & 0.1 & & & & & & & & & & & & & & & \\
\hline 25. & Polyrachis sp.04 & & & & & 0.2 & & & & & & & & & & & \\
\hline 26. & Polyrachis sp.05 & 0.8 & & & & & & & & & & & & & & & \\
\hline 27. & Polyrachis sp.06 & & & 0.1 & & & & & & & & & & 0.2 & 0.1 & & \\
\hline 28. & Polyrachis sp.07 & 0.4 & & & & & & & & & & & & & & & \\
\hline 29. & Pseudolasius sp.01 & & & & & & & & & & & 0.2 & & 0.3 & 0.1 & & \\
\hline 30. & Pseudolasius sp.02 & & & & & & & & & & & & & & 0.3 & & \\
\hline
\end{tabular}


Chapter 2 - Dissimilarity of ant communities

\begin{tabular}{|c|c|c|c|c|c|c|c|c|c|c|c|c|c|c|c|c|c|}
\hline \multirow{3}{*}{ No } & \multirow{3}{*}{ Species } & \multicolumn{16}{|c|}{ Village } \\
\hline & & \multicolumn{2}{|c|}{1} & \multicolumn{2}{|c|}{2} & \multicolumn{2}{|c|}{3} & \multicolumn{2}{|c|}{4} & \multicolumn{2}{|c|}{5} & \multicolumn{2}{|c|}{6} & \multicolumn{2}{|c|}{7} & \multicolumn{2}{|c|}{8} \\
\hline & & $\mathbf{A}$ & B & $\mathbf{A}$ & B & $\mathbf{A}$ & B & $\mathbf{A}$ & B & $\mathbf{A}$ & B & $\mathbf{A}$ & B & $\mathbf{A}$ & B & $\mathbf{A}$ & B \\
\hline \multicolumn{18}{|c|}{ Myrmicinae } \\
\hline 31. & Crematogaster sp.01 & 0.6 & & & & & & 0.1 & 0.7 & & 0.6 & & & & & & \\
\hline 32. & Crematogaster sp.02 & & 0.6 & 0.1 & & 0.1 & 0.2 & 0.2 & 0.6 & 0.1 & 0.1 & 0.7 & 0.3 & 0.2 & 0.8 & 0.2 & 0.6 \\
\hline 33. & Crematogaster sp.03 & & & 0.2 & & & & & & & & & & & & & \\
\hline 34. & Crematogaster sp.04 & & & & & & 0.2 & & 0.9 & 0.8 & & & & & & & \\
\hline 35. & Crematogaster $\mathrm{sp} .05$ & & & & & & & & & & & & & 0.3 & 0.1 & & \\
\hline 36. & Monomorium floricola & & 0.7 & & & & 0.7 & & 1 & 0.3 & 1 & 0.3 & 0.2 & 0.1 & & & \\
\hline 37. & Monomorium sp.01 & & & & & & & & 0.2 & 0.1 & 0.2 & & & & & & \\
\hline 38. & Monomorium sp.02 & 0.4 & & & & & & & & & & 0.1 & & & & 0.3 & \\
\hline 39. & Monomorium sp.03 & & & & & & & 0.2 & & & & & & & & & \\
\hline 40. & Monomorium sp.04 & & 0.1 & & & & & & & & 0.1 & & & & & & \\
\hline 41. & Monomorium sp.05 & & & & & 0.1 & 0.2 & & & & & & & & & & \\
\hline 42. & Monomorium sp.06 & & & & & & & & & & 0.1 & & & & & 0.1 & \\
\hline 43. & Pheidole sp.01 & 0.3 & 0.7 & 0.1 & 0.3 & 0.3 & 0.3 & 0.7 & 0.7 & 0.7 & 0.6 & 0.3 & & & & 0.5 & 0.2 \\
\hline 44. & Pheidole sp.02 & 0.9 & & & & 0.1 & 0.1 & 0.1 & & & & 0.1 & & & 0.6 & 0.4 & 0.1 \\
\hline 45. & Pheidole sp.03 & 0.4 & 0.9 & & & & & 0.7 & & 0.7 & 0.5 & 0.1 & 0.4 & 0.1 & 0.3 & 0.1 & \\
\hline 46. & Pheidole sp.04 & & & 0.1 & & & & & & & & & & & & & \\
\hline 47. & Pheidole sp.05 & & & & 0.5 & & & & & & & 0.1 & & & 0.6 & & \\
\hline 48. & Pheidole sp.06 & 0.1 & & & 0.4 & 0.1 & & & & & & 0.1 & & 0.2 & 0.2 & 0.2 & \\
\hline 49. & Pheidole sp.07 & & & & & & & & & & & & & & & & 0.1 \\
\hline 50. & Pheidole sp.08 & & & 0.1 & 0.1 & & & & & & & & & & & & \\
\hline 51. & Pheidologeton sp.01 & & & & & & & & & & & 0.1 & & & & & \\
\hline 52. & Pyramica paradoxa & & & & & & & & & 0.1 & & & & & & & \\
\hline 53. & Solenopsis geminata & & & & & & & & & 0.4 & & & & & & & \\
\hline 54. & Strumigenys sp.01 & & & & & & & & & 0.1 & & & 0.1 & & 0.1 & & 0.1 \\
\hline 55. & Tetramorium bicarinatum & & 0.9 & & & & & & & 0.1 & 0.4 & & & & & & \\
\hline 56. & Tetramorium pasificum & 0.2 & & & & & 0.9 & & & & & 0.5 & & 0.3 & & & \\
\hline 57. & Tetramorium smithi & & & & & & 0.4 & & & & 0.3 & & 0.1 & 0.1 & 0.1 & & 0.1 \\
\hline 58. & Tetramorium sp.01 & & & & & & & & 0.5 & & & & & & & & \\
\hline 59. & Tetramorium sp.02 & & 0.2 & & & & & & & & 0.2 & & & & & & \\
\hline 60. & Tetramorium sp.03 & 0.2 & & & & & & & & 0.3 & & & & & & & \\
\hline 61. & Tetramorium sp.04 & 0.4 & & & & 0.1 & & & & 0.1 & & & & & & & \\
\hline 62. & Tetramorium sp.05 & & & 0.1 & 0.1 & & & & & 0.3 & & & 0.1 & 0.1 & & 0.1 & 0.1 \\
\hline 63. & Tetramorium sp.06 & & & & & & & & & & & 0.3 & & 0.4 & & & 0.1 \\
\hline 64. & Tetramorium sp.07 & & & & & & & & & & & & & 0.3 & & & \\
\hline 65. & Tetramorium sp.08 & & & 0.2 & 0.1 & & & & & & & & & & & & \\
\hline \multicolumn{18}{|c|}{ Ponerinae } \\
\hline 66. & Anochetus graeffei & & & & & & 0.3 & 0.3 & 0.4 & 0.2 & & & 0.3 & & 0.2 & & \\
\hline 67. & Diacamma rugosum & & & & & & & 0.4 & 0.1 & 0.6 & 0.9 & & & & & & \\
\hline 68. & Hypoponera sp.01 & 0.2 & & 0.1 & 0.1 & & & 0.2 & & & & 0.1 & & & 0.2 & & \\
\hline 69. & Hypoponera sp.02 & & & 0.8 & 0.9 & 0.3 & 0.1 & & & & & 0.6 & 0.8 & 0.9 & 0.5 & 0.7 & 0.3 \\
\hline 70. & Hypoponera sp.03 & & & 0.1 & & & & & & 0.4 & & 0.2 & & & & & \\
\hline 71. & Hypoponera sp.04 & & & & & & & & 0.2 & & & & & & & & 1 \\
\hline 72. & Leptogenys sp.01 & 0.1 & & 0.2 & & & & & & & & & & 0.1 & 0.3 & & \\
\hline 73. & Odontomachus simillimus & 0.2 & 0.5 & 0.7 & 0.7 & 1 & & 0.1 & 0.4 & 0.3 & & 0.7 & 0.3 & 1 & 0.9 & 1 & 0.2 \\
\hline 74. & Pachycondyla sp.01 & 0.4 & & & & & 0.1 & 0.2 & & & 0.2 & & & 0.1 & & 0.2 & 0.4 \\
\hline 75. & Pachycondyla sp.02 & 0.1 & & & & & & & & & & & & & & & \\
\hline 76. & Pachycondyla sp.03 & & & & & & & & 0.2 & & & & & & & & \\
\hline
\end{tabular}




\begin{tabular}{|c|c|c|c|c|c|c|c|c|c|c|c|c|c|c|c|c|c|}
\hline \multirow{3}{*}{ No } & \multirow{3}{*}{ Species } & \multicolumn{16}{|c|}{ Village } \\
\hline & & \multicolumn{2}{|c|}{1} & \multicolumn{2}{|c|}{2} & \multicolumn{2}{|c|}{3} & \multicolumn{2}{|c|}{4} & \multicolumn{2}{|c|}{5} & \multicolumn{2}{|c|}{6} & \multicolumn{2}{|c|}{7} & \multicolumn{2}{|c|}{8} \\
\hline & & $\mathbf{A}$ & B & $\mathbf{A}$ & B & $\mathbf{A}$ & B & $\mathbf{A}$ & B & $\mathbf{A}$ & $\mathbf{B}$ & $\mathbf{A}$ & B & $\mathbf{A}$ & $\mathbf{B}$ & $\mathbf{A}$ & $\mathbf{B}$ \\
\hline 77. & Pachycondyla sp.04 & & & & & & & & & 0.1 & & & & & & & \\
\hline 78. & Platythyrea sp.01 & & & 0.1 & 0.1 & 0.1 & & 0.2 & 0.1 & & & 0.1 & & & & & \\
\hline \multicolumn{18}{|c|}{ Pseudomyrmicinae } \\
\hline 79. & Tetraponera sp.01 & & 0.1 & & & & & & & & & & & & & & \\
\hline 80. & Tetraponera sp.02 & & & & & & & & & & & & 0.2 & & & & 0.1 \\
\hline
\end{tabular}

\section{Appendix Figure}

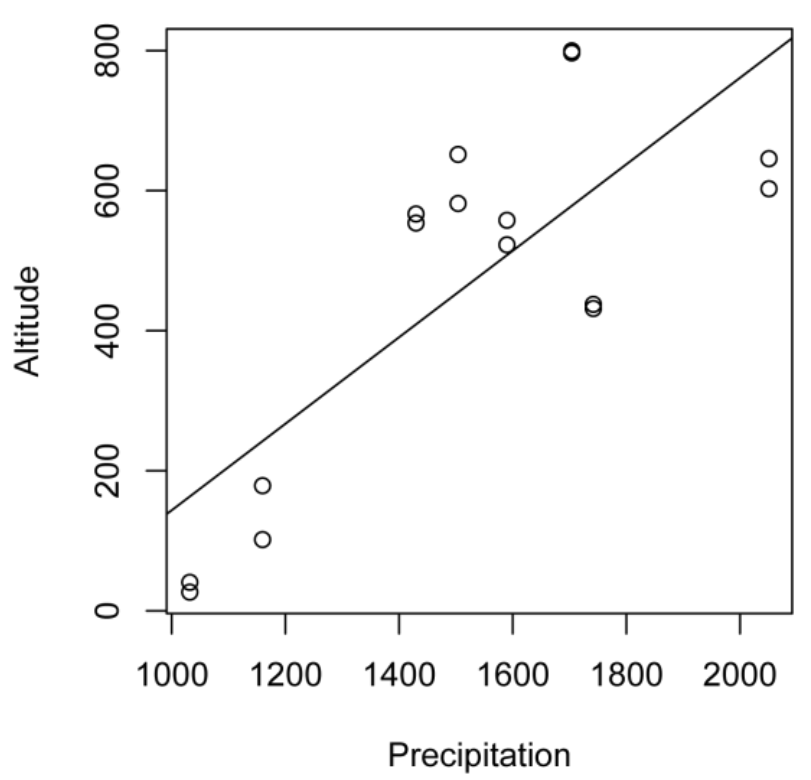

Figure A. Correlation between precipitation and altitude from 16 plots of study area $\left(\mathrm{F}_{1,14}=21.47\right.$, $\left.\mathrm{P}=0.0004, \mathrm{r}^{2}=0.58\right)$. 


\section{Chapter 3}

Long-term change of ant community structure in cacao agroforestry landscapes in Indonesia

Akhmad Rizali, Yann Clough, Damayanti Buchori, Meldy L.A. Hosang, Merijn M. Bos, Teja Tscharntke 


\section{ABSTRACT}

Land-use change and agricultural intensification can strongly affect biodiversity in agricultural landscapes. While many studies investigate management impacts, data on the long-term change of species communities in agroecosystems is scarce. We analyzed the long-term change in diversity and composition of ant communities in cacao agroforestry systems in Central Sulawesi, Indonesia and attempted to disentangle the driving factors of this change. Ant communities were resampled in 2009 from sites for which previous surveys had been conducted either in 2001 (the rainforest-poor Palolo region) or 2003 (the rainforest-rich Kulawi region) using insecticide fogging. Ant community composition changed significantly over time in Palolo and Kulawi. The change in ant species richness differed between regions. Species richness increased in Kulawi, probably due to the increasing availability of nest sites and microhabitats as trees grow larger and older. In the Palolo region, species richness decreased, suggesting the high local intensification and landscape-wide changes may have counteracted the effects of tree age. Changes in ant communities over time were significant but were more difficult to explain than expected, despite clear difference in management changes within and between regions. The findings suggest that the landscape-scale differences between the two study regions play a more important role for species diversity and its composition than changes in local management. This highlights the importance of long-term studies across contrasting landscapes for better understanding the consequences land-use intensification has on tropical biodiversity.

\section{Keywords}

Agricultural intensification, biodiversity, land-use change, pesticide application, Philidris cordata, Sulawesi 


\section{INTRODUCTION}

Habitat destruction through land-use change such as conversion of natural habitat to farmland is the most important driver of biodiversity loss in terrestrial ecosystems (Sala et al., 2000). In addition, intensification of agricultural management through reduction of crop diversity and increases in pesticide application has a negative impact on non-crop biodiversity in agricultural habitats (Geiger et al., 2010). Species surviving in such environments have to be adapted to strongly modified habitats and regular disturbance.

However, some agricultural land-use types can conserve biodiversity. Bhagwat et al. (2008) describes major reasons why agroforestry systems can be valuable for biodiversity conservation. They are important for the protection of species and habitats outside formally protected areas, because they maintain heterogeneity at local habitat and landscape scales. Furthermore, shade trees in agroforestry landscapes reduce pressure on protected forest reserves (Tscharntke et al., 2011). While low-intensity agricultural systems have been criticized for indirectly increasing pressure to convert natural habitat (Phalan et al., 2011), recent evidence suggest agroforests can maintain biodiversity within production landscapes, without reducing yields per unit area (Clough et al., 2011).

Nevertheless, as in other agroecosystems, intensification of agroforest management often negatively affects biodiversity and associated ecosystem functions. Intensification by shade tree removal in cacao agroforestry adversely affects biodiversity (Steffan-Dewenter et al., 2007) and reduces the relative abundance of predators relative to their arthropod prey (Klein et al., 2002). In addition to a decrease in complexity of vegetation structure, frequent pesticide applications accompanying intensive management may also negatively impact biodiversity, including beneficial species such as pollinating bees or predatory ants (Wiktelius et al., 1999). 
Ants occur in high abundances and with a high diversity of species in almost all natural ecosystems, including agroecosystems, with the exception of tilled arable land. Ant communities can be very species rich in tropical agroforestry systems (Philpott \& Armbrecht, 2006), but agricultural intensification, for example in coffee management by reduction in canopy complexity, causes decreases in ant species richness (Philpott et al., 2008). Thus, human disturbance and land-use change are pivotal factor affecting ant diversity and its composition (see Gibb \& Hochuli, 2003; Gómez et al., 2003). In addition, insecticide application negatively affects ant diversity, for instance in maize agroecosystems (Badji et al., 2006). Unfortunately, information about long-term patterns of ant diversity in agroecosystems, including agroforests, is lacking. This is worrying, because the species composition and the species richness of communities in transformed ecosystems may not be stable even if management does not change (Kuussaari et al., 2009). Tropical agroecosystems established after forest conversion lead to a strong erosion over time of number of forest species, but not overall species numbers, in bird communities (Daily et al., 2001; Maas et al., 2009). Understanding the value of agroforests for conservation would benefit from insights gained from the analysis of longterm datasets. For ants, this is particularly important given the major functional role they play in the agroecosystem.

Here, we studied how ant diversity changed over several years in cacao agroforestry systems in Central Sulawesi, Indonesia. Ants are very species rich in cacao, with over 160 morphospecies of ants having been recorded from these systems in a previous study (Wielgoss et al., 2010). Cacao has seen substantial intensification over the past years in Central Sulawesi, with the removal of shade trees and an increase in the frequency of pesticide applications (Wanger et al., 2010; Clough et al., 2011), but it is not known how ant communities are responding to such changes. We resampled sites where 
ants had been collected previously by Hosang (2004) and Bos (2006), with the same sampling method of previous research with fogging in the same plots and the same month. We interviewed farmers to get information about change in habitat and agricultural practices. In addition, we conducted a field experiment to test the effect of pesticide applications on the ant community. Of all management changes we expected increases in insecticide-use to have the largest impact, but at the same time farmer recollection about the use of phytochemical compounds other than herbicides was expected to be poor. We addressed the following questions: (1) What is the long-term change in richness and composition of ant communities? (2) Which factors affect the change of ant communities over time? (3) Is pesticide application the main factor affecting ant communities?

\section{METHODS}

\section{Study site}

Ants were sampled in cacao agroforests in Central Sulawesi, Indonesia, in sites previously sampled by Hosang in 2001 (Hosang, 2004) and Bos in 2003 (Bos, 2006). Plots of Hosang were located in cacao agroforestry in the Palolo valley at the eastern margin of the Lore Lindu National Park (LLNP) which is designated as part of the UNESCO World Network of Biosphere Reserves. In this research, we resampled ants in 12 of the 18 plots sampled by Hosang. Plots are distributed across three villages: Berdikari (B), Sintuwu (S) and Nopu (N) (see supporting information Fig. S1) and this cacao agroforestry landscape was classified as rainforest-poor because most plots were several kilometers away from the continuous rainforest of the national park due to large-scale deforestation from 2001 to 2009 (Erasmi et al., 2004, Erasmi unpublished data).

The plots sampled by Bos were located in cacao agroforestry systems around the village of Toro in the Kulawi valley, at the western border of LLNP. The cacao 
agroforestry landscape of Toro was classified as rainforest-rich because Toro was in 2009 still surrounded by rainforest, with only few hundred meters distance to the continuous rainforest of the national park, thereby contrasting with the Palolo region, where the main distance to the continuous forest within the national park increased between the two samplings by several kilometers due to deforestation. Bos categorized the cacao plots into three different types: agroforests shaded by forest remnants (type B), agroforests shaded by diverse stands of planted trees (type C) and agroforests shaded by one or two species of planted shade trees (type D). The conditions of all plots were almost the same when resampled in 2009.

\section{Ant sampling}

Ants were resampled in the same month as in the original study, i.e. July 2009 for Palolo (Hosang, 2004) and December 2009 for Kulawi (Bos, 2006). Ants were sampled using same method by Hosang and Bos with canopy knockdown fogging, which is an effective and widely used technique for collecting arthropods from tree crowns (Perfecto $e t$ al., 1997; Lawton et al., 1998). Both Hosang and Bos used pyrethroid insecticides (Hosang, 2004: Lambda-cyhalothrin; Bos, 2006: Permethrin). We used Malathion, an organophosphate insecticide which has a similar knock-down effect as pyrethroids (Sogorb \& Vilanova, 2002). The resampling was carried out on one randomly selected site per day between 8.00 am and $9.00 \mathrm{am}$. On each cacao tree, fogging was conducted for approximately 5 minutes or until the whole canopy was completely covered with insecticidal fog. To reduce bias due to contamination with specimens from outside the target canopy, killed ants were collected from a 4 square meter sheet of white canvas placed directly under each tree and stored in $70 \%$ alcohol for identification. 


\section{Ant identification}

Ant specimens were sorted and identified to genus level using published keys (Bolton, 1994). Ants were further sorted to units according to their external morphology (morphospecies) (Lattke, 2000). Some of the ants were identified to species level based on reliable digital resources (e.g., http://www.antweb.org). Comparing morphospecies of ants of both samples, we identified those morphospecies likely to be identical in the sampling by Hosang/Bos and our resampling (supporting information Table S1).

\section{Change in environment and agricultural management}

An interview was conducted with the owner of each cacao plot to gather data about the differences in environmental conditions and agricultural management between 2001 and 2009 in Palolo valley and between 2003 and 2009 in Kulawi valley. The focus of the interview was to gain information about habitat conditions, shade tree reduction, insecticide application, and weed management (herbicide application) at the time of the first and second sampling (detail of the questions in the supporting information Table S2). Cacao tree characteristics (age and plant size) were directly measured in terms of stem diameter and canopy height, and compared with data from Hosang and Bos.

\section{Insecticide experiment}

An insecticide experiment was conducted to test the effect of insecticide application on the ant communities in cacao. The experiment was conducted in the Palolo valley in a cacao agroforestry system where insecticides had never been used before. In total, eight plots of $8 \mathrm{~m} \times 8 \mathrm{~m}$ were used in this experiment with four plots as control and four plots as treatment (see supporting information Fig. S2). Each plot consisted of nine cacao trees 8 years of age at the start of the experiment. In the treatment plots, insecticides 
were applied every two weeks (this is the frequency of insecticide application generally used by local farmers in Palolo valley) during one year (from August 2009 to July 2010) using an insecticide with the active ingredient Chlorpirifos $200 \mathrm{~g} / \mathrm{l}$.

After a one-year insecticide treatment, in August 2010, ants were collected both in the control and treatment plots using hand collecting and baiting. On each plot, five from nine cacao trees were selected randomly for ant observation. The baiting method, following Wielgoss et al. (2010), consisted in offering tuna and sugar water on plastic observation plate. One plate was placed in the main ramification of each cacao tree and one set on the ground with 1 meter distance from each cacao tree. Ant species were observed at $15,30,45$, and 60 minute after placing the plates and the abundance of all ant species occurring at the baits was counted at each plate. At the same time, ants were also observed with hand collecting on the tree stem, in the lower canopy and on the soil surface by searching ants that were not attracted to baits. The specimens were identified to species or morphospecies as reported above.

\section{Data analysis}

Analysis of variance (ANOVA) was used to compare ant diversity between different years both in the rainforest-poor Palolo region and the rainforest-rich Kulawi region. Ant diversity was analyzed both at the tree and the plot level. Tree level analysis was conducted on the average number of species on a cacao tree for each plot. Plot level analysis was conducted on the total ant species number for each plot. For each of the two levels of analysis there was thus one value per plot per year. Species accumulation curve were used to compare ant species richness between different years in Palolo and Kulawi based on cacao tree as sampling unit. Changes in ant species composition were analyzed using non-metric multi-dimensional scaling (NMDS), based on the Bray-Curtis similarity 
index. Then, analysis of similarities (ANOSIM) was used to test for differences in ant species composition between years.

Changes of management practices such as shade-tree removal and increased pesticide application were derived from questionnaire data. We used data of shade-tree canopy cover from Hosang and Bos and compared these with current data. The differences of plant size (diameter and height) between years were analyzed using ANOVA.

The effects of environment and agricultural management on ant diversity were analyzed using linear mixed-effects model (LME). Their effects on ant community composition were analyzed using canonical correspondence analysis (CCA) with ordination and forward selection.

The effect of insecticide application on number of ant species at plot level was tested using ANOVA. We also used ANOSIM based on the Bray-Curtis similarity index, to test for differences in ant species composition between treatment and control plots. All analyses were conducted using R software (R Development Core Team, 2012).

\section{RESULTS}

\section{The long-term change of ant diversity in cacao plantation}

A total of 87 ant species belonging to five subfamilies were recorded across all samples in 2001, 2003, and 2009 from the rainforest-poor Palolo and the rainforest-rich Kulawi region (see supporting information Table S3). The change in ant species richness between the first and the second sampling period differed greatly between cacao agroforestry systems in the rainforest-poor and the rainforest-rich region (Fig. 1a, b). Based on ANOVA at the tree level, ant species richness in the rainforest-poor Palolo was significantly lower in 2009 than in 2001 (Fig. 2a; $F_{1,11}=14.180, P=0.003$ ). In contrast, in 
the rainforest-rich Kulawi, ant species richness was significantly higher in 2009 than in 2003 (Fig. 2b; $F_{1,11}=6.191, P=0.030$ ).

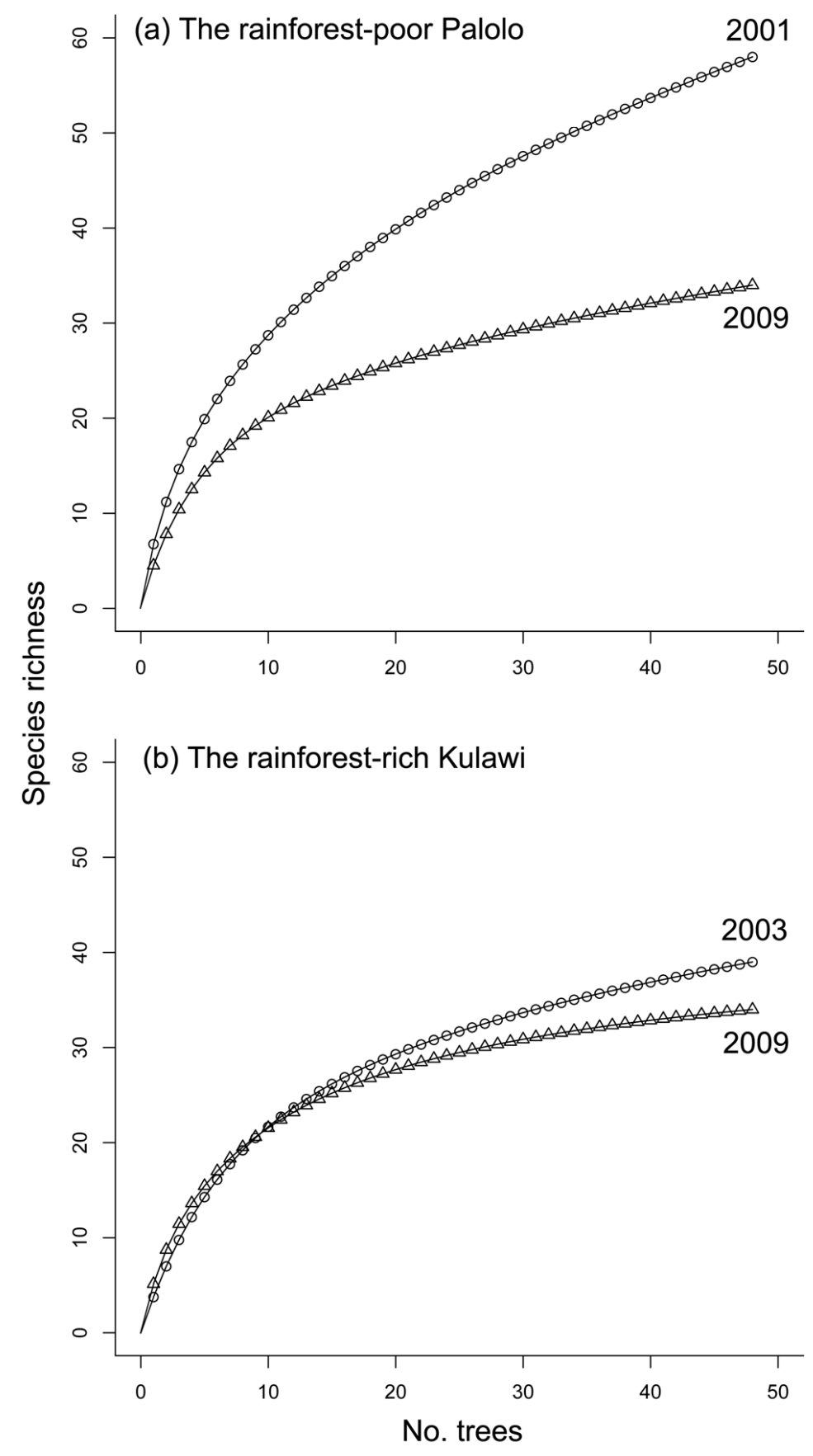

Figure 2. Species accumulation curve between different years in (a) the rainforest-poor Palolo and (b) the rainforest-rich Kulawi. 
(a)

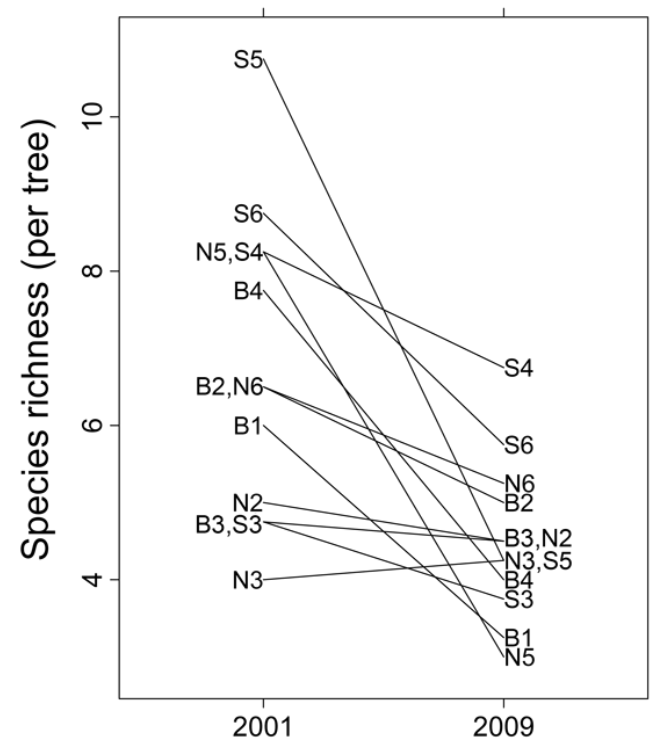

(b)

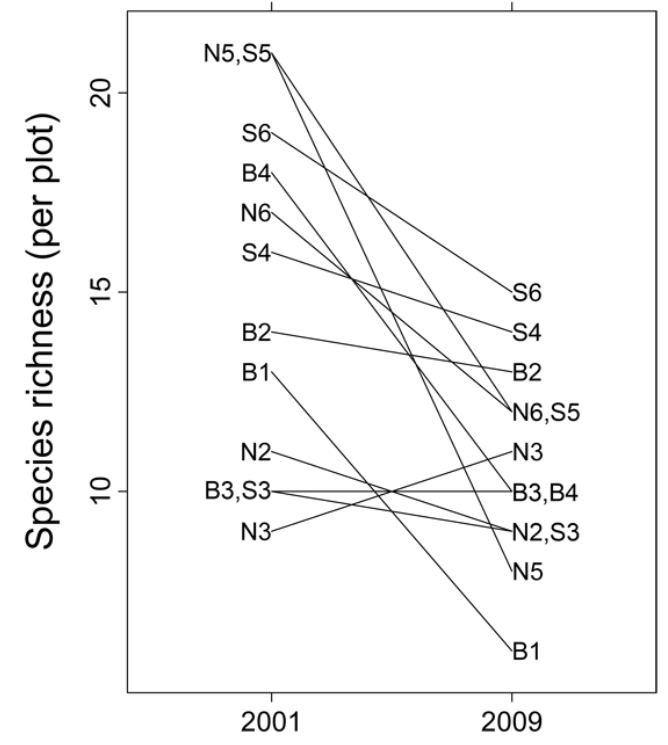

(c)

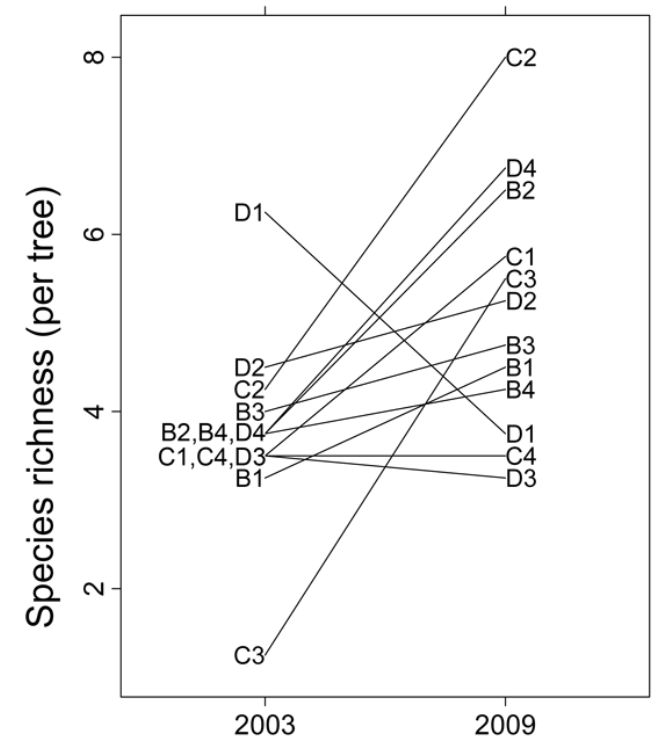

(d)

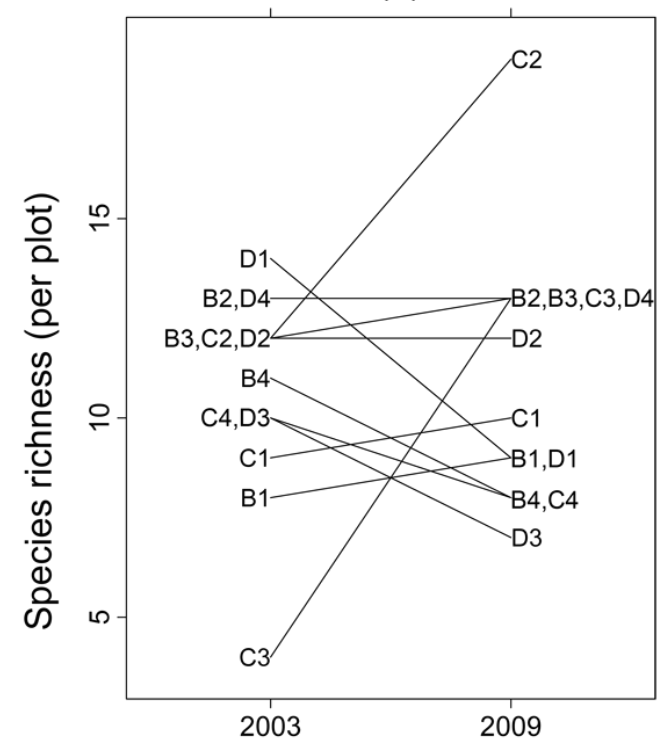

Figure 2. Change in ant species richness per plot. In the rainforest-poor Palolo region, ant richness significantly decreased both at (a) tree level (ANOVA: $F_{1,11}=14.180, P=0.003$ ) and (b) plot level (ANOVA: $F_{1,11}=10.930, P=0.007$ ). Plots are labeled with $\mathrm{N}$ : Napu village, $\mathrm{B}$ : Berdikari village, and S: Sintuwu village. In the rainforest-rich Kulawi region, ant richness significantly increased at (c) tree level (ANOVA: $\mathrm{F}_{1,11}=6.191, \mathrm{P}=0.030$ ) but not at $(\mathrm{d})$ plot level (ANOVA: $\mathrm{F}_{1,11}=0.186, \mathrm{P}$ $=0.674)$. Plots are labeled with B: agroforests shaded by forest trees, C: agroforests shaded by a diversity of planted trees, and D: agroforests shaded by one or two species of planted shade trees. 
At the plot level, ant species richness in the rainforest-poor Palolo was significantly lower in 2009 than in 2001 (Fig. 2c; $F_{1,11}=10.930, P=0.007$ ). In contrast, there was no significant overall difference of ant species richness in the rainforest-rich Kulawi between years, with diversity increasing, decreasing or remaining, depending on the plot (Fig. 2d; $\left.\mathrm{F}_{1,11}=0.186, \mathrm{P}=0.674\right)$

Based on NMDS and ANOSIM, the long-term change of ant species composition show similar patterns in the rainforest-poor Palolo region and the rainforest-rich Kulawi region, in that the ant species composition changed in Palolo (Fig. 3a, ANOSIM statistic R $=0.417, \mathrm{P}=0.001$ ) and in Kulawi (Fig. 3b, ANOSIM statistic $\mathrm{R}=0.461, \mathrm{P}=0.001$ ).

(a) The rainforest-poor Palolo

(b) The rainforest-rich Kulawi

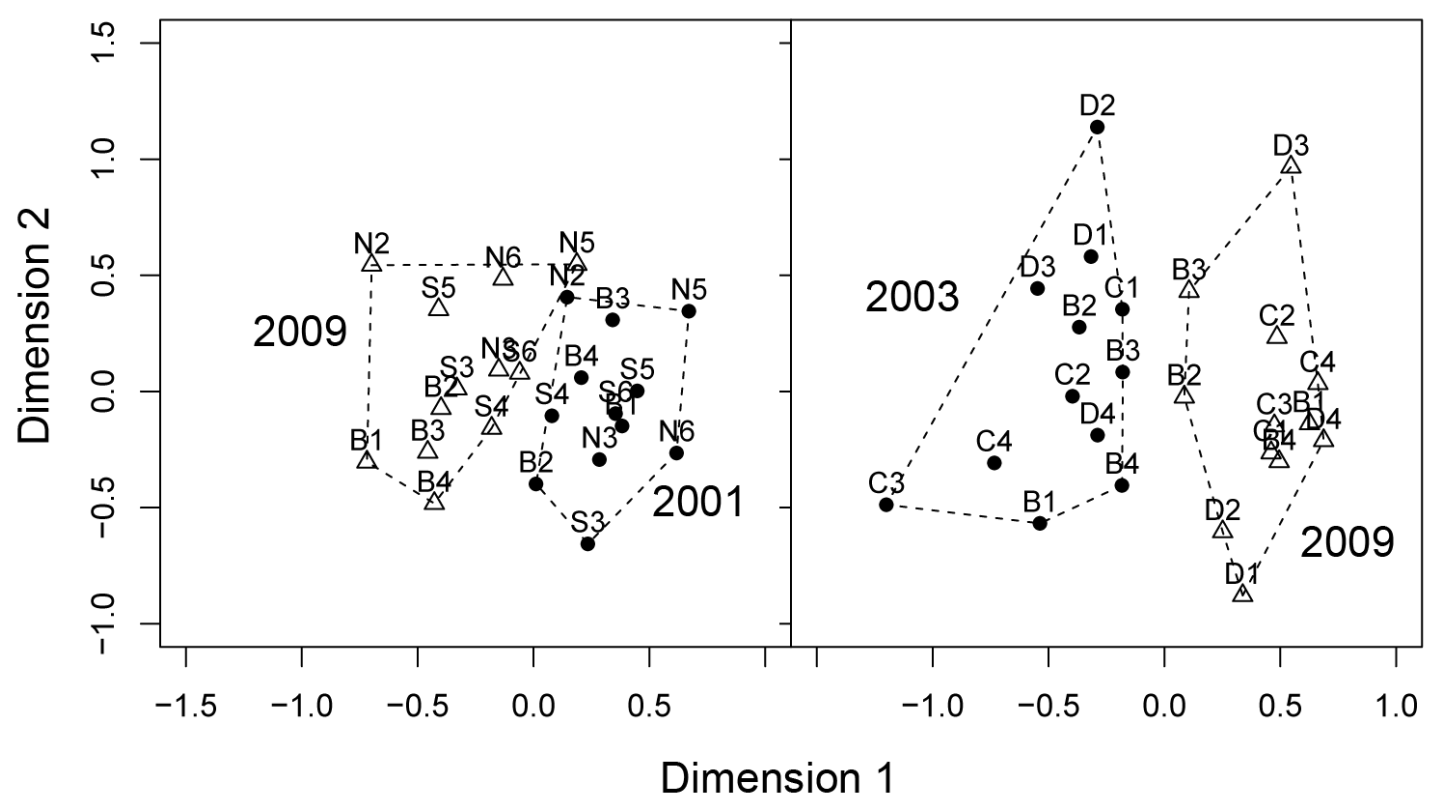

Figure 3. Two-dimensional non-metric multidimensional scaling (NMDS) of ant species community composition between plots using the Bray-Curtis dissimilarity index. (a) Ant species in the rainforest-poor Palolo in 2001 (solid circle) and 2009 (open triangle), stress $=22.46$, ANOSIM statistic $R=0.417, P=0.001$. (b) Ant species in the rainforest-rich Kulawi in 2003 (solid circle) and 2009 (open triangle), stress $=21.71$, ANOSIM statistic $\mathrm{R}=0.461, \mathrm{P}=0.001$. 
The species abundance ranking based on the number of trees occupied exhibits important differences between the first and second sampling (see Fig. 4, supporting information Fig. S3-S4). In the rainforest-poor Palolo, the most abundant ant species Philidris cordata $\left(\mathrm{F}_{1,22}=3.708, \mathrm{P}=0.067\right)$ was still very frequently encountered although the number of trees occupied tended to decrease (Fig. 4a). In the rainforest-rich Kulawi, the changes between 2003 and 2009 were considerable. P. cordata, which had not been recorded in 2003 was the most dominant ant species in $2009\left(\mathrm{~F}_{1,22}=29.146, \mathrm{P}<0.0001\right.$, Fig. 4b).

The changes in occurrence of tramp ant species (human commensal species) such as Anoplolepis gracilipes, Technomyrmex albipes, Monomorium floricola and Tapinoma melanocephalum (based on McGlynn, 1999) between years differed between species and regions (Fig. 4). In rainforest-poor Palolo, A. gracilipes and M. floricola significantly decreased (Fig. 4a). In rainforest-rich Kulawi, the tramp and invasive ant species $A$. gracilipes still occurs in cacao trees. In addition, T. albipes and M. floricola, that had not been recorded in 2003 become established in cacao in 2009 in Kulawi (Fig. 4b).

\section{Change in environment and agricultural management}

Based on the information from the farmers, agricultural management changed dramatically in rainforest-poor Palolo but only slightly in rainforest-rich Kulawi. In Palolo, age of cacao trees at the first sampling ranged from 5 to 10 years in 2001 (Hosang, 2004) and from 13 to 18 years when we resampled them. Stem diameter significantly increased (Table $\left.1 ; \mathrm{F}_{1,11}=10.34, \mathrm{P}=0.008\right)$ but the height of cacao trees did not change (Table 1; $\left.F_{1,11}=0.01, P=0.909\right)$. Canopy cover of shade trees decreased by about $44 \%$ between 2001 and 2009 (Table 1) and other changes to parts of the wider plantation, such as conversion to housing, occurred in seven out of 12 observation plots (Table 1). In Palolo, 
insecticide application frequency almost doubled between 2001 and 2009, but herbicide application frequency remained stable (Table 1).

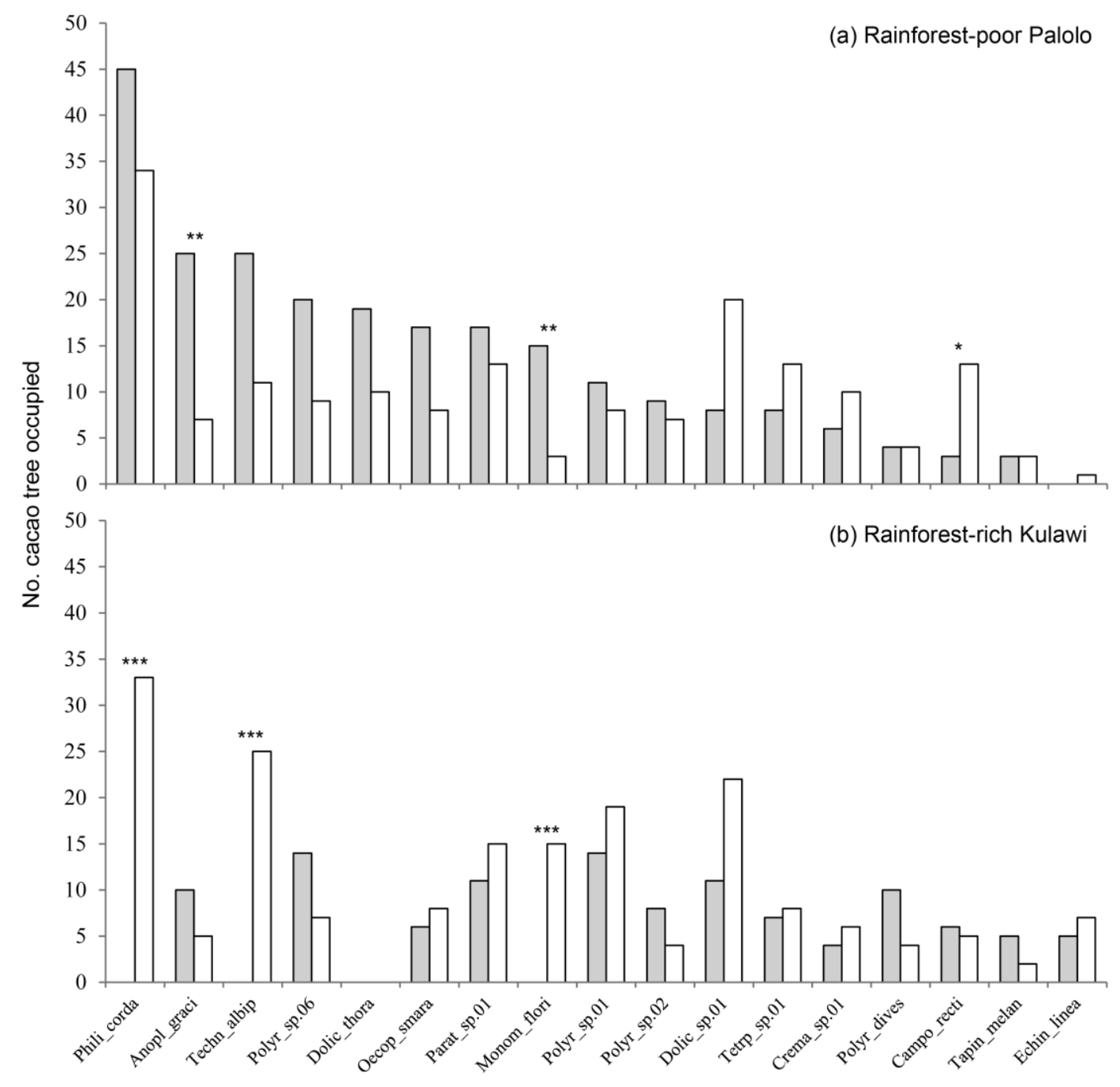

Figure 4. Changes of the highest abundance ant species based on number of trees occupied in (a) the rainforest-poor Palolo region between 2001 (solid bar) and 2009 (white bar); and (b) the rainforest-rich Kulawi region between 2003 (solid bar) and 2009 (white bar). Significance different based on ANOVA: ***: $\mathrm{P}<0.0001$, **: $\mathrm{P}<0.001$, and *: $\mathrm{P}<0.01$. Species abbreviation: Phili_corda: Philidris cordata, Anopl_graci: Anoplolepis gracilipes, Techn_albip: Technomyrmex albipes, Polyr_sp.06: Polyrachis sp.06, Dolic_thora: Dolichoderus thoracicus, Oecop_smara: Oecophylla smaragdina, Parat_sp.01: Paratrechina sp.01, Monom_flori: Monomorium floricola, Polyr_sp.01: Polyrachis sp.01, Polyr_sp.02: Polyrachis sp.02, Dolic_sp.01: Dolichoderus sp.01, Tetrp_sp.01: Tetraponera sp.01, Crema_sp.01: Crematogaster sp.01, Polyr_dives: Polyrachis dives, Campo_recti: Camponotus recticulatus, Tapin_melan: Tapinoma melanocephalum, Echin_linea: Echinopla lineata 
Table 1. Changes in tree size, habitat condition (shade trees and cacao field change), and pesticide application in the rainforest-poor Palolo from 2001 to 2009 and in the rainforest-rich Kulawi from 2003 to 2009. Data of tree size and canopy cover were derived from previous research by Hosang in 2001, Bos in 2003 and Rizali in 2009. Data of cacao field condition and pesticide application were based on information from the farmers. Data is given as means $\pm \mathrm{SE}$

\begin{tabular}{|c|c|c|c|c|c|c|}
\hline \multirow{2}{*}{ Parameters } & \multicolumn{3}{|c|}{ Rainforest-poor Palolo } & \multicolumn{3}{|c|}{ Rainforest-rich Kulawi } \\
\hline & 2001 & 2009 & Statistics & 2003 & 2009 & Statistics \\
\hline \multicolumn{7}{|l|}{ Plant size } \\
\hline Stem diameter $(\mathrm{cm})$ & $10.6 \pm 0.7$ & $12.4 \pm 0.2$ & $\begin{aligned} \mathrm{F}_{1,11} & =10.34, \\
\mathrm{P} & =0.008\end{aligned}$ & $7.9 \pm 0.5$ & $11.4 \pm 0.6$ & $\begin{array}{c}\mathrm{F}_{1,111}=48.95, \\
\mathrm{P}<0.0001\end{array}$ \\
\hline Height (cm) & $512.9 \pm 37.6$ & $509.5 \pm 12.5$ & $\begin{array}{c}\mathrm{F}_{1,11}=0.01 \\
\mathrm{P}=0.909\end{array}$ & $341.1 \pm 16.0$ & $485.7 \pm 23.7$ & $\begin{array}{c}\mathrm{F}_{1,11}=67.24, \\
\mathrm{P}<0.0001\end{array}$ \\
\hline \multicolumn{7}{|l|}{ Habitat condition } \\
\hline $\begin{array}{l}\text { Canopy cover of } \\
\text { shade trees }(\%)\end{array}$ & $60.0 \pm 5.2$ & $15.8 \pm 4.8$ & $\begin{array}{c}\mathrm{F}_{1,10}=52.64, \\
\mathrm{P}<0.0001\end{array}$ & $65.0 \pm 3.9$ & $50.8 \pm 5.1$ & $\begin{aligned} \mathrm{F}_{1,11} & =12.67, \\
\mathrm{P} & =0.004\end{aligned}$ \\
\hline $\begin{array}{l}\text { Cacao field change } \\
\text { (plot change/plot } \\
\text { total) }\end{array}$ & $0 / 12$ & $7 / 12$ & & $0 / 12$ & $0 / 12$ & \\
\hline \multicolumn{7}{|l|}{ Pesticide application } \\
\hline $\begin{array}{l}\text { Insecticide } \\
\text { (time/year) }\end{array}$ & $6.9 \pm 2.2$ & $12 \pm 2.9$ & $\begin{array}{c}\mathrm{F}_{1,11}=4.55 \\
\mathrm{P}=0.058\end{array}$ & 0 & $1.7 \pm 0.9$ & $\begin{array}{c}\mathrm{F}_{1,11}=3.57 \\
\mathrm{P}=0.085\end{array}$ \\
\hline $\begin{array}{l}\text { Herbicide } \\
\text { (time/year) }\end{array}$ & $2.4 \pm 1.1$ & $1.6 \pm 0.4$ & $\begin{array}{c}\mathrm{F}_{1,11}=0.39 \\
\mathrm{P}=0.553\end{array}$ & $0.1 \pm 0.1$ & $1.5 \pm 0.6$ & $\begin{array}{c}\mathrm{F}_{1,11}=5.20 \\
\mathrm{P}=0.043\end{array}$ \\
\hline
\end{tabular}

In the rainforest-rich Kulawi, the age of cacao trees ranged from 7 to 10 years in 2003 (Bos, 2006), and from 13 to 16 years at the time of resampling in 2009. The stem diameter significantly increased (Table $\left.1 ; \mathrm{F}_{1,11}=48.95, \mathrm{P}<0.0001\right)$ and the height of cacao trees also significantly increased (Table $1 ; \mathrm{F}_{1,11}=67.24, \mathrm{P}<0.0001$ ). Shade tree cover slightly decreased by about $14 \%$ (Table 1) and conversion of parts of the plantation to other uses did not occur. The farmers in Kulawi increased insecticide and herbicide use (Table 1). Herbicides were increasingly used to control weeds, especially in plots shaded by only one or two species of planted shade trees (type D), with a slightly higher frequency in 2009 than in 2003. 


\section{Effects of agricultural management and environmental change on ant diversity}

Based on the linear mixed effect models (LME), changes in insecticides and herbicides were not significantly related to ant diversity change in the rainforest-poor Palolo (Table 2), although there was a non-significant trend for a negative effect of insecticide applications on ant species richness The changes in shade tree cover and age of the cacao trees were not included in the LME analysis due to their high correlation with year (shade trees, Pearson's correlation: $r=-0.79$; age: $r=0.95$ ). Insecticide and herbicide applications were also not related to ant diversity change in Kulawi (Table 2).

Table 2. Linear mixed-effects models run using restricted maximum likelihood with number of species as the response variable, insecticide and herbicide as fixed effects. Random intercepts were included for each plot. Estimates are given for the intercept and the slopes for the fixed effects.

\begin{tabular}{|lrcccc|}
\hline \multicolumn{7}{|c|}{ The rainforest-poor PALOLO } \\
\hline Fixed effect & Estimate & Std.Error & DF & $\mathrm{t}$-value & $\mathrm{p}$-value \\
intercept & 13.955 & 1.140 & 11 & 12.240 & 0.000 \\
insecticide & -0.170 & 0.090 & 10 & -1.810 & 0.100 \\
herbicide & 0.266 & 0.342 & 10 & 0.777 & 0.455 \\
\hline \multicolumn{7}{|c|}{ The rainforest-rich KULAWI } \\
\hline Fixed effect & Estimate & Std.Error & DF & t-value & p-value \\
intercept & 10.433 & 0.701 & 11 & 14.877 & 0.000 \\
insecticide & 0.059 & 0.343 & 10 & 0.171 & 0.868 \\
herbicide & 0.549 & 0.481 & 10 & 1.141 & 0.280 \\
\hline
\end{tabular}

The CCA revealed a marginally non-significant relationship between insecticide application and ant species composition both in Palolo $(\mathrm{P}=0.076)$ and Kulawi $(\mathrm{P}=0.075)$ (Table 3). Ordination with CCA also revealed that insecticide was an important variable affecting ant species composition in cacao agroforestry in Palolo (Figure 5) and Kulawi (Figure 6). 
Table 3. Effects of explanatory variables related to environment and agricultural management on ant species composition in the rainforest-poor Palolo and the rainforest-rich Kulawi: results of a forward selection procedure within a canonical correspondence analysis (CCA) using ordistep method with 1000 permutations. Significant codes: $0.01=* *, 0.05=*$

\begin{tabular}{|lccccl|}
\hline PALOLO & & & & & \\
\hline Parameter & df & AIC & \multicolumn{1}{l|}{ F } & N.Perm & $\operatorname{Pr}(>\mathrm{F})$ \\
Age & 1 & 230.05 & 1.909 & 999 & 0.335 \\
Diameter & 1 & 226.49 & 5.731 & 999 & $0.018^{*}$ \\
Habitat & 1 & 230.79 & 1.182 & 999 & 0.515 \\
Herbicide & 1 & 230.77 & 1.202 & 999 & 0.396 \\
High & 1 & 231.18 & 0.814 & 999 & 0.666 \\
Insecticide & 1 & 228.30 & 3.718 & 999 & 0.076 \\
Shade & 1 & 230.73 & 1.243 & 999 & 0.514 \\
Year & 1 & 230.06 & 1.898 & 999 & 0.340 \\
\hline KULAWI & & & & & \\
\hline Parameter & df & AIC & F & N.Perm & $\operatorname{Pr}(>\mathrm{F})$ \\
Age & 1 & 222.57 & 1.147 & 999 & 0.115 \\
Diameter & 1 & 223.42 & 0.345 & 999 & 0.717 \\
Habitat & 0 & 221.79 & 0 & 0 & \\
Herbicide & 1 & 223.19 & 0.554 & 999 & 0.537 \\
High & 1 & 223.03 & 0.712 & 999 & 0.275 \\
Insecticide & 1 & 221.86 & 1.842 & 999 & 0.075 \\
Shade & 1 & 222.73 & 0.995 & 999 & 0.163 \\
Year & 1 & 221.20 & 2.505 & 999 & $0.004^{* *}$ \\
\hline
\end{tabular}

\section{Insecticide experiment}

In the insecticide experiment, 17 ant species were recorded both from control and treatment plots (Table D). A. gracilipes was the most abundant species and found in all control and treatment plots. Insecticide application does not affect ant diversity which not significantly differed between control and treatment plots $\left(\mathrm{F}_{1,6}=2.46 ; \mathrm{P}=0.168\right)$. Based on ANOSIM, insecticide application affects the changes ant species composition between control and treatment plots (ANOSIM statistic $\mathrm{R}=0.10, \mathrm{P}=0.008$ ). 


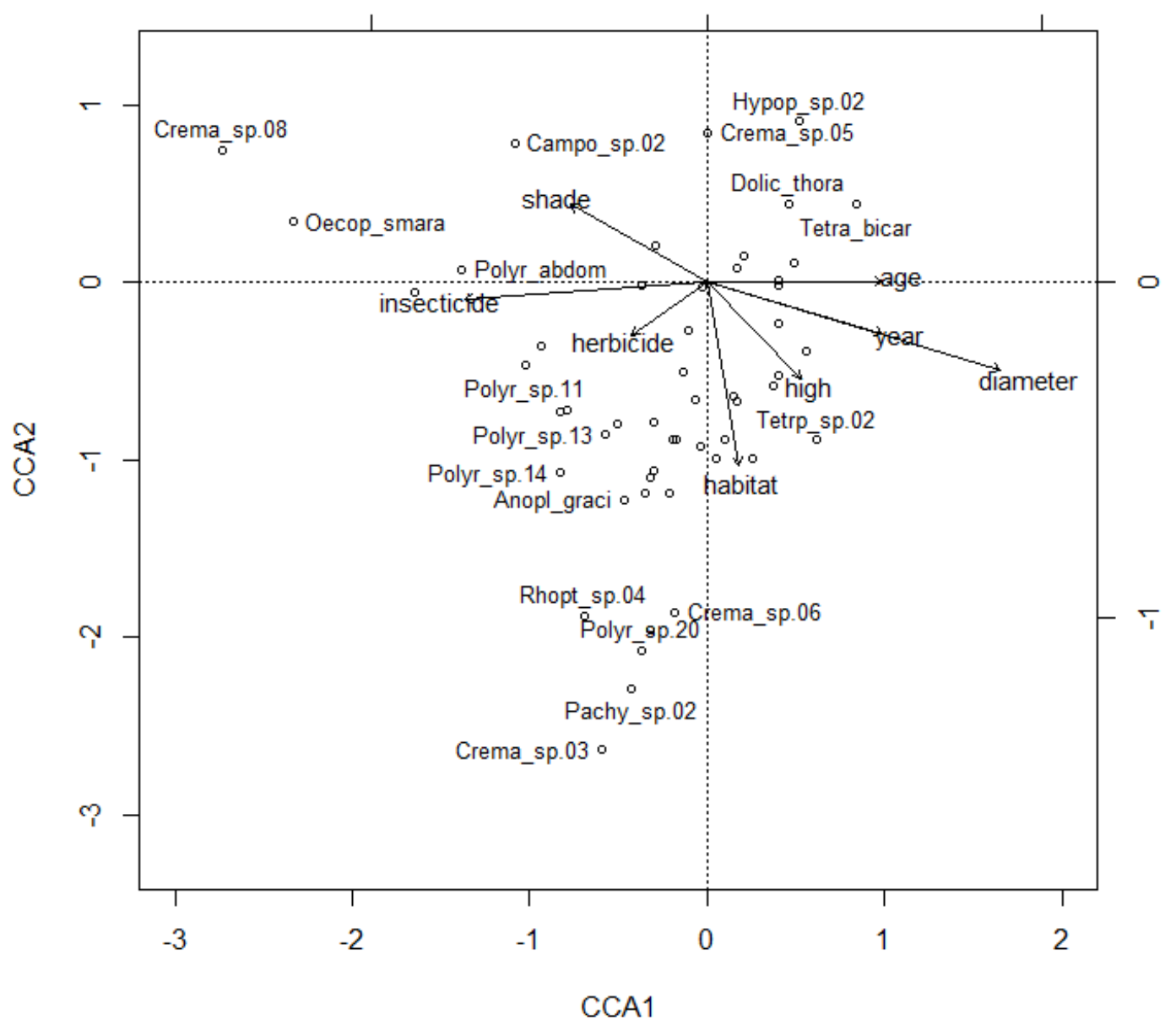

Figure 5. Canonical correspondence analysis (CCA) ordination for ant species abundance data in the rainforest-poor Palolo. Arrows indicate environmental variables and its lengths indicate the relative importance and the directions of which are obtained from the correlation of the variable to the axes. The orthogonal projection of a species point onto an environmental arrow represents the approximate center of the species distribution along that particular environmental gradient. Species are indicated with circle point and labeled with first 5 letters of their genus and species or morphospecies name respectively. Some species are not labeled in order to avoid congested graph. 


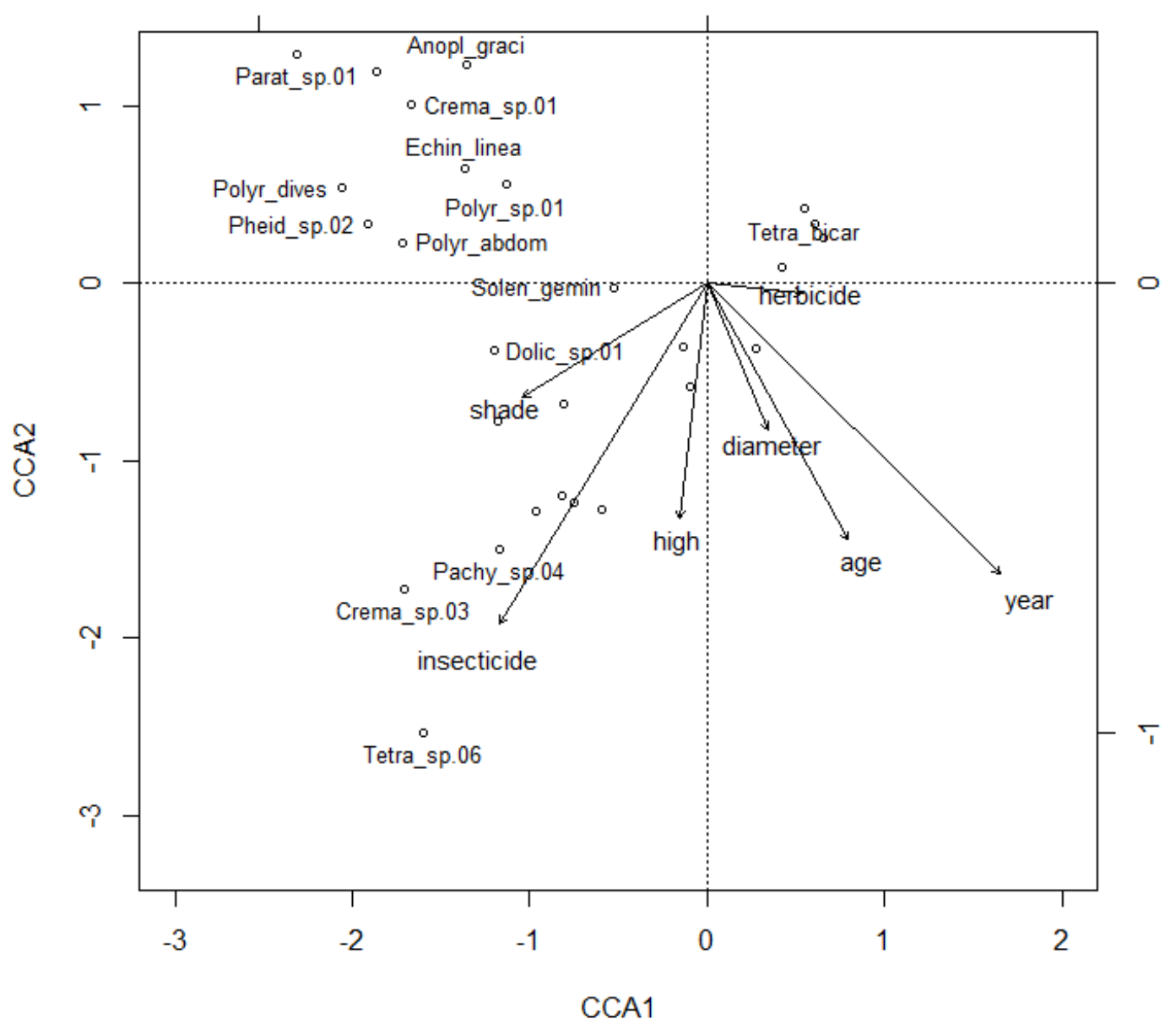

Figure 6. Canonical correspondence analysis (CCA) ordination for ant species abundance data in the rainforest-rich Kulawi. Arrows indicate environmental variables and its lengths indicate the relative importance and the directions of which are obtained from the correlation of the variable to the axes. The orthogonal projection of a species point onto an environmental arrow represents the approximate center of the species distribution along that particular environmental gradient. Species are indicated with circle point and labeled with first 5 letters of their genus and species or morphospecies name respectively. Some species are not labeled in order to avoid congested graph. 


\section{DISCUSSION}

Resampling ant communities of cacao agroforestry systems after six to eight years revealed important changes both in terms of species composition and in terms of species richness. Overall, land-use was intensified, albeit not consistently within and between regions. While changes in community structure over time are common and based on both natural temporal turnover (Greenslade, 2008) and changes due to increasing microhabitat availability as trees age (Dejean et al., 2008), we expected that agricultural intensification would be a major driver of ant community change.

In our study, the results depended both on the region and on the scale at which diversity measures were calculated, i.e. tree-level or plot-level ant communities. Plot-level and tree-level species richness decreased strongly in the rainforest-poor Palolo region. In contrast, plot-level richness did not decrease, and ant richness per tree increased in the rainforest-rich Kulawi region. Increasing tree size and age may increase nesting site availability and microhabitat structure, a primary factor for ant occurrence (Andersen, 2000). The diversity increase in Kulawi was likely related to the growth of the cacao trees, associated with increases in structural complexity of cacao trees and abundance of pruning scars and cracks in the bark. In the Palolo region, regional characteristics such as high local intensification and landscape-wide intensification of agriculture and deforestation may have counteracted the positive effect of tree age which we detected in Kulawi. In this case, ant diversity and its composition in Palolo may have been strongly influenced by anthropogenic disturbance and land-use change, as was shown for other regions of Asia (Narendra et al., 2011). Although cacao is the dominant land-use besides paddy rice in both regions, landscape-scale cacao management and land-use change differed strongly. Palolo was characterized by high management intensification with decreasing canopy cover of shade trees and an increase in the frequency of pesticide applications. In contrast, 
number of shade trees has decreased only slightly in Kulawi, and pesticide use remained infrequent. On a landscape scale, deforestation has progressed much more rapidly in Palolo than in Kulawi since 1999 (Erasmi et al., 2004; Erasmi unpublished data).

Previous studies suggested that a decrease in shade tree cover can play a major role in reducing the number of forest species, thereby changing the composition but not the species richness, of ant communities (Bos et al., 2007). In addition to shade trees, insecticide applications can also play an important role. While in some agroecosystems ant communities were found to be little affected by insecticide applications (Matlock \& de la Cruz, 2003; Chong et al., 2007), other studies found insecticides to decrease ant diversity (Badji et al., 2006) and disturb trophic interaction between ants and phytophagous insects (Perfecto, 1990; Kenne et al., 2003).

In order to shed more light on the drivers of change in ant communities in our study, we collected information on changes in plot-level management by interviewing farmers and correlated this data with the changes in ant community diversity. The change in canopy cover of shade trees within regions was closely correlated with year, but it is unlikely that reduction of shade level was responsible for the observed changes, given that the absolute change in species richness with shade tree cover reported in previous studies was not significant (Bos et al., 2007; Wielgoss et al., 2010). While herbicide use had no effect on ant species richness in either region, there was a weak trend towards a negative effect of insecticide on ant species richness in Palolo, the region in which average insecticide application frequency was highest.

It was apparent during the interviews that farmers did not have perfect recollection of past conditions and practices, and could only estimate roughly the change in agricultural practices. Therefore, in order to supplement the questionnaire data, we conducted a yearlong insecticide experiment and recorded the ant communities. Surprisingly, we also found 
only a marginally non-significant trend in species richness. However, we found that insecticide application affects ant community composition in cacao agroforestry. Several ant species sustained population declines with insecticide application and then disappeared in long-term.

Given their simplified structure compared to natural forest habitat, agroforestry systems can be vulnerable to ant invasions (Greenslade, 2008), which, in turn may lead to decreases in ant species richness as local ant colonies become outcompeted. Five tramp ants were recorded both during the original survey and the resampling. Surprisingly, in Kulawi, the increase in plot-level ant species richness was accompanied by the appearance of the invasive ant $P$. cordata in almost $(75 \%)$ all plots. This was unexpected since in a previous study the presence of $P$. cordata was found to be negatively related to ant species richness (Wielgoss et al., 2010). Interestingly, the invasive species A. gracilipes, which was a numerically dominant species in Kulawi during the original survey (Bos et al., 2008), was detected much less frequently during the second survey.

\section{CONCLUSIONS}

In conclusion, the change in ant communities in managed systems over time can be more difficult to predict than expected, highlighting the importance of repeating such longterm studies to improve our understanding of the processes underlying patterns. We found that ant species richness in cacao agroforestry may either decrease or increase significantly with time, with differences in plot-level management such as insecticide application frequency explaining only a small part of this change. The large differences in landscapescale changes between the two regions studied (high vs. low land-use intensification and deforestation levels) and the corresponding differences in change in ant communities suggest that plot and tree-level species richness depends on changes at larger scales. 
Therefore, findings from other agroecosystems that complex landscapes can compensate for local agricultural intensification (Schmidt et al., 2005; Tscharntke et al., 2005) may also apply here. Interestingly, this may suggest that supporting biodiversity-friendly management by individual smallholders, through biodiversity-friendly certification for example, may not yield substantial benefits in terms of ant diversity unless the wider landscape is also targeted. The relative role of local and landscape-level land-use patterns will need to be formally tested as more datasets become available.

\section{ACKNOWLEDGEMENTS}

We are grateful to Adha Sari and Putri Syahierah for support during laboratory research in Bogor Agricultural University (IPB). Thanks to PT. Sucofindo (Palu Branch Office) cq. Bpk. Nyoman who gave permission to use the fogging machine. Also thanks to our field assistants (Fifin, Ahmad, Boni, and Baswan) and laboratory assistants (Nur Ivon and Dewi Utaminingsih). Thanks to the Center for Tropical Forest Margin (CTFM), University of Tadulako for supporting the administration and transportation during field survey. This research project was funded by the German Science Foundation (DFG), the University of Göttingen, Bogor Agricultural University, and Directorate General of Higher Education, Ministry of National Education - the Republic of Indonesia.

\section{REFERENCES}

Andersen, A.N. (2000) Global ecology of rainforest ants: functional groups in relation to environmental stress and disturbance. Ants: Standard Methods for Measuring and Monitoring Biodiversity (ed. by D. Agosti, J.D. Majer, L.E. Alonso and T.R. Schultz), pp. 25-34. Smithsonian Institution Press, Washington. 
Badji, C.A., Guedes, R.N.C., Corrêa, A.S., Ferreira, G.H. \& Nascimento, I.C. (2006) Deltamethrin-induced impact on ant assemblages in tropical maize fields under conventional and no-tillage cultivation. Sociobiology, 48, 701-715.

Bhagwat, S.A., Willis, K.J., Birks, H.J.B. \& Whittaker, R.J. (2008) Agroforestry: a refuge for tropical biodiversity? Trends in Ecology \& Evolution, 23, 261-267.

Bolton, B. (1994) Identification Guide to the Ant Genera of the World. Harvard University Press, Cambridge.

Bos, M.M. (2006) Insect diversity and trophic interactions in shaded cacao agroforestry and natural forests in Indonesia. PhD Thesis, University of Göttingen, Göttingen.

Bos, M.M., Steffan-Dewenter, I. \& Tscharntke, T. (2007) The contribution of cacao agroforests to the conservation of lower canopy ant and beetle diversity in Indonesia. Biodiversity and Conservation, 16, 2429-2444.

Bos, M.M., Tylianakis, J.M., Steffan-Dewenter, I. \& Tscharntke, T. (2008) The invasive yellow crazy ant and the decline of forest ant diversity in Indonesian cacao agroforests. Biological Invasions, 10, 1399-1409.

Chong, C.S., Hoffmann, A.A. \& Thomson, L.J. (2007) Commercial agrochemical applications in vineyards do not influence ant communities. Environmental Entomology, 36, 1374-1383.

Clough, Y., Barkmann, J., Juhrbandt, J., Kessler, M., Wanger, T.C., Anshary, A., Buchori, D., Cicuzza, D., Darras, K., Putra, D.D., Erasmi, S., Pitopang, R., Schmidt, C., Schulze, C.H., Seidel, D., Steffan-Dewenter, I., Stenchly, K., Vidal, S., Weist, M., Wielgoss, A.C. \& Tscharntke, T. (2011) Combining high biodiversity with high yields in tropical agroforests. Proceedings of the National Academy of Sciences, USA, 108, 8311-8316. 
Daily, G.C., Ehrlich, P.R. \& Sánchez-Azofeifa, G.A. (2001) Countryside biogeography: use of human-dominated habitats by the avifauna of Southern Costa Rica. Ecological Applications, 11, 1-13.

Dejean, A., Djiéto-Lordon, C., Céréghino, R. \& Leponce, M. (2008) Ontogenetic succession and the ant mosaic: An empirical approach using pioneer trees. Basic and Applied Ecology, 9, 316-323.

Erasmi, S., Twele, A., Ardiansyah, M., Malik, A. \& Kappas, M. (2004) Mapping deforestation and land-cover conversion at the rainforest margin in Central Sulawesi, Indonesia. EARSeL eProceedings, 3, 388-397.

Geiger, F., de Snoo, G.R., Berendse, F., Guerrero, I., Morales, M.B., Oñate, J.J., Eggers, S., Pärt, T., Bommarco, R., Bengtsson, J., Clement, L.W., Weisser, W.W., Olszewski, A., Ceryngier, P., Hawro, V., Inchausti, P., Fischer, C., Flohre, A., Thies, C. \& Tscharntke, T. (2010) Landscape composition influences farm management effects on farmland birds in winter: A pan-European approach. Agriculture, Ecosystems \& Environment, 139, 571-577.

Gibb, H. \& Hochuli, D.F. (2003) Colonisation by a dominant ant facilitated by anthropogenic disturbance: effects on ant assemblage composition, biomass and resource use. Oikos, 103, 469-478.

Gómez, C., Casellas, D., Oliveras, J. \& Bas, J.M. (2003) Structure of ground-foraging ant assemblages in relation to land-use change in the northwestern Mediterranean region. Biodiversity and Conservation, 12, 2135-2146.

Greenslade, P. (2008) Climate variability, biological control and an insect pest outbreak on Australia's Coral Sea islets: lessons for invertebrate conservation. Journal of Insect Conservation, 12, 333-342. 
Hosang, M.L.A. (2004) Interactions between Natural Enemies, Herbivores and Cacao in Palolo Valley, Central Sulawesi. PhD Thesis, Bogor Agricultural University, Bogor.

Kenne, M., Djiéto-Lordon, C., Orivel, J., Mony, R., Fabre, A. \& Dejean, A. (2003) Influence of insecticide treatments on Ant-Hemiptera associations in tropical plantations. Journal of Economic Entomology, 96, 251-258.

Klein, A.-M., Steffan-Dewenter, I. \& Tscharntke, T. (2002) Predator-prey ratios on cocoa along a land-use gradient in Indonesia. Biodiversity and Conservation, 11, 683-693.

Kuussaari, M., Bommarco, R., Heikkinen, R.K., Helm, A., Krauss, J., Lindborg, R., Ockinger, E., Partel, M., Pino, J., Roda, F., Stefanescu, C., Teder, T., Zobel, M. \& Steffan-Dewenter, I. (2009) Extinction debt: a challenge for biodiversity conservation. Trends in Ecology \& Evolution, 24, 564-571.

Lattke, J.E. (2000) Specimen processing: building and curating an ant collection. Ants: Standard Methods for Measuring and Monitoring Biodiversity (ed. by D. Agosti, J.D. Majer, L.E. Alonso and T.R. Schultz), pp. 155-171. Smithsonian Institution Press, Washington.

Lawton, J.H., Bignell, D.E., Bolton, B., Bloemers, G.F., Eggleton, P., Hammond, P.M., Hodda, M., Holt, R.D., Larsen, T.B., Mawsley, N.A., Stork, N.E., Srivastava, D.S. \& Watt, A.D. (1998) Biodiversity inventories, indicator taxa and effects of habitat modification in tropical forest. Nature, 391, 72-76.

Maas, B., Putra, D.D., Waltert, M., Clough, Y., Tscharntke, T. \& Schulze, C.H. (2009) Six years of habitat modification in a tropical rainforest margin of Indonesia do not affect bird diversity but endemic forest species. Biological Conservation, 142, $2665-2671$. 
Matlock, R.B. \& de la Cruz, R. (2003) Ants as indicators of pesticide impacts in banana. Environmental Entomology, 32, 816-829.

McGlynn, T.P. (1999) The worldwide transfer of ants: geographical distribution and ecological invasions. Journal of Biogeography, 26, 535-548.

Narendra, A., Gibb, H. \& Ali, T.M. (2011) Structure of ant assemblages in Western Ghats, India: role of habitat, disturbance and introduced species. Insect Conservation and Diversity, 4, 132-141.

Perfecto, I. (1990) Indirect and direct effects in a tropical agroecosystem: the maize-pestant system in Nicaragua. Ecology, 71, 2125-2134.

Perfecto, I., Vandermeer, J., Hanson, P. \& Cartín, V. (1997) Arthropod biodiversity loss and the transformation of a tropical agro-ecosystem. Biodiversity and Conservation, 6, 935-945.

Phalan, B., Balmford, A., Green, R.E. \& Scharlemann, J.P.W. (2011) Minimising the harm to biodiversity of producing more food globally. Food Policy, 36, S62-S71.

Philpott, S.M. \& Armbrecht, I. (2006) Biodiversity in tropical agroforests and the ecological role of ants and ant diversity in predatory function. Ecological Entomology, 31, 369-377.

Philpott, S.M., Arendt, W.J., Armbrecht, I., Bichier, P., Diestch, T.V., Gordon, C., Greenberg, R., Perfecto, I., Reynoso-Santos, R., Soto-Pinto, L., Tejeda-Cruz, C., Williams-Linera, G., Valenzuela, J. \& Zolotoff, J.M. (2008) Biodiversity loss in Latin American coffee landscapes: review of the evidence on ants, birds, and trees. Conservation Biology, 22, 1093-1105.

R Development Core Team (2012) R: A language and environment for statistical computing. R Foundation for Statistical Computing. 
Sala, O.E., Chapin, F.S., Armesto, J.J., Berlow, E., Bloomfield, J., Dirzo, R., HuberSanwald, E., Huenneke, L.F., Jackson, R.B., Kinzig, A., Leemans, R., Lodge, D.M., Mooney, H.A., Oesterheld, M., Poff, N.L., Sykes, M.T., Walker, B.H., Walker, M. \& Wall, D.H. (2000) Biodiversity - Global biodiversity scenarios for the year 2100. Science, 287, 1770-1774.

Schmidt, M.H., Roschewitz, I., Thies, C. \& Tscharntke, T. (2005) Differential Effects of Landscape and Management on Diversity and Density of Ground-Dwelling Farmland Spiders. Journal of Applied Ecology, 42, 281-287.

Sogorb, M.A. \& Vilanova, E. (2002) Enzymes involved in the detoxification of organophosphorus, carbamate and pyrethroid insecticides through hydrolysis. Toxicology Letters, 128, 215-228.

Steffan-Dewenter, I., Kessler, M., Barkmann, J., Bos, M.M., Buchori, D., Erasmi, S., Faust, H., Gerold, G., Glenk, K., Gradstein, S.R., Guhardja, E., Harteveld, M., Hertel, D., Hohn, P., Kappas, M., Kohler, S., Leuschner, C., Maertens, M., Marggraf, R., Migge-Kleian, S., Mogea, J., Pitopang, R., Schaefer, M., Schwarze, S., Sporn, S.G., Steingrebe, A., Tjitrosoedirdjo, S.S., Tjitrosoemito, S., Twele, A., Weber, R., Woltmann, L., Zeller, M. \& Tscharntke, T. (2007) Tradeoffs between income, biodiversity, and ecosystem functioning during tropical rainforest conversion and agroforestry intensification. Proceedings of the National Academy of Sciences, USA, 104, 4973-4978.

Tscharntke, T., Klein, A.M., Kruess, A., Steffan-Dewenter, I. \& Thies, C. (2005) Landscape perspectives on agricultural intensification and biodiversity - ecosystem service management. Ecology Letters, 8, 857-874.

Tscharntke, T., Clough, Y., Bhagwat, S.A., Buchori, D., Faust, H., Hertel, D., Holscher, D., Juhrbandt, J., Kessler, M., Perfecto, I., Scherber, C., Schroth, G., Veldkamp, E. 
\& Wanger, T.C. (2011) Multifunctional shade-tree management in tropical agroforestry landscapes - a review. Journal of Applied Ecology, 48, 619-629.

Wanger, T.C., Rauf, A. \& Schwarze, S. (2010) Pesticides and tropical biodiversity. Frontiers in Ecology and the Environment, 8, 178-179.

Wielgoss, A., Tscharntke, T., Buchori, D., Fiala, B. \& Clough, Y. (2010) Temperature and a dominant dolichoderine ant species affect ant diversity in Indonesian cacao plantations. Agriculture, Ecosystems \& Environment, 135, 253-259.

Wiktelius, S., Chiverton, P.A., Meguenni, H., Bennaceur, M., Ghezal, F., Umeh, E.-D.N., Egwuatu, R.I., Minja, E., Makusi, R., Tukahirwa, E., Tinzaara, W. \& Deedat, Y. (1999) Effects of insecticides on non-target organisms in African agroecosystems: a case for establishing regional testing programmes. Agriculture, Ecosystems \& Environment, 75, 121-131. 
Chapter 3 - Long-term change of ant community structure

\section{SUPPORTING INFORMATION}

\section{Appendix Tables}

Table S1. Comparing morphospecies of ant from Hosang (2004) and Bos (2006) with author specimens.

\begin{tabular}{|c|c|c|}
\hline Previous morphospecies & Researcher & $\begin{array}{l}\text { Similar species or morphospecies } \\
\text { with author }\end{array}$ \\
\hline Formicidae sp.\#36 and Formicidae sp.01 & Hosang & Dolichoderus sp.01 \\
\hline $\begin{array}{l}\text { Bothriomyrmex sp.\#0?(Queen), Dolichoderinae } \\
\text { sp.\#1(Queen), Dolichoderinae sp.\#3, Formicidae } \\
\text { sp.\#8, Iridomyrmex sp.\#1, Iridomyrmex sp.\#2, and } \\
\text { Iridomyrmex sp.\#4 }\end{array}$ & Hosang & Philidris cordata \\
\hline Tapinoma sp.01 & $\begin{array}{l}\text { Hosang \& } \\
\text { Bos }\end{array}$ & Tapinoma melanocephalum \\
\hline Tapinoma sp.03 & Hosang & Tapinoma sp.01 \\
\hline Technomyrmex sp.01 & Hosang & Technomyrmex albipes \\
\hline Technomyrmex sp.02 & Hosang & Technomyrmex sp.01 \\
\hline Technomyrmex sp.05 & Hosang & Technomyrmex sp.02 \\
\hline Anoplolepis longipes & Hosang & Anoplolepis gracilipes \\
\hline Bothriomyrmex sp.01 & $\begin{array}{l}\text { Hosang \& } \\
\text { Bos }\end{array}$ & Camponotus recticulatus \\
\hline Echinopla sp.01 & Bos & Echinopla lineata \\
\hline Iridomyrmex sp.\#5 and Linepithema sp.\#1 & Hosang & Paratrechina sp.03 \\
\hline Polyrhachis sp.03 & Bos & Polyrhachis abdominalis \\
\hline Polyrhachis sp.07 & Bos & Polyrhachis dives \\
\hline Polyrhachis sp.03 & Hosang & Polyrhachis dives \\
\hline Polyrhachis sp.05 & Bos & Polyrhachis sp.01 \\
\hline Polyrhachis sp.07 & Hosang & Polyrhachis sp.01 \\
\hline Polyrhachis sp.11 & Bos & Polyrhachis sp.02 \\
\hline Polyrhachis sp.08 & Hosang & Polyrhachis sp.02 \\
\hline Polyrhachis sp.10 & Bos & Polyrhachis sp.04 \\
\hline Polyrhachis sp.13 & Bos & Polyrhachis sp.06 \\
\hline Polyrhachis sp.04 & Hosang & Polyrhachis sp.06 \\
\hline Polyrhachis sp.06 & Hosang & Polyrhachis sp.07 \\
\hline Polyrhachis sp.09 & Hosang & Polyrhachis sp.08 \\
\hline Crematogaster sp.07 & Hosang & Crematogaster sp.01 \\
\hline Monomorium sp.06 & Hosang & Monomorium floricola \\
\hline Monomorium sp.04 & Hosang & Monomorium sp.01 \\
\hline Tetramorium sp.08 & Hosang & Tetramorium pasificum \\
\hline Tetramorium sp.01 & Hosang & Tetramorium smithi \\
\hline Tetramorium sp.03 & Bos & Tetramorium sp.06 \\
\hline Tetramorium $\mathrm{sp} .04$ & Hosang & Tetramorium sp.06 \\
\hline Tetramorium sp.01 & Bos & Tetramorium sp.10 \\
\hline Odontomachus sp.01 & Hosang & Odontomachus simillimus \\
\hline Pachycondyla sp.\#0? (Queen) & Hosang & Pachycondyla sp.01 \\
\hline
\end{tabular}


Chapter 3 - Long-term change of ant community structure

Table S2. Questionnaire used to record data on agricultural management and environmental conditions during the first and second samplings.

\begin{tabular}{|c|c|c|c|c|}
\hline No & Items & \multicolumn{2}{|c|}{ Data } & Note \\
\hline A. & Agricultural management & Frequency & Type & \\
\hline 1. & Pesticide application & & & \\
\hline & Insecticide & $\begin{array}{l}\text { 2001/2003: } \\
\text { 2009: }\end{array}$ & $\begin{array}{l}\text { 2001/2003: } \\
\text { 2009: }\end{array}$ & time/year \\
\hline & Fungicide & $\begin{array}{l}\text { 2001/2003: } \\
\text { 2009: }\end{array}$ & $\begin{array}{l}\text { 2001/2003: } \\
\text { 2009: }\end{array}$ & time/year \\
\hline 2. & Fertilizer & & & \\
\hline & Fertilizer & $\begin{array}{l}\text { 2001/2003: } \\
\text { 2009: }\end{array}$ & $\begin{array}{l}\text { 2001/2003: } \\
\text { 2009: }\end{array}$ & time/year \\
\hline 3. & Weed management & & & \\
\hline & Herbicide & $\begin{array}{l}\text { 2001/2003: } \\
\text { 2009: }\end{array}$ & $\begin{array}{l}\text { 2001/2003: } \\
\text { 2009: }\end{array}$ & time/year \\
\hline & Manual & $\begin{array}{l}\text { 2001/2003: } \\
\text { 2009: }\end{array}$ & $\begin{array}{l}\text { 2001/2003: } \\
\text { 2009: }\end{array}$ & time/year \\
\hline 4. & Pruning & & & \\
\hline & Manual & $\begin{array}{l}\text { 2001/2003: } \\
\text { 2009: }\end{array}$ & $\begin{array}{l}\text { 2001/2003: } \\
\text { 2009: }\end{array}$ & time/year \\
\hline B. & Habitat condition & $2001 / 2003$ & 2009 & \\
\hline 5. & Cacao field condition & & & $\begin{array}{l}\text { Not changed (0) } \\
\text { or Changed (1) }\end{array}$ \\
\hline
\end{tabular}


Chapter 3 - Long-term change of ant community structure

Table S3. Number of individuals per ant species comparing results from canopy fogging samples of Hosang in 2001 and Bos in 2003 and re-sampling by Rizali et al. in 2009. Morphospecies were based on the different of external morphology.

\begin{tabular}{|c|c|c|c|c|c|}
\hline \multirow{2}{*}{ No. } & \multirow{2}{*}{ Species } & \multicolumn{2}{|c|}{ Rainforest-poor Palolo region } & \multicolumn{2}{|c|}{ Rainforest-rich Kulawi region } \\
\hline & & 2001 & 2009 & 2003 & 2009 \\
\hline \multicolumn{6}{|c|}{ Dolichoderinae } \\
\hline 1. & Dolichoderus sp.01 & 17 & 415 & 466 & 2391 \\
\hline 2. & Dolichoderus sp.02 & & & 24 & \\
\hline 3. & Dolichoderus thoracicus & 15780 & 43244 & & \\
\hline 4. & Philidris cordata & 9129 & 21540 & & 32549 \\
\hline 5. & Tapinoma melanocephalum & 82 & 13 & 70 & 4 \\
\hline 6. & Tapinoma sp.01 & 1 & 24 & & \\
\hline 7. & Technomyrmex albipes & 937 & 1810 & & 1572 \\
\hline 8. & Technomyrmex sp.01 & 16 & & 3 & 11 \\
\hline 9. & Technomyrmex sp.02 & 2 & & & \\
\hline \multicolumn{6}{|c|}{ Formicinae } \\
\hline 10. & Anoplolepis gracilipes & 399 & 206 & 288 & 279 \\
\hline 11. & Calomyrmex sp.01 & 43 & & & \\
\hline 12. & Calomyrmex sp.02 & 1 & & & \\
\hline 13. & Camponotus recticulatus & 5 & 60 & 96 & 14 \\
\hline 14. & Camponotus sp.01 & 58 & 48 & 21 & 8 \\
\hline 15. & Camponotus sp.02 & 1 & & 5 & \\
\hline 16. & Echinopla lineata & & 1 & 10 & 13 \\
\hline 17. & Oecophylla smaragdina & 12527 & 363 & 26 & 576 \\
\hline 18. & Paratrechina sp.01 & 79 & 84 & 436 & 207 \\
\hline 19. & Paratrechina sp. 02 & 1 & & & \\
\hline 20. & Paratrechina sp.03 & 3 & & 140 & \\
\hline 21. & Paratrechina sp.04 & & & 10 & \\
\hline 22. & Paratrechina sp.05 & & & 4 & \\
\hline 23. & Polyrhachis abdominalis & & 1 & 8 & 12 \\
\hline 24. & Polyrhachis dives & 26 & 7 & 30 & 29 \\
\hline 25. & Polyrhachis sp.01 & 160 & 40 & 123 & 226 \\
\hline 26. & Polyrhachis sp.02 & 118 & 15 & 93 & 59 \\
\hline 27. & Polyrhachis sp.04 & & & 40 & 2 \\
\hline 28. & Polyrhachis sp.06 & 120 & 62 & 146 & 28 \\
\hline 29. & Polyrhachis sp.07 & 9 & 1 & & \\
\hline 30. & Polyrhachis sp.08 & 9 & 1 & 2 & \\
\hline 31. & Polyrhachis sp.09 & & & 1 & \\
\hline 32. & Polyrhachis sp.10 & 3 & & & \\
\hline 33. & Polyrhachis sp.11 & 1 & & & \\
\hline 34. & Polyrhachis sp. 13 & 1 & & & \\
\hline 35. & Polyrhachis sp. 14 & 3 & & 1 & \\
\hline 36. & Polyrhachis sp.15 & & & 2 & \\
\hline 37. & Polyrhachis sp.16 & 11 & & 104 & \\
\hline 38. & Polyrhachis sp.17 & & & 8 & \\
\hline 39. & Polyrhachis sp. 19 & 3 & & & \\
\hline 40. & Polyrhachis sp.20 & 6 & & & \\
\hline 41. & Pseudolasius sp.01 & & 5 & & \\
\hline 42. & Pseudolasius sp.02 & & 62 & & 36 \\
\hline
\end{tabular}


Chapter 3 - Long-term change of ant community structure

\begin{tabular}{|c|c|c|c|c|c|}
\hline \multirow{2}{*}{ No. } & \multirow{2}{*}{ Species } & \multicolumn{2}{|c|}{ Rainforest-poor Palolo region } & \multicolumn{2}{|c|}{ Rainforest-rich Kulawi region } \\
\hline & & 2001 & 2009 & 2003 & 2009 \\
\hline \multicolumn{6}{|c|}{ Myrmicinae } \\
\hline 43. & Cataulacus sp.01 & & & 4 & \\
\hline 44. & Crematogaster sp.01 & 362 & 1013 & 177 & 206 \\
\hline 45. & Crematogaster sp.02 & & & 26 & \\
\hline 46. & Crematogaster sp.03 & 15 & 253 & 96 & 538 \\
\hline 47. & Crematogaster sp.04 & 113 & & & \\
\hline 48. & Crematogaster sp.05 & 161 & & & \\
\hline 49. & Crematogaster sp.06 & 4 & & & \\
\hline 50. & Crematogaster sp.08 & 4 & & & \\
\hline 51. & Crematogaster sp.09 & 3 & & & \\
\hline 52. & Monomorium floricola & 94 & 54 & & 273 \\
\hline 53. & Monomorium sp.01 & 2 & 6 & & \\
\hline 54. & Monomorium sp.03 & 2 & & & \\
\hline 55. & Paratopula sp.01 & & & 9 & \\
\hline 56. & Paratopula sp.02 & & & 2 & \\
\hline 57. & Phedologeton sp.02 & 2 & & & \\
\hline 58. & Pheidole sp.01 & 1 & & & \\
\hline 59. & Pheidole sp.02 & & & 1 & 1 \\
\hline 60. & Pheidole sp.03 & & 1 & & 2 \\
\hline 61. & Pheidole sp.04 & & 6 & & 3 \\
\hline 62. & Rhoptromyrmex sp.03 & 1 & & & \\
\hline 63. & Rhoptromyrmex sp.04 & 18 & & & \\
\hline 64. & Solenopsis geminata & & & & 20 \\
\hline 65. & Strumigenys sp.01 & & & & 8 \\
\hline 66. & Strumigenys sp.03 & 11 & & & \\
\hline 67. & Tetramorium bicarinatum & & 1 & & 3 \\
\hline 68. & Tetramorium pasificum & 4 & 31 & & 68 \\
\hline 69. & Tetramorium smithi & 11 & 2 & & \\
\hline 70. & Tetramorium sp.01 & & & & 1 \\
\hline 71. & Tetramorium sp.02 & & & 10 & \\
\hline 72. & Tetramorium sp.03 & & & & 1 \\
\hline 73. & Tetramorium sp.05 & 2 & & & \\
\hline 74. & Tetramorium sp.06 & 26 & 7 & 5 & 720 \\
\hline 75. & Tetramorium sp.09 & 1 & & & \\
\hline 76. & Tetramorium sp.10 & & & 10 & \\
\hline \multicolumn{6}{|c|}{ Ponerinae } \\
\hline 77. & Gnamptogenys sp.01 & & & 24 & \\
\hline 78. & Hypoponera sp.01 & 1 & & & \\
\hline 79. & Hypoponera sp.02 & 1 & & & \\
\hline 80. & Odontomachus simillimus & 1 & 1 & & \\
\hline 81. & Pachycondyla sp.01 & 3 & & 1 & \\
\hline 82. & Pachycondyla sp.02 & 1 & & & \\
\hline 83. & Pachycondyla sp.04 & & & & 1 \\
\hline 84. & Platythyrea sp.01 & 1 & & & \\
\hline 85. & Ponera sp.01 & & & & 1 \\
\hline \multicolumn{6}{|c|}{ Pseudomyrmicinae } \\
\hline 86. & Tetraponera sp.01 & 9 & 52 & 18 & 13 \\
\hline 87. & Tetraponera sp.02 & & 1 & & \\
\hline
\end{tabular}


Chapter 3 - Long-term change of ant community structure

Table S4. Ant species collected from control and treatment plots using hand collecting (HC) and baiting (B) method.

\begin{tabular}{|c|c|c|c|c|c|c|c|}
\hline \multirow{2}{*}{ No } & \multirow{2}{*}{ Species } & \multicolumn{3}{|c|}{ Control } & \multicolumn{3}{|c|}{ Treatment } \\
\hline & & HC & $\mathbf{B}$ & Total & $\mathrm{HC}$ & $\mathbf{B}$ & Total \\
\hline \multicolumn{8}{|c|}{ Dolichoderinae } \\
\hline 1. & Dolichoderus sp.01 & + & & + & + & & + \\
\hline 2. & Tapinoma melanocephalum & + & + & + & + & + & + \\
\hline \multicolumn{8}{|c|}{ Formicinae } \\
\hline 3. & Anoplolepis gracilipes & + & + & + & + & + & + \\
\hline 4. & Camponotus recticulatus & + & + & + & + & + & + \\
\hline 5. & Camponotus sp.02 & & + & + & + & & + \\
\hline 6. & Oecophylla smaragdina & + & & + & & & \\
\hline 7. & Paratrechina sp.01 & + & + & + & + & & + \\
\hline 8. & Polyrhachis dives & + & & + & + & + & + \\
\hline 9. & Polyrhachis sp.04 & + & & + & & & \\
\hline \multicolumn{8}{|c|}{ Myrmicinae } \\
\hline 10. & Crematogaster sp.01 & + & + & + & + & + & + \\
\hline 11. & Monomorium floricola & + & + & + & + & + & + \\
\hline 12. & Monomorium sp.01 & + & & + & & & \\
\hline 13. & Pheidole sp.01 & + & + & + & + & + & + \\
\hline 14. & Pheidole sp.03 & + & & + & + & & + \\
\hline 15. & Tetramorium sp.01 & + & & + & & & \\
\hline 16. & Tetramorium sp.02 & + & + & + & & + & + \\
\hline \multicolumn{8}{|c|}{ Pseudomyrmicinae } \\
\hline 17. & Tetraponera sp.01 & + & & + & + & & + \\
\hline
\end{tabular}




\section{Appendix Figures}

Figure S1. Map of study sites located in the border of Lore Lindu National Park, Central Sulawesi, Indonesia. Plots in Palolo (solid triangle) and in Kulawi (solid circle).

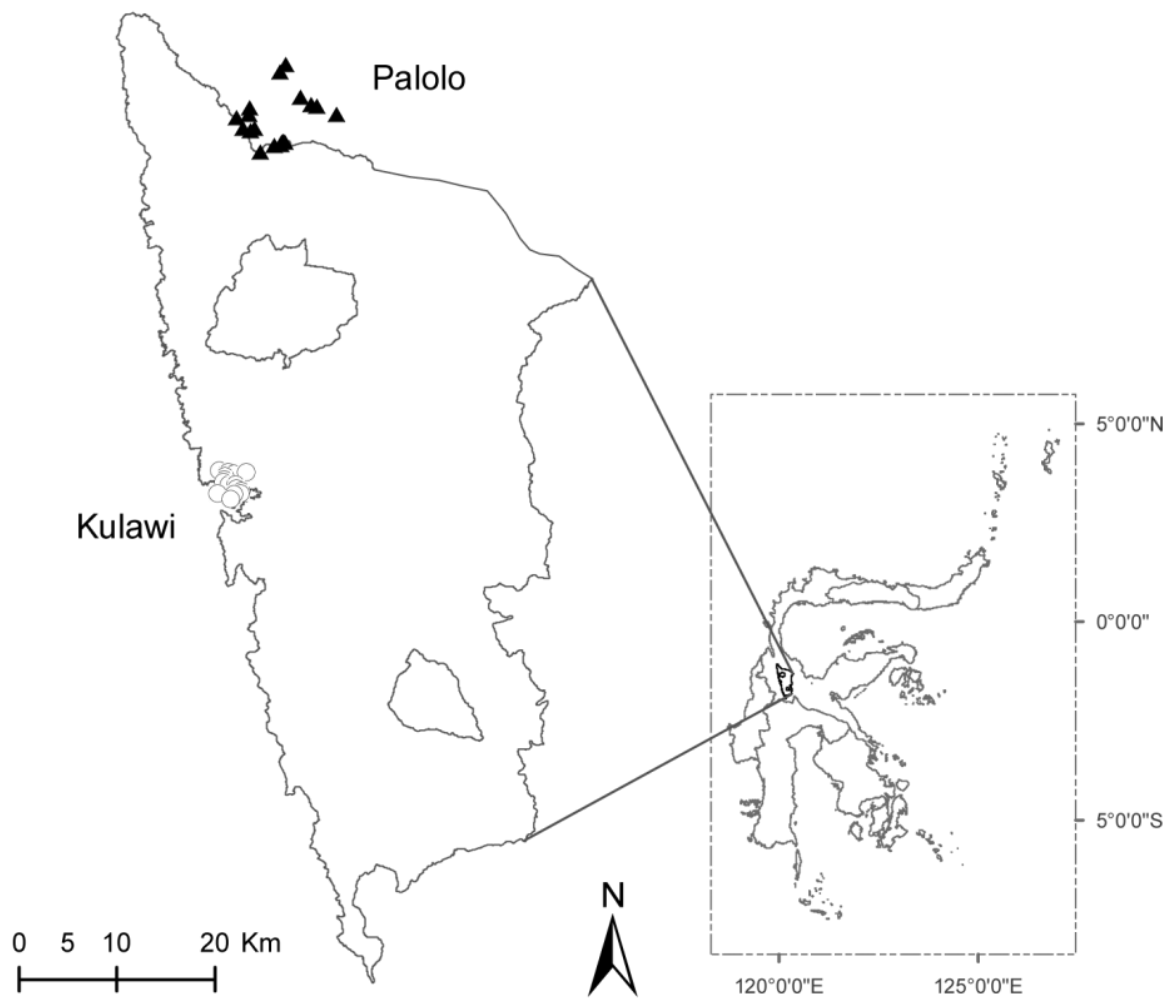


Figure S2. Plot design for pesticide experiment. Eight plots were groups into four control plots (open circle) and four treatment plots (closed circle). Each plot size about $8 \mathrm{~m} \times 8 \mathrm{~m}$ consists of nine cacao trees.

$8 \mathrm{~m}$\begin{tabular}{|lll|lll|lll|lll|}
\hline 0 & 0 & 0 & 0 & 0 & 0 & 0 & 0 & 0 & 0 & 0 & 0 \\
0 & 0 & 0 & 0 & 0 & 0 & 0 & 0 & 0 & 0 & 0 & 0 \\
0 & 0 & 0 & 0 & 0 & 0 & 0 & 0 & 0 & 0 & 0 & 0 \\
\hline 0 & 0 & 0 & 0 & 0 & 0 & 0 & 0 & 0 & 0 & 0 & 0 \\
0 & 0 & 0 & 0 & 0 & 0 & 0 & 0 & 0 & 0 & 0 & 0 \\
0 & 0 & 0 & 0 & 0 & 0 & 0 & 0 & 0 & 0 & 0 & 0 \\
\hline
\end{tabular}


Figure S3. Ant species composition in the rainforest-poor Palolo region between (a) 2001 and (b) 2009. Number of cacao trees occupied is based on total number of cacao trees from which that species was recorded (irrespective of the plot).

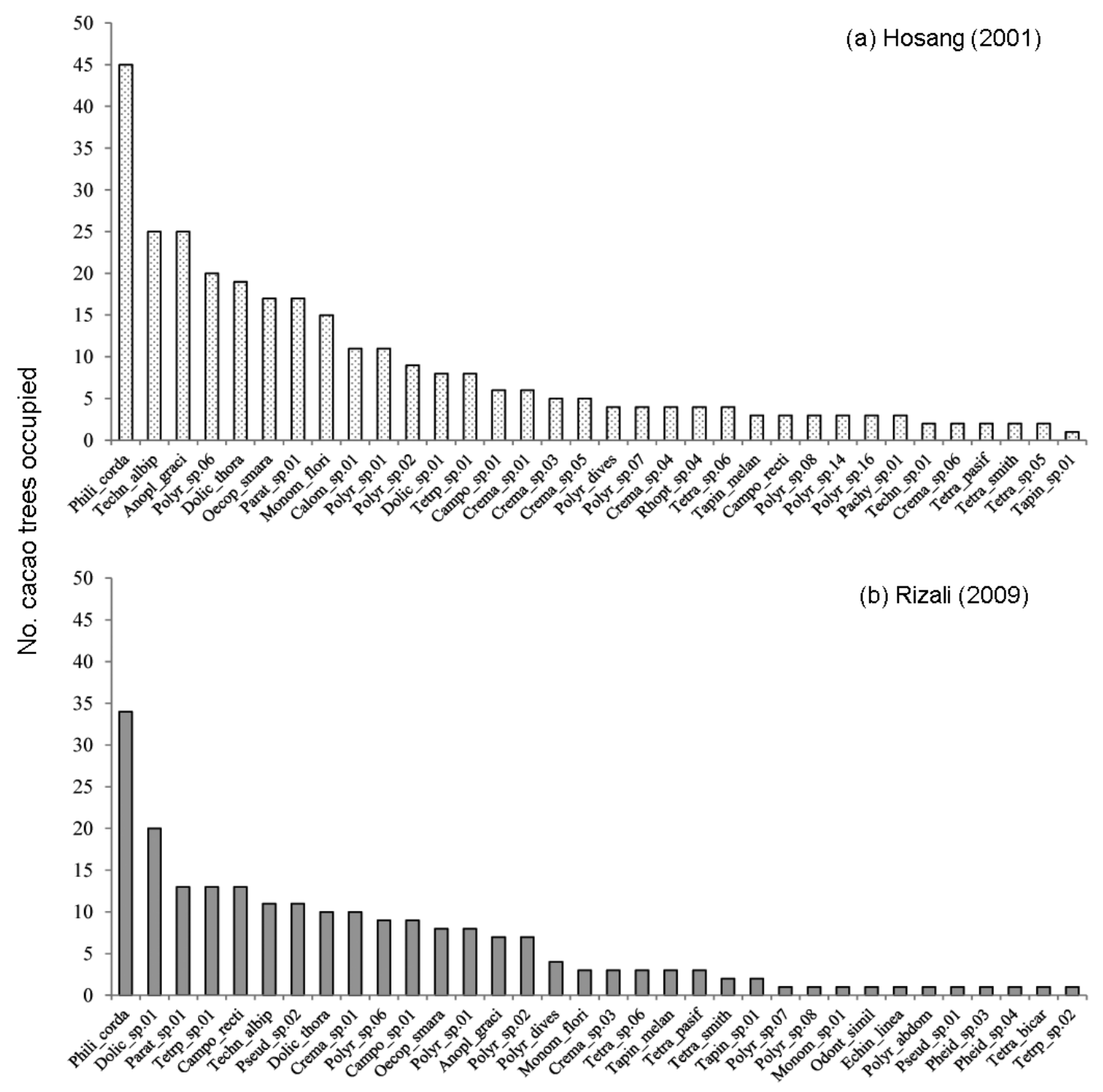


Figure S4. Ant species composition in the rainforest-rich Kulawi region between (a) 2003 and (b) 2009. Number of cacao trees occupied is based on total number of cacao trees from which that species was recorded (irrespective of the plot).

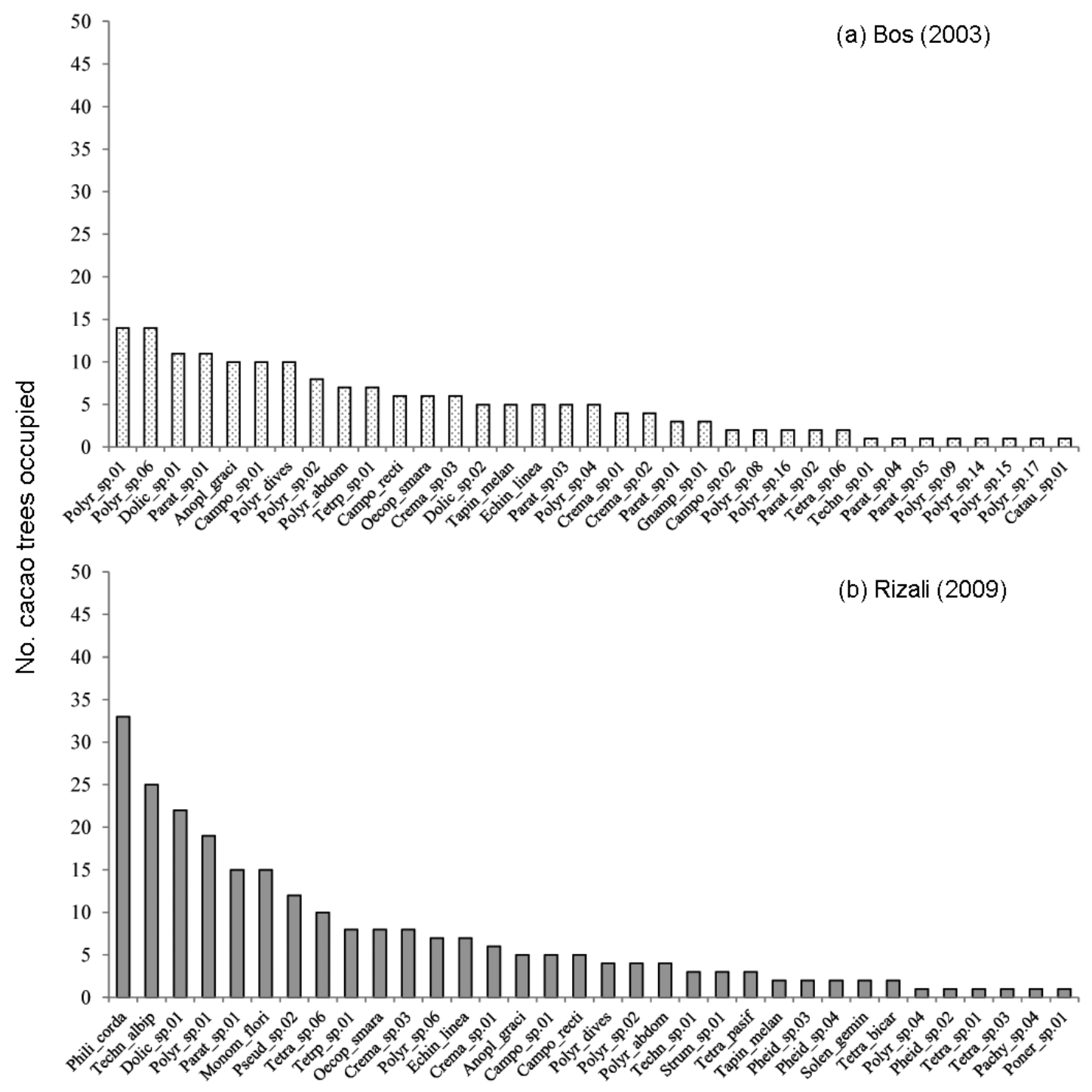




\section{Chapter 4}

Separating effect of species identity and species richness on ant predation

Akhmad Rizali, Yann Clough, Teja Tscharntke

In preparation for submission 


\section{ABSTRACT}

Ants are abundant in natural and managed tropical ecosystems and can impact herbivorous arthropods as well as plant pathogens. While it has been shown for plants that the diversity of communities can result in improved ecosystem functioning, how the species richness of ants affects ecosystem services and disservices such as pest control, disease transmission and invasion of potentially deleterious ant species is unknown. Here, we use experimentally enhanced natural gradients in ant species richness on 100 cacao trees in a plantation in Central Sulawesi, Indonesia, to analyze the effect of ant species identity and species richness on predation of herbivores, spread of phytopathogens, and resistance to an invasive ant species, Philidris cordata. Ant species richness had no significant effect in improving detection of prey in the predation experiment, and was not associated with a reduction in the incidence of pests. Incidence of the disease caused by $P$. palmivora was higher in ant species rich trees. Identity of the dominant ant species affected incidence of pests and diseases as well as predation pressure. Establishment of the ant $P$. cordata occurred irrespective of ant species richness or the identity of the dominant ant species. In conclusion, both ant species richness and identity affect ecosystem services and disservices delivered by the ant community, with results suggesting that ant identity can be more important than richness in determining biological control of pests.

\section{Keywords}

Anoplolepis gracilipes, ant predation, invasive species, Philidris cordata, species identity, species richness 


\section{INTRODUCTION}

Both the identity and the diversity of species present in a community have been shown to be important in affecting the function exerted by the community. Most evidence comes from pioneering research on the primary productivity of plant communities. Recently, studies have attempted at elucidating how predator diversity affects top-down control of lower trophic levels (e.g. Snyder et al., 2008). This research is important both for improving our understanding of food-webs and for contributing towards increased natural regulation of herbivory in managed systems. Results of a recent meta-analysis suggest that predator diversity is generally associated with lower herbivore numbers (Letourneau et al., 2009). However, existing studies generally involve exclusion of whole taxonomical or functional groups of predators, rather than investigating species richness within these groups. Since whole functional groups are rarely totally absent even in strongly disturbed systems, this limits the practical relevance of these results. Furthermore, this type of data does not enable to separate species identity and species richness effects, and thereby understand the drivers of diversity.

Ants are important components of most terrestrial ecosystems in terms of numbers and biomass. While in effect they span a gradient between cryptic herbivores and predators, it is expected that higher ant species richness contributes to increased stability and strength of trophic control of herbivores unattended by ants (Philpott \& Armbrecht, 2006). Much knowledge on ants and their interactions with other components of the ecosystem come from agroforestry systems such as coffee or cacao, where ants are expected to act as predators of arthropod pests. Other important functions for which ant species richness may be important is hampering the spread of plant disease, and biotic resistance to invasive ants, which are often ecologically and economically detrimental. 
There are several mechanisms by which ant diversity may be important. Ants differ substantially in resource use, with some species acquiring more energy from nectar or honeydew, or from different types of arthropods (Philpott et al., 2004). Foraging strategies and recruitment behaviors of ant species also differ (Hölldobler \& Wilson, 1990). Intraspecific differences in foraging or behavior may augment overall responses to herbivore communities as has been shown for spiders (Schmitz \& Suttle, 2001). Similar patterns have been suggested for ants (Philpott \& Armbrecht, 2006) but the evidence is still scarce (Gove, 2007). Biotic resistance to invasion is thought to act via reduced niche opportunity in species rich communities (Shea \& Chesson, 2002). Benefits of species diversity in functionality may thus be due to complementarity between species (Armbrecht et al., 2004).

Here, we attempt to separate the effect of ant species identity and species richness on (i) predation pressure and pest incidence, (ii) spread of Phytophthora palmivora in the cacao tree and total disease incidence, and (iii) the establishment success of a recently spreading, aggressive Dolichoderine ant species, Philidris cordata. We selected trees differing in ant species richness and identity of ants, including dominant species. In part of the trees, we manipulated the ant communities by setting up barriers to limit access of ground-nesting ants such as Anoplolepis gracilipes. The incidence of pests and diseases in the trees was monitored. We conducted a predation experiment using larvae of Corcyra cephalonica (Lepidoptera, Pyralidae) to compare the effect of ant species richness and treatment to predation success. The spread of $P$. palmivora from a standardized source inoculum, and establishment success of $P$. cordata nests were recorded. 


\section{METHODS}

\section{Study site}

Field experiments were conducted in cacao plantation in Ranteleda village, Palolo valley, Central Sulawesi, Indonesia (Fig. 1). The area of cacao plantation is about 6,000 $\mathrm{m}^{2}$ and located surrounding rice field with altitude 565 meter above sea level. Cacao trees were planted with regular distance $2.5 \mathrm{~m}$ x $2.5 \mathrm{~m}$, with shade trees Gliricidia sepium (dominant) and Durio zibethinus (rare). When the experiment was started, the age of cacao trees was 6 years. We selected a plot of $10 \times 10$ rows of cacao trees $(n=100)$, the average $\pm \mathrm{SD}$ of stem diameter is $8.8 \pm 1.56 \mathrm{~cm}$ and height is $383.5 \pm 44.89 \mathrm{~cm}$.

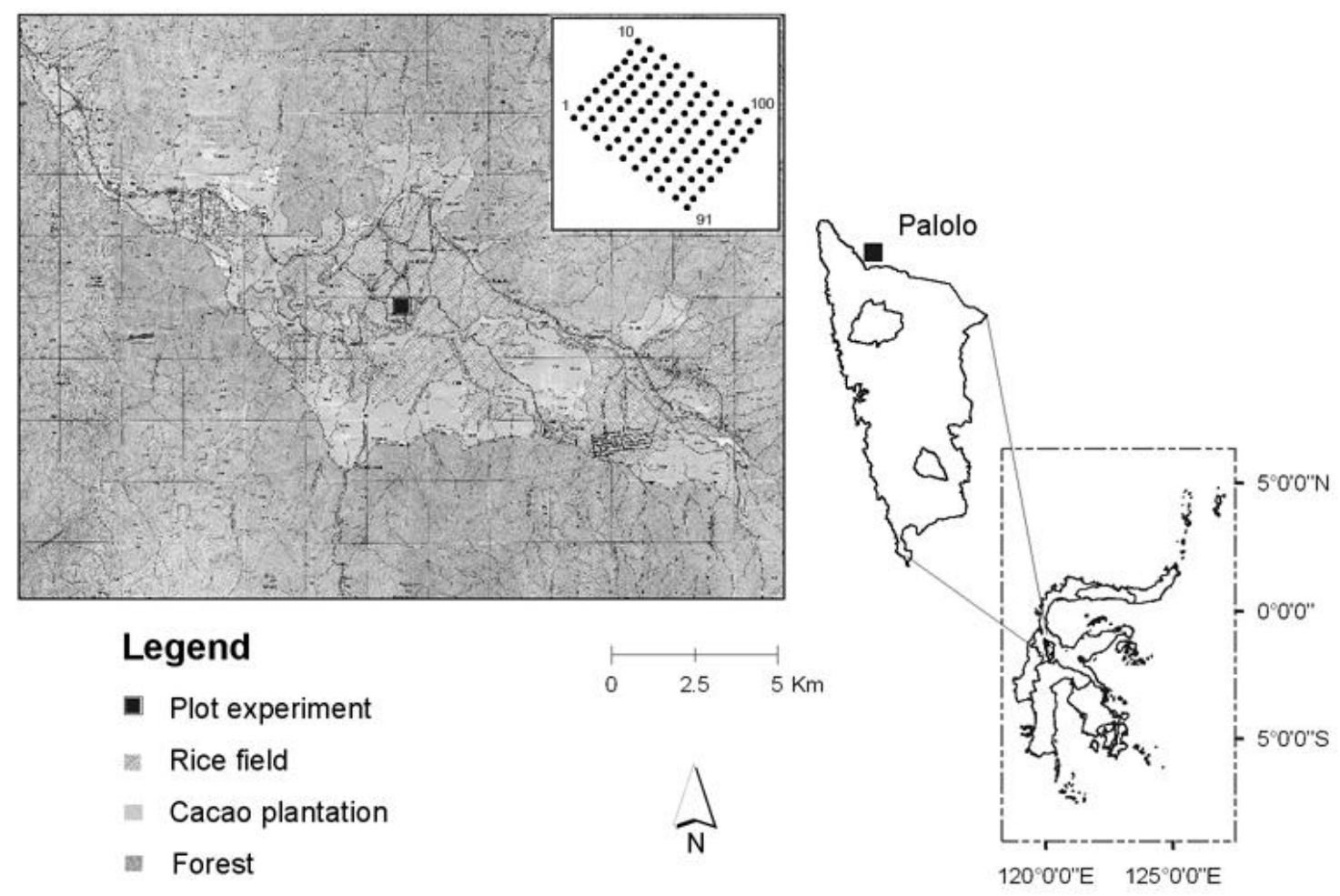

Figure 1. Map of the experimental site surrounded by rice fields in Ranteleda village, Palolo valley. A hundred cacao trees were regularly selected for predation experiment (top right of landscape map). Palolo valley is located several kilometers from the border of Lore Lindu National Park, Central Sulawesi, Indonesia (right side) 


\section{Ant diversity gradient}

We conducted ant surveys on all 100 cacao trees (Fig. 1, inset) to get information about ant diversity in different months i.e. in October 2009 (before pruning) and in February 2010 (after pruning). Ants were surveyed intensively by searching and collecting ants in different microhabitats, which is the best method to record all of ant species inhabiting an area (Bestelmeyer et al., 2000). Ants were identified to genus (Bolton, 1994) and further sorted to units according to their external morphology (morphospecies) (Lattke, 2000). Some ants were identified until species level based on reliable digital resources (e.g., http://www.antweb.org). The dominant ant species on the trees were A. gracilipes, Crematogaster sp.01 and Monomorium floricola, with A. gracilipes being the only dominant ground-nesting ant species.

Based on ant diversity collected from February 2010, we selected trees to obtain a gradient of ant species richness. The gradient was selected to hold for trees dominated by Crematogaster sp.01, Monomorium floricola, or both of these species independently, to allow the separation of effects due to identity of the dominant ant from those due to the species richness of the community (Table 1). In addition to this, we randomly selected 45 trees and applied a tanglefoot barrier (Tanglefoot ${ }^{\mathrm{TM}}$; Fig. 2) to prevent access by groundnesting ants such as A. gracilipes. The tanglefoot barrier was renewed every month, weeds which may connector the cacao trees to the ground were cut, and the cacao trees pruned to disconnect the canopies.

Ants were monitored intensively by hand collecting and visual observation in April 2010 (after barrier treatment), May 2010 (before establishment of P. cordata) and August 2010 (after establishment of $P$. cordata). 


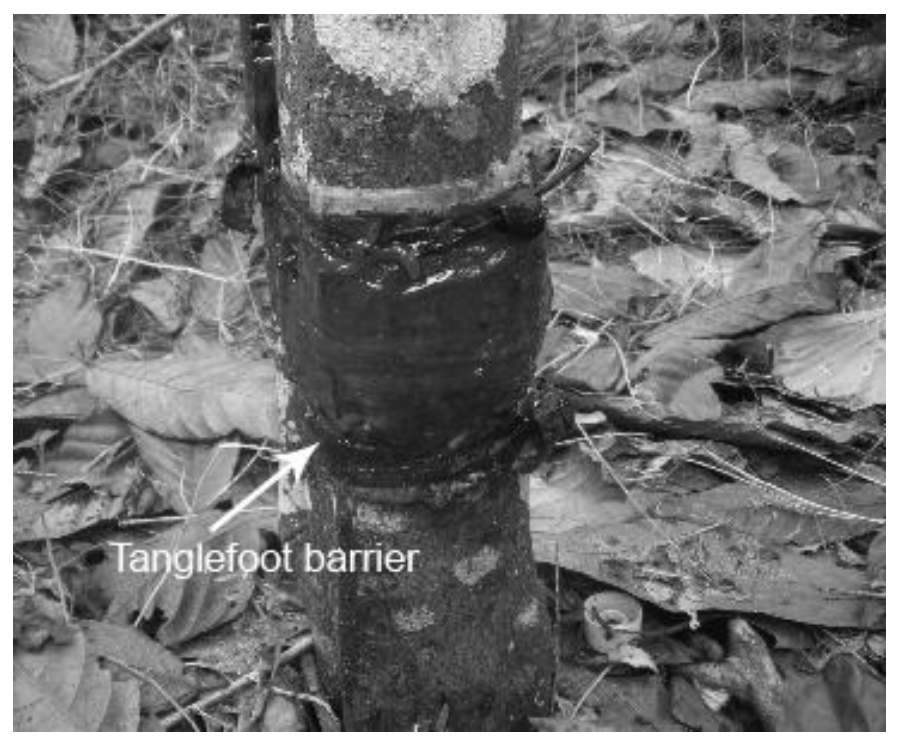

Figure 2. Treatment of tanglefoot barrier (using Tanglefoot ${ }^{\mathrm{TM}}$ ) to protect the cacao trees from ground-nesting ant such as A. gracilipes.

\section{Pest and disease incidence observation}

We observed pest and disease incidence on the 100 trees every two weeks, from April 2010 (after barrier treatment) until August 2010. We counted cacao fruits which suffered attack by Conopomorpha cramerella (Lepidoptera, Gracillariidae) or cacao pod borer (CPB) and were infected by Phytophthora palmivora or black pod disease (BPD).

\section{Black pod disease experiment}

Before treatment, we removed all of cacao fruits that infected by BPD. Then, two cacao pods bearing symptoms of infection by BPD were put in the intersection of branch with minimum distance about $50 \mathrm{~cm}$ from healthy fruit. After three weeks, we recorded the incidence of BPD on the pods on each cacao tree. 


\section{Predation experiment}

We conducted a predation experiment to study the effect of ant species diversity and species dominant on predation success in cacao plantation. In this experiment, we used larvae of the rice moth, Corcyra cephalonica (Lepidoptera: Pyralidae) from instar 3-4 as prey in the experiment (Fig. 3a-3b). On each cacao tree, two larvae were put in pod and in the branch. The predation success of each ant species and the time needed for discovering and attacking each larva were measured in this experiment. Observation was conducted during day and night, to cover the effect of both diurnal and nocturnal ant activity.
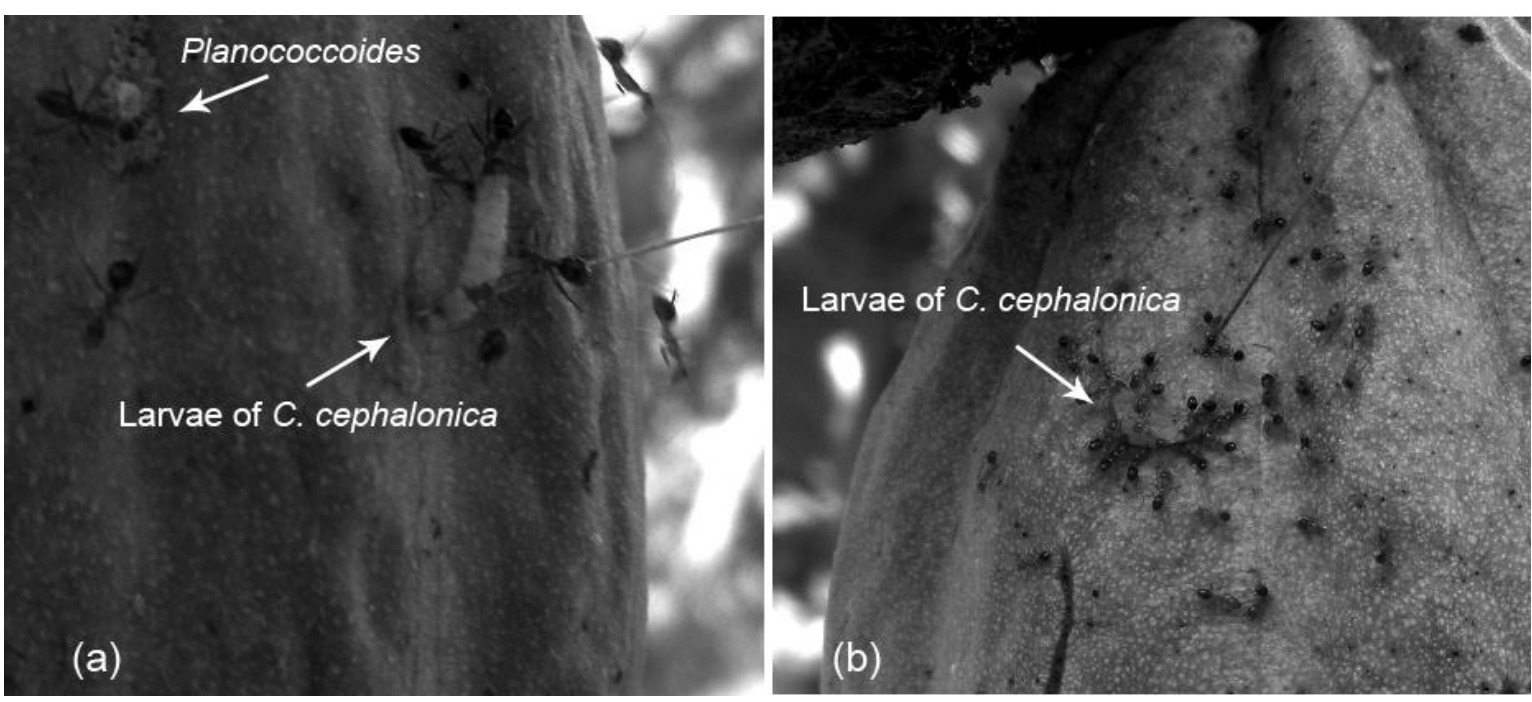

Figure 3. Predation experiment using larvae of C. cephalonica as prey. (a) A. gracilipes always found in cacao pod that invested by Planococcoides. (a) A. gracilipes and (b) P. cordata are attacking a larvae in cacao pod.

\section{Establishment success of $\boldsymbol{P}$. cordata}

In order to investigate the effect of ant communities on the establishment of new and potentially invasive ants, we imported from another cacao plantation nests of $P$. cordata and placed two nests per tree (Fig. 4) on 22 randomly selected cacao trees without tanglefoot barrier and 23 cacao trees with tanglefoot barrier. The nests were put in the 
branch, together with cacao fruit infested by the preferred homopteran trophobionts (Delabie, 2001) of this ant species (Planococcoides).

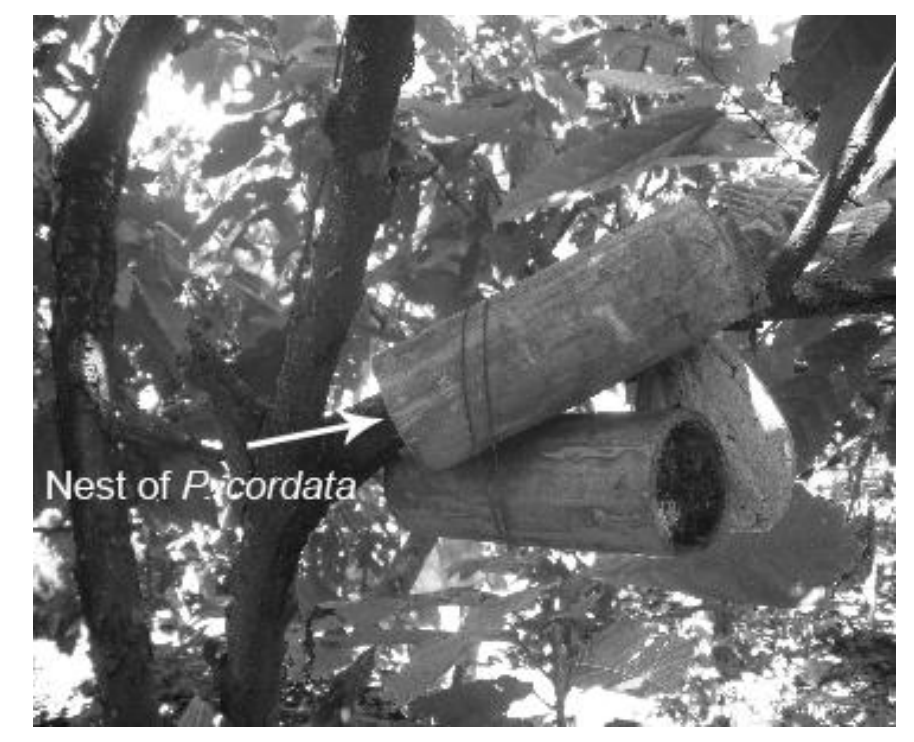

Figure 4. Introducing P. cordata by hanging two nests in the branch of cacao tree.

\section{Statistical analyses}

To analyze the effect of ant species identity and species richness on pest and diseases incidence, we used generalized linear mixed models (GLMM). We used models for proportion data. In addition to the fixed effects (see below), we included tree identity as a random grouping factor in order to associate the repeated measurements of ant diversity and pest and disease incidence to the corresponding tree and avoid pseudoreplication. Data recorded after introduction of $P$. cordata in trees selected for invasive ant establishment were not used in these analyses.

We fitted several models per response variable. The first model had only ant species richness as an explanatory variable. The second model had ant species richness, as well as the presence/absence of the dominant ants M. floricola, Crematogaster sp.01 and 
A. gracilipes. This second model was simplified by computing all possible subset models and selecting the model with the lowest Akaike information criteria (AIC).

We used generalized linear models (GLM) to analyze the effect of ant species identity and species richness on predation pressure. The response variable was proportion of attacked larvae. As above, we fitted a first model containing only ant species richness as an explanatory variable, followed by a second model including ant species richness and the presence/absence of the dominant ants M. floricola, Crematogaster sp.01 and A. gracilipes.

GLMM was also used to analyze the effect of ant species identity and species richness on spread of $P$. palmivora. In this case, we used data after establishment of $P$. cordata due to experiment was conducted after establishment. All analyses were conducted using R software (R Development Core Team, 2011) and the package lme4.

\section{RESULTS}

\section{Ant diversity from a hundred cacao trees}

Across all five ant survey rounds for the a hundred cacao trees, we recorded 27 ant species (Table 1). Species A. gracilipes, M. floricola and Crematogaster sp.01 were the most dominant species in this cacao plantation (Table 1, Fig. S1-S5). We found different range of ant diversity from 100 cacao trees between preliminary observation (S1 and S2) and after treatment of tanglefoot barrier (S3 and S4) and after establishment of P. cordata (S5) (Fig. 5). However, the range of ant diversity after treatment of tanglefoot and establishment of $P$. cordata were similar from one to eight ant species (Fig. 5). 
Table 1. Ant diversity in 100 cacao trees from preliminary observation (S1: before pruning and S2: after pruning), after tanglefoot barrier (S3), before establishment of P. cordata (S4) and after establishment of $P$. cordata (S5). Data is presented based on total of cacao trees that ant species collected from 100 cacao trees.

\begin{tabular}{|c|c|c|c|c|c|c|}
\hline No & Species & S1 & S2 & S3 & S4 & S5 \\
\hline & Dolichoderinae & & & & & \\
\hline 1. & Dolichoderus sp.01 & 44 & 43 & 24 & 21 & 19 \\
\hline 2. & Dolichoderus sp.02 & 2 & 4 & & & \\
\hline 3. & Philidris cordata & & & & & 53 \\
\hline 4. & Tapinoma melanocephalum & 13 & 10 & 6 & 11 & 12 \\
\hline 5. & Tapinoma sp.01 & 4 & 1 & & 1 & \\
\hline \multirow[t]{2}{*}{6.} & Technomyrmex sp.01 & 21 & 22 & 16 & 15 & 5 \\
\hline & Formicinae & & & & & \\
\hline 7. & Anoplolepis gracilipes & 99 & 100 & 65 & 69 & 43 \\
\hline 8. & Camponotus recticulatus & 36 & 69 & 26 & 45 & 48 \\
\hline 9. & Camponotus sp.01 & 3 & 2 & 2 & 1 & 1 \\
\hline 10. & Echinopla lineata & 16 & 11 & 5 & 3 & 4 \\
\hline 11. & Oecophylla smaragdina & 1 & 1 & & & \\
\hline 12. & Paratrechina sp.01 & 18 & 10 & 6 & 8 & 17 \\
\hline 13. & Polyrachis abdominalis & & & 1 & 1 & \\
\hline 14. & Polyrachis dives & 68 & 49 & 41 & 41 & 29 \\
\hline 15. & Polyrachis sp.02 & 8 & 3 & 5 & 4 & 3 \\
\hline 16. & Polyrachis sp.03 & 2 & & 2 & & \\
\hline \multirow[t]{2}{*}{17.} & Polyrachis sp.04 & 1 & 5 & 5 & 7 & 13 \\
\hline & Myrmicinae & & & & & \\
\hline 18. & Crematogaster sp.01 & 39 & 43 & 47 & 46 & 30 \\
\hline 19. & Crematogaster sp.02 & & & & & 1 \\
\hline 20. & Monomorium floricola & 62 & 68 & 65 & 70 & 32 \\
\hline 21. & Monomorium sp.01 & 1 & & & & \\
\hline 22. & Pheidole sp.01 & 2 & & 1 & 3 & 1 \\
\hline 23. & Pheidole sp.03 & & & & 2 & 4 \\
\hline 24. & Solenopsis geminata & & & & 1 & \\
\hline 25. & Tetramorium sp.01 & 12 & 14 & 13 & 14 & 8 \\
\hline \multirow[t]{2}{*}{26.} & Tetramorium sp.02 & 3 & 1 & 7 & 9 & 6 \\
\hline & Pseudomyrmicinae & & & & & \\
\hline 27. & Tetraponera sp.01 & 20 & 25 & 15 & 18 & 12 \\
\hline
\end{tabular}




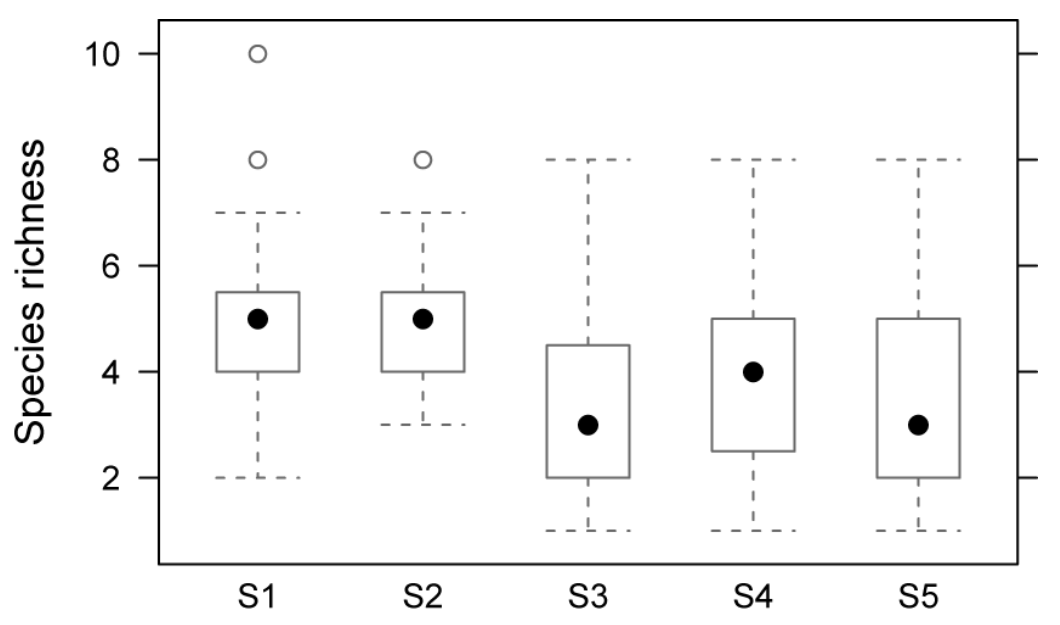

Figure 5. Box plot the range of ant species richness in 100 cacao trees from preliminary observation (S1: before pruning and S2: after pruning), after tanglefoot barrier (S3), before establishment of $P$. cordata (S4) and after establishment of $P$. cordata (S5).

\section{Pest and disease incidence}

Ant species richness had contrasting effects on incidence of pest and disease in cacao plantation (Fig. 6). Our analysis showed that ant species richness did not influence incidence of pest $(\mathrm{CPB})$ based on both of first model $(\mathrm{z}=0.247, \mathrm{P}=0.805, \mathrm{AIC}=473.5)$ and second model $(\mathrm{z}=-0.555, \mathrm{P}=0.579, \mathrm{AIC}=467.2)$. Nevertheless, we found that the presence of dominant ant, $M$. floricola significantly influence $(\mathrm{z}=2.820, \mathrm{P}=0.005)$ incidence of $\mathrm{CPB}$ in cacao plantation.

In contrast, species richness significantly affect incidence of disease (BPD) based on both the first model $(\mathrm{z}=3.477, \mathrm{P}<0.001, \mathrm{AIC}=1266)$ and the second model $(\mathrm{z}=2.582$, $\mathrm{P}=0.009, \mathrm{AIC}=1262)$. Furthermore, ant species identity, M. floricola $(\mathrm{z}=2.044, \mathrm{P}=0.041)$ also affected incidence of $P$. palmivora or BPD. 


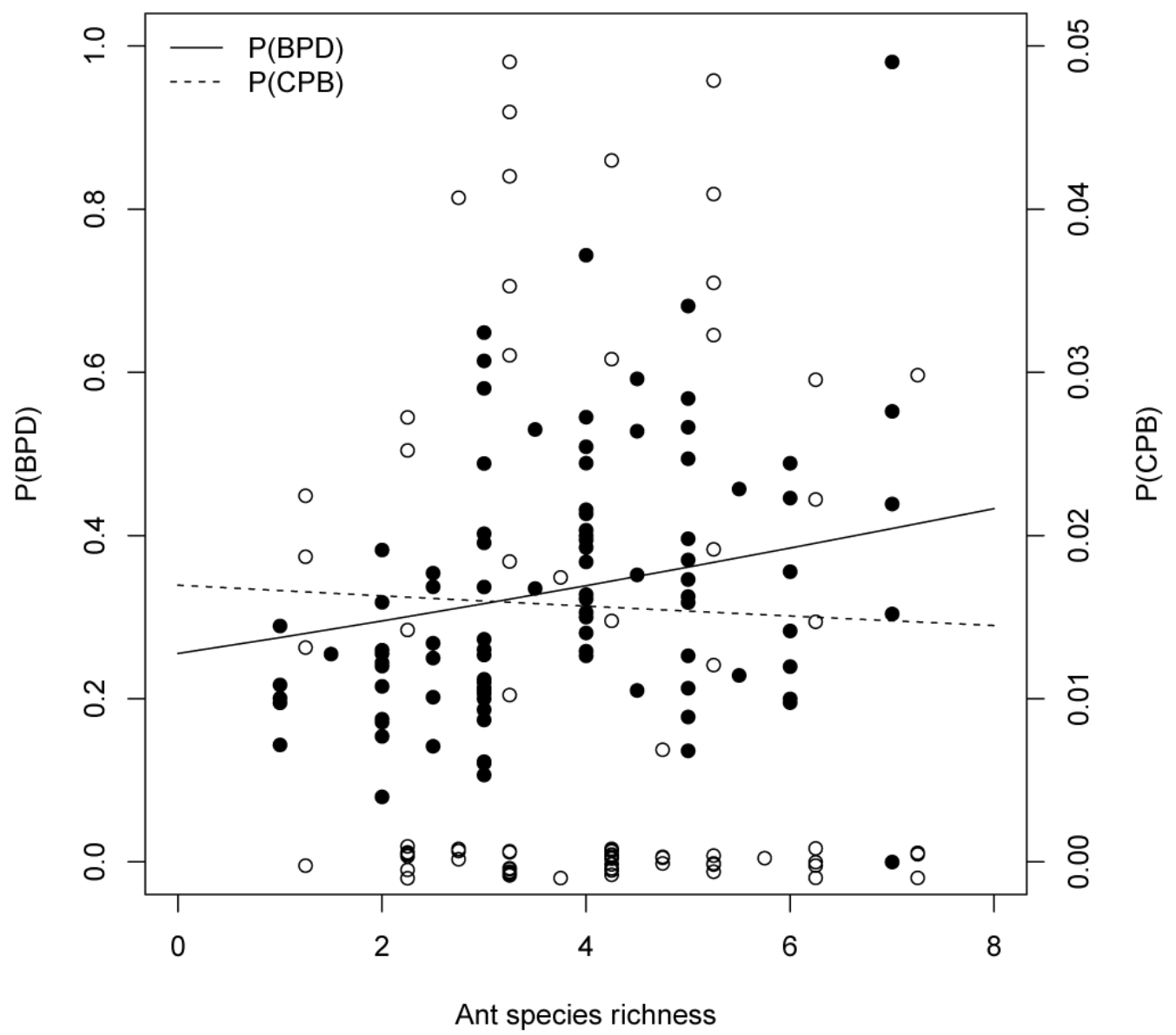

Figure 6. Relationship between ant species richness and incidence of cacao pod borer (CPB) and black pod diseases (BPD) in cacao plantation based on field observation.

\section{Predation experiment}

Our experiment showed that ant species richness had no effect on predation pressure based on both the first $(\mathrm{z}=0.462, \mathrm{P}=0.644$, $\mathrm{AIC}=133.29)$ and the second model $(\mathrm{z}=-1.256, \mathrm{P}=0.209, \mathrm{AIC}=126.98)$. We found 13 ant species to attack prey during the experiment both in day and night observation (Table 2). The presence of A. gracilipes $(\mathrm{z}=3.378, \mathrm{P}<0.001)$ significantly increased the predation pressure. Another dominant ant i.e. Crematogaster sp.01 ( $\mathrm{z}=0.323, \mathrm{P}=0.746)$ and M. floricola $(\mathrm{z}=-0.197, \mathrm{P}=0.843)$ had no significant effects on predation pressure. 
Table 2. Ant species found attack prey during predation experiment. Data is presented based on total of cacao trees that ant species found attacking prey from 100 cacao trees

\begin{tabular}{|r|l|c|c|c|}
\hline No & Species & Day & Night & Total \\
\hline 1. & Anoplolepis gracilipes & 29 & 27 & 33 \\
\hline 2. & Camponotus recticulatus & 2 & & 2 \\
\hline 3. & Camponotus $\mathrm{sp} .01$ & & 1 & 1 \\
\hline 4. & Crematogaster sp.01 & 12 & 11 & 14 \\
\hline 5. & Dolichoderus sp.01 & 4 & & 4 \\
\hline 6. & Monomorium floricola & 9 & & 9 \\
\hline 7. & Paratrechina sp.01 & & 1 & 1 \\
\hline 8. & Pheidole sp.03 & 1 & 1 & 1 \\
\hline 9. & Philidris cordata & 45 & 53 & 53 \\
\hline 10. & Polyrachis dives & 1 & & 1 \\
\hline 11. & Polyrachis sp.04 & & 1 & 1 \\
\hline 12. & Tapinoma melanocephalum & 1 & & 1 \\
\hline 13. & Technomyrmex sp.01 & & 1 & 1 \\
\hline 14. & Tetramorium sp.01 & 1 & & 1 \\
\hline 15. & Tetramorium sp.02 & 1 & & 1 \\
\hline 16. & Tetraponera sp.01 & 1 & & 1 \\
\hline
\end{tabular}

\section{P. palmivora dissemination}

Our BPD experiment showed that ant species richness had no effect on spread of $P$. palmivora in cacao plantation based on first model $(\mathrm{z}=0.483, \mathrm{P}=0.629, \mathrm{AIC}=326.4)$ and second model $(\mathrm{z}=0.596, \mathrm{P}=0.551, \mathrm{AIC}=331.8)$. The same pattern also showed in ant species identity i.e. A. gracilipes $(\mathrm{z}=-0.502, \mathrm{P}=0.616), P$. cordata $(\mathrm{z}=1.136, \mathrm{P}=0.256)$, Crematogaster sp.01 ( $\mathrm{z}=0.442, \mathrm{P}=0.659)$ and M. floricola $(\mathrm{z}=0.328, \mathrm{P}=0.743)$ which had no effect on incidence of BPD.

\section{Establishment success of $\boldsymbol{P}$. cordata}

From 45 cacao trees in which nests of $P$. cordata were placed, only five cacao trees were not successfully colonized, making the analysis of contrasts between ant communities in terms of resistance to invasion impossible (Table 1). 


\section{DISCUSSION}

Our research revealed that ant species identity and species richness play different roles in influencing the incidence of pests and diseases in cacao plantation. Ant species identity seems more important than ant species richness in determining pest incidence. Both our observational data and predation experiment showed that ant species identity is strongly related to predation pressure, as suggested by Straub and Snyder (2006). However, although this pattern was not shown in our BPD experiment, ant species richness appears to affect positively the incidence of disease in cacao plantation. We expected that a higher species richness may (i) result in spatial compartimentation of the cacao trees and thereby lower the likelihood of disease spread (ii) result in decreased activity of other potential vectors. This seems not to be the case. The observed pattern may be due to the increased likelihood of having an ant species which is likely to vector the spores of $P$. palmivora (Evans, 1973) in communities that are species rich than in communities that are species poor, via a selection effect. The establishment of $P$. cordata, an aggressive and invasive ant species, and a potential vector of P. palmivora (Wielgoss et al., 2010), had no effect on spread of the diseases in study area maybe due to short period of establishment.

The dominant ant species $P$. cordata, A. gracilipes, Crematogaster sp.01, and $M$. floricola are potential predator against pests in cacao plantation. Our observation showed that M. floricola significantly influences incidence of CPB. And based on predation experiment, A. gracilipes significantly affects predation pressure. In this case, ant species identity is more important than ant species richness (Straub \& Snyder, 2006). Predation pressure appears to be more directly linked to ant activity (Gove, 2007), therefore increasing ant activity such as A. gracilipes seems impact on increasing predation pressure in cacao plantation (Way \& Khoo, 1992). However, species richness of predator had no effect on predation pressure maybe due to only single prey species, C. cephalonica that we 
were used in predation experiment. Theory predicts that the importance of a diverse predator community for predation pressure will increase in a diverse prey community (Wilby \& Thomas, 2002; Ives et al., 2005). Another experiment study also revealed that top-down pressure of pest generally strengthens with higher predator diversity with multiple prey species (Cardinale et al., 2003; Snyder et al., 2006). This may be a reason for the absence of a predator species richness effect in our experiment.

The effect of ant species richness and species identity on incidence of disease in cacao plantation differed between observation and experiment. Our experiment did not show the influence both of ant species richness and species identity on spread of $P$. palmivora. This might relate to decreasing the role of certain species on reducing the disease transmission such as A. gracilipes which has indirect effect on reducing incidence of BPD through excluding another ants which act as as vector of $P$. palmivora (McGregor \& Moxon, 1985). In addition, introducing $P$. cordata that may also act as vector of $P$. palmivora (Wielgoss et al., 2010) can compete with another dominant ant such as $A$. gracilipes, M. floricola and Crematogaster sp.01. P. cordata, a tent-building ant species, contributes to vertical spread of the pathogen by transporting contaminated soil within the cacao tree (Evans, 1973). Therefore, we expected that the occurrence of $P$. cordata might promote the occurrence of BPD in cacao plantation in long time establishment.

The introduction of aggressive and invasive ant species, $P$. cordata in cacao plantation was successful, regardless of the number of ant species already present on the tree. This species can well adapt in new habitat although dominated by another invasive species A. gracilipes. Based on our observation, this species can occupy another cacao trees which were not placed the nests. $P$. cordata only requires presence of homopteran trophobionts for establishment through trophobiosis (Delabie, 2001). As invasive species, this species has ability to occupy homogenized habitat and displace other ants (Holway $e t$ 
al., 2002; Wagner \& van Driesche, 2010), including the invasive species A. gracilipes and also its trophobiont (e.g. Planococcoides) which always found abundant with presence of A. gracilipes (Fig. 3) through apparent competition (Carvalheiro et al., 2008). Therefore, P. cordata has not to be considered for biological control agents due to the high risk for biodiversity and also crop yield (see Snyder \& Evans, 2006; van Lenteren et al., 2006).

\section{CONCLUSION}

Ant species identity affects the incidence of pests in cacao plantations more than ant species richness. Ant species richness had no significant effect in improving detection of prey in the predation experiment, and was not associated with a reduction in the incidence of pest damage. In contrast, ant species richness seems to affect the incidence of the disease caused by $P$. palmivora which was found higher in ant species rich trees. Identity of the dominant ant species affected incidence of pests and diseases as well as predation pressure. Both ant species richness and identity are associated with ecosystem services and disservices delivered by the ant community, but our results caution against using ant species richness as an indicator for ecosystem services.

\section{ACKNOWLEDGEMENTS}

Thanks to our field assistants (Fifin, Moh. Fadlan, Akhmad Syakur, and Hadiprayitno), laboratory assistants (Nur Ivon and Dewi Utaminingsih), and local assistants (Alfianus and Mardianus). Thanks also to Baharuddin, the owner of cacao plantation for experiment. Thanks to M. Khaidir, Indrawan, M. Rizki, Laode, and Rizal who help during predation experiments. Thanks to the Center for Tropical Forest Margin (CTFM), University of Tadulako who support the administration and transportation during field survey. This research project was funded by the German Science Foundation (DFG), 
University of Goettingen, Bogor Agricultural University (IPB), and Directorate General of Higher Education, Ministry of National Education of the Republic of Indonesia.

\section{REFERENCES}

Armbrecht, I., Perfecto, I. \& Vandermeer, J. (2004) Enigmatic biodiversity correlations: ant diversity responds to diverse resources. Science, 304, 284-286.

Bestelmeyer, B.T., Agosti, D., Alonso, L.E., Brandão, C.R.F., Brown, W.L., Jr., Delabie, J.H.C. \& Silvestre, R. (2000) Field techniques for the study of ground-dwelling ants: an overview, description, and evaluation. Ants: Standard Methods for Measuring and Monitoring Biodiversity (ed. by D. Agosti, J.D. Majer, L.E. Alonso and T.R. Schultz), pp. 122-144. Smithsonian Institution Press, Washington.

Bolton, B. (1994) Identification Guide to the Ant Genera of the World. Harvard University Press, Cambridge.

Cardinale, B.J., Harvey, C.T., Gross, K. \& Ives, A.R. (2003) Biodiversity and biocontrol: emergent impacts of a multi-enemy assemblage on pest suppression and crop yield in an agroecosystem. Ecology Letters, 6, 857-865.

Carvalheiro, L.G., Buckley, Y.M., Ventim, R., Fowler, S.V. \& Memmott, J. (2008) Apparent competition can compromise the safety of highly specific biocontrol agents. Ecology Letters, 11, 690-700.

Delabie, J.H.C. (2001) Trophobiosis between Formicidae and Hemiptera (Sternorrhyncha and Auchenorrhyncha): an overview. Neotropical Entomology, 30, 501-516.

Evans, H.C. (1973) Invertebrate vectors of Phytophthora palmivora, causing black pod disease of cocoa in Ghana. Annals of Applied Biology, 75, 331-345.

Gove, A.D. (2007) Ant biodiversity and the predatory function (A response to Philpott and Armbrecht, 2006). Ecological Entomology, 32, 435-436. 
Hölldobler, B. \& Wilson, E.O. (1990) The Ants. Harvard University Press, Cambridge.

Holway, D.A., Lach, L., Suarez, A.V., Tsutsui, N.D. \& Case, T.J. (2002) The causes and consequences of ant invasions. Annual Review of Ecology and Systematics, 33, $181-233$

Ives, A.R., Cardinale, B.J. \& Snyder, W.E. (2005) A synthesis of subdisciplines: predatorprey interactions, and biodiversity and ecosystem functioning. Ecology Letters, 8, 102-116.

Lattke, J.E. (2000) Specimen processing: building and curating an ant collection. Ants: Standard Methods for Measuring and Monitoring Biodiversity (ed. by D. Agosti, J.D. Majer, L.E. Alonso and T.R. Schultz), pp. 155-171. Smithsonian Institution Press, Washington.

Letourneau, D.K., Jedlicka, J.A., Bothwell, S.G. \& Moreno, C.R. (2009) Effects of natural enemy biodiversity on the suppression of arthropod herbivores in terrestrial ecosystems. Annual Review of Ecology, Evolution and Systematics, 40, 573-592.

McGregor, A.J. \& Moxon, J.E. (1985) Potential for biological control of tent building species of ants associated with Phytophthora palmivora pod rot of cacao in Papua New Guinea. Annals of Applied Biology, 107, 271-277.

Philpott, S.M. \& Armbrecht, I. (2006) Biodiversity in tropical agroforests and the ecological role of ants and ant diversity in predatory function. Ecological Entomology, 31, 369-377.

Philpott, S.M., Greenberg, R., Bichier, P. \& Perfecto, I. (2004) Impacts of major predators on tropical agroforest arthropods: comparisons within and across taxa. Oecologia, 140, 140-149.

R Development Core Team (2011) R: A language and environment for statistical computing. R Foundation for Statistical Computing. 
Schmitz, O.J. \& Suttle, K.B. (2001) Effects of top predator species on direct and indirect interactions in a food web. Ecology, 82, 2072-2081.

Shea, K. \& Chesson, P. (2002) Community ecology theory as a framework for biological invasions. Trends in Ecology \& Evolution, 17, 170-176.

Snyder, G.B., Finke, D.L. \& Snyder, W.E. (2008) Predator biodiversity strengthens aphid suppression across single- and multiple-species prey communities. Biological Control, 44, 52-60.

Snyder, W.E. \& Evans, E.W. (2006) Ecological effects of invasive arthropod generalist predators. Annual Review of Ecology, Evolution and Systematics, 37, 95-122.

Snyder, W.E., Snyder, G.B., Finke, D.L. \& Straub, C.S. (2006) Predator biodiversity strengthens herbivore suppression. Ecology Letters, 9, 789-796.

Straub, C.S. \& Snyder, W.E. (2006) Species identity dominates the relationship between predator biodiversity and herbivore suppression. Ecology, 87, 277-282.

van Lenteren, J.C., Bale, J., Bigler, F., Hokkanen, H.M.T. \& Loomans, A.J.M. (2006) Assessing risks of releasing exotic biological control agents of arthropod pests. Annual Review of Entomology, 51, 609-634.

Wagner, D.L. \& van Driesche, R.G. (2010) Threats posed to rare or endangered insects by invasions of nonnative species. Annual Review of Entomology, 55, 547-568.

Way, M.J. \& Khoo, K.C. (1992) Role of ants in pest-management. Annual Review of Entomology, 37, 479-503.

Wielgoss, A., Tscharntke, T., Buchori, D., Fiala, B. \& Clough, Y. (2010) Temperature and a dominant dolichoderine ant species affect ant diversity in Indonesian cacao plantations. Agriculture, Ecosystems \& Environment, 135, 253-259.

Wilby, A. \& Thomas, M.B. (2002) Natural enemy diversity and pest control: patterns of pest emergence with agricultural intensification. Ecology Letters, 5, 353-360. 


\section{SUPPORTING INFORMATION}

\section{Appendix Figure}

\section{Figure Legends}

Figure S1. Spatial occurrence of ant species in a hundred cacao trees from preliminary observation in October 2009 (before pruning)

Figure S2. Spatial occurrence of ant species in a hundred cacao trees from preliminary observation in February 2010 (after pruning)

Figure S3. Spatial occurrence of ant species in a hundred cacao trees after treatment of tanglefoot barrier in April 2010

Figure S4. Spatial occurrence of ant species in a hundred cacao trees before establishment of $P$. cordata in May 2010

Figure S5. Spatial occurrence of ant species in a hundred cacao trees after establishment of $P$. cordata in August 2010 

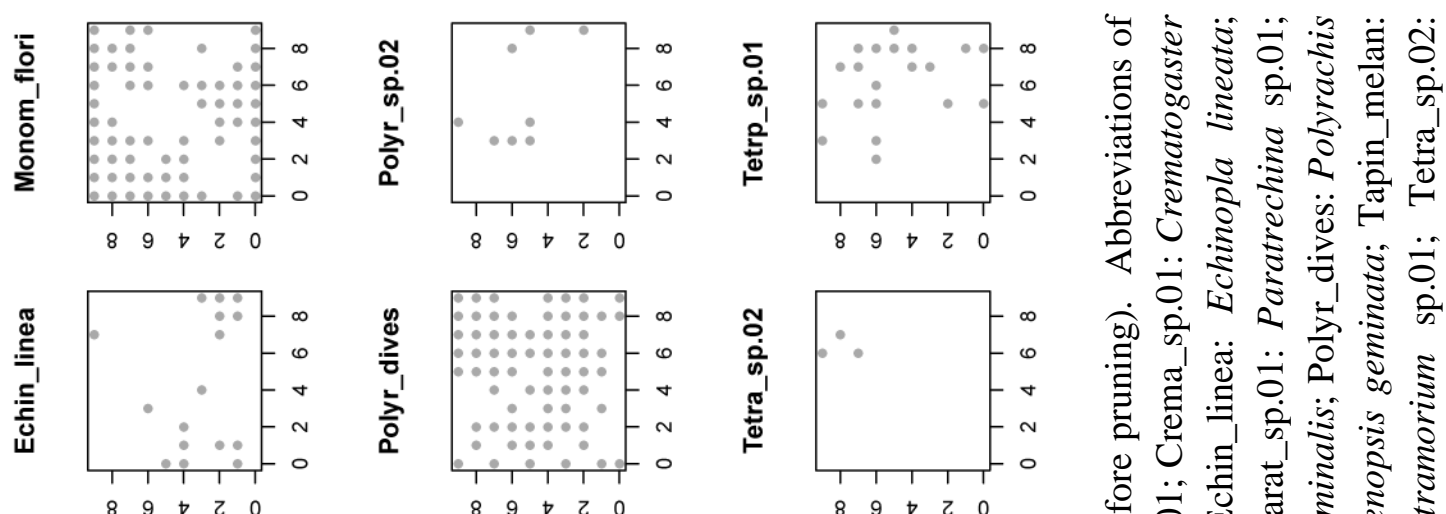

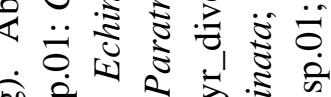

की

党 离

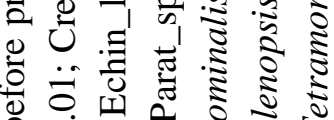

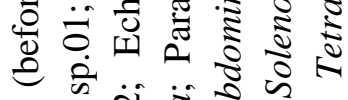
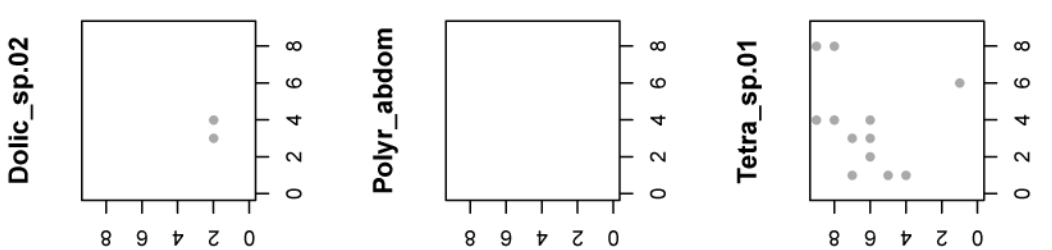

के

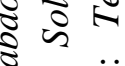

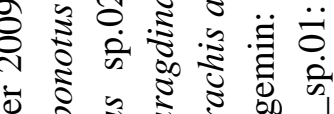

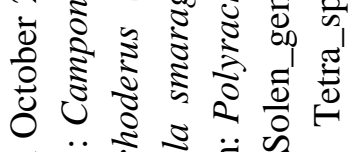

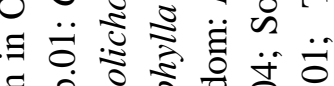
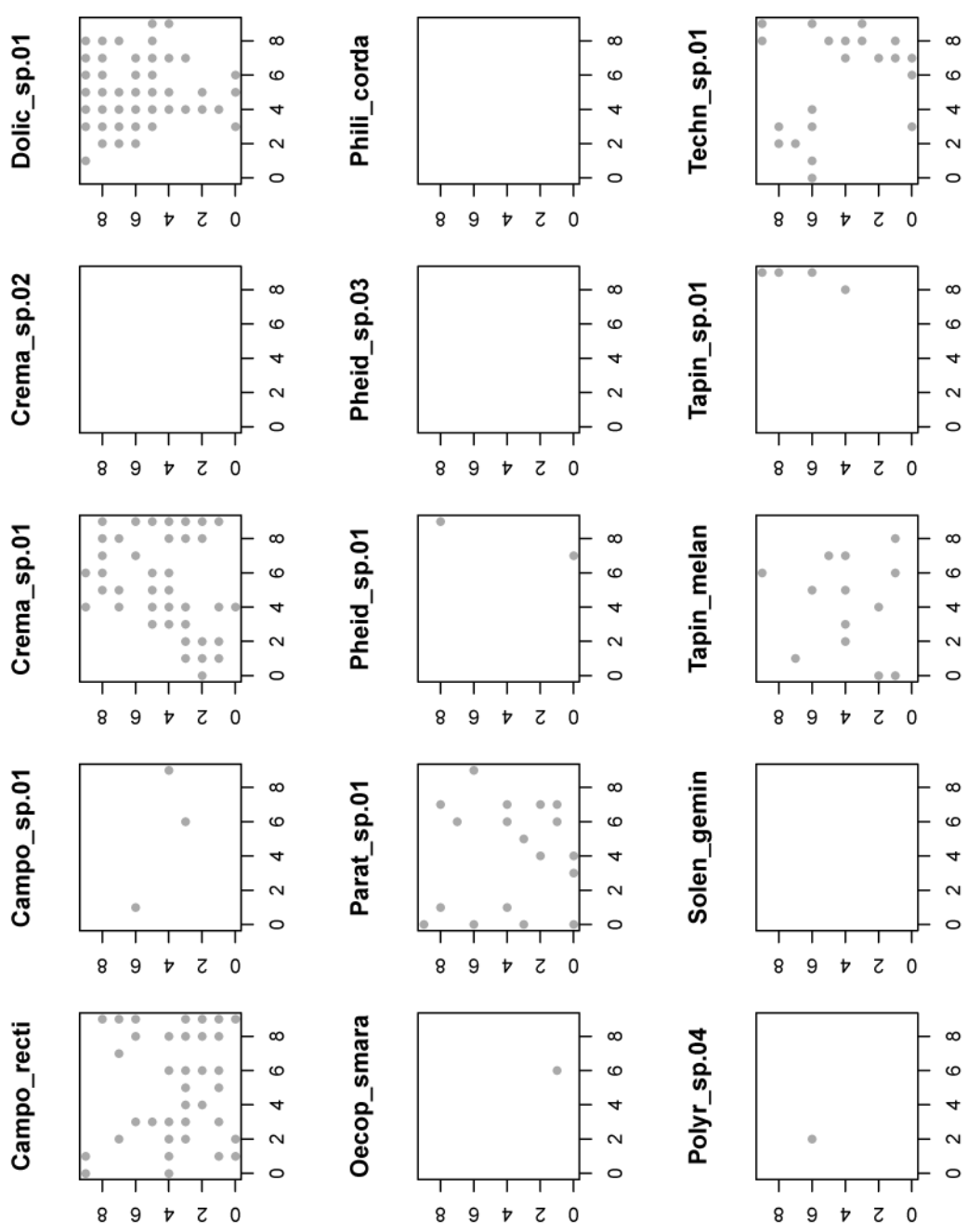

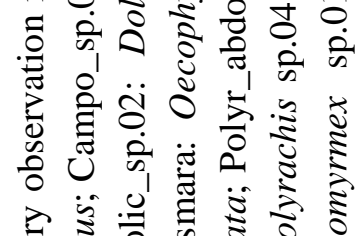

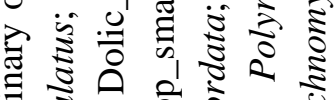

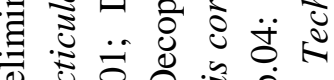

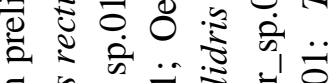

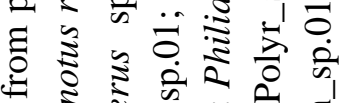

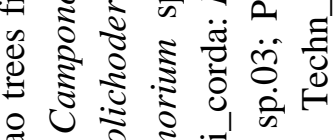

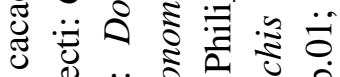

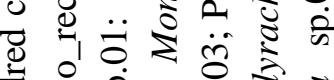

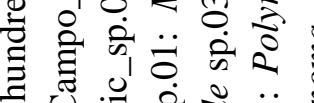
๙

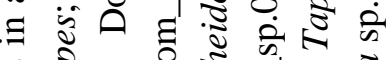

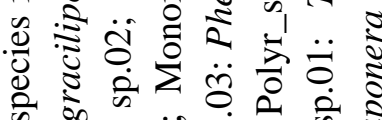
क्र कo की

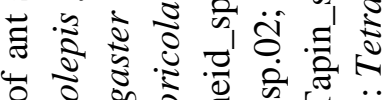
प० 50

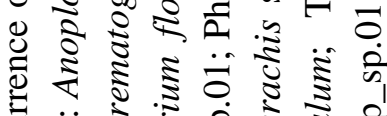

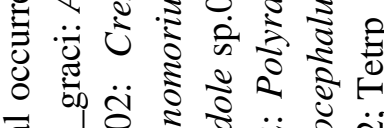
चี
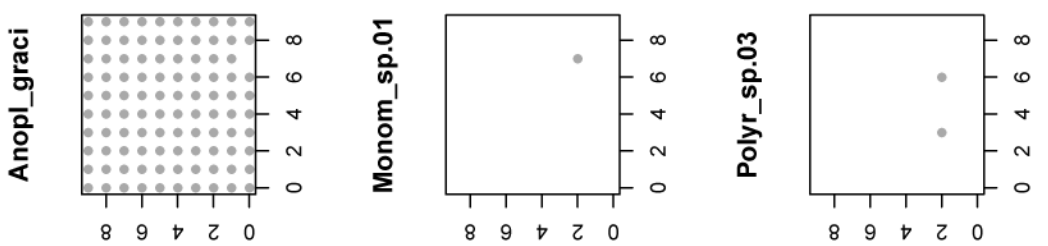
की की के के को

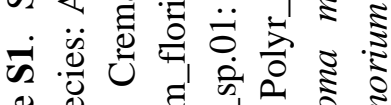

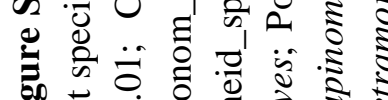

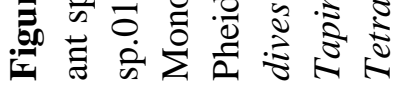



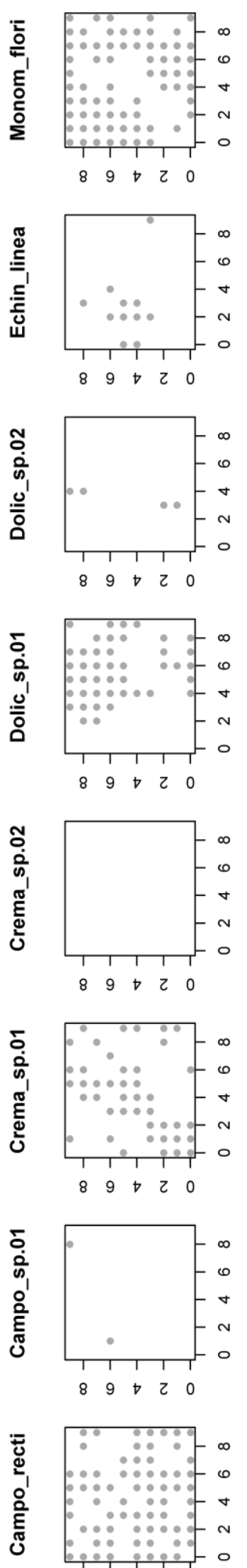

$89 \ni 20$

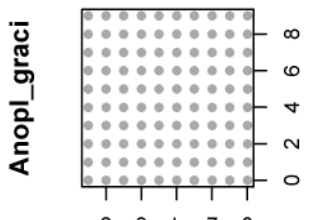

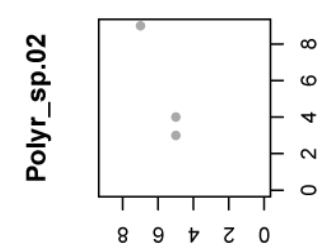
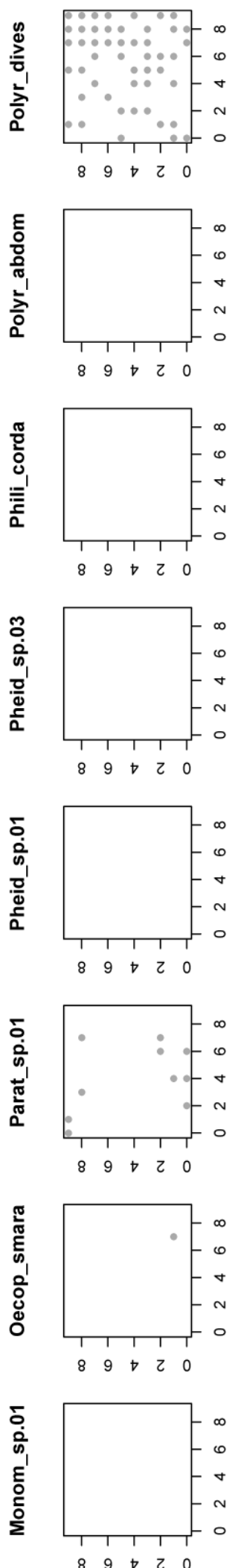
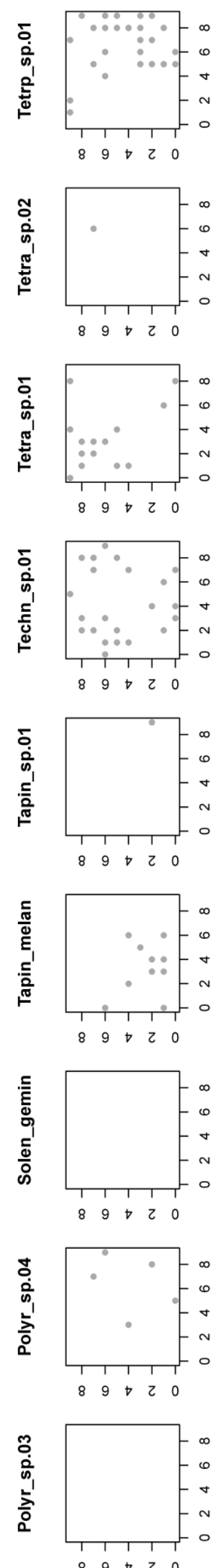

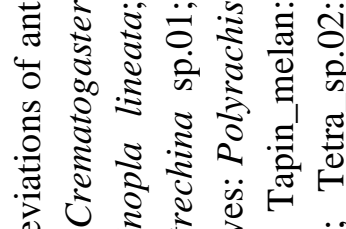

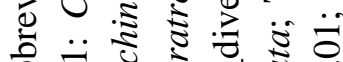

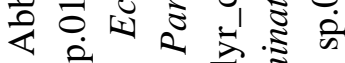

की की

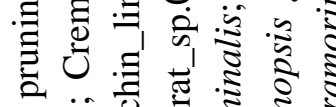

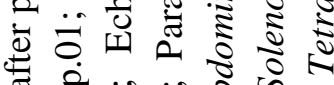

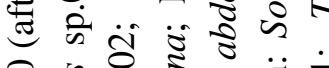

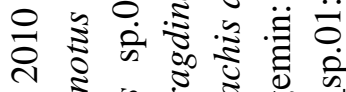

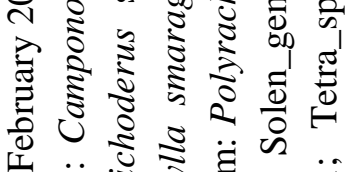

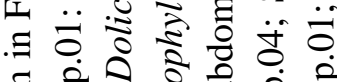

क की हु के के के

营造它

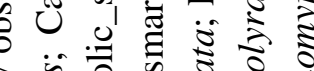

उิ

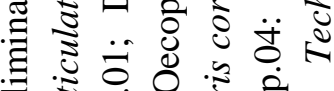

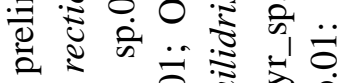

है

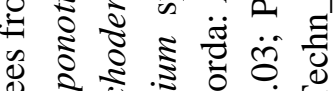

ग)

ஆ

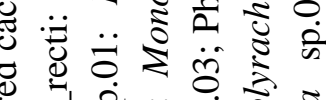

至 0 को

है है

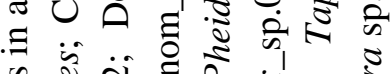

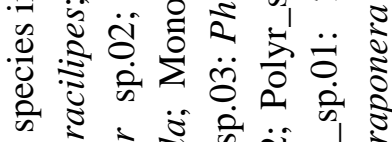

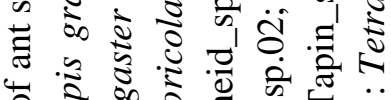

पे 0

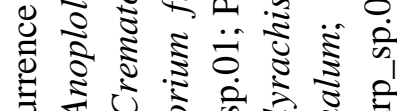

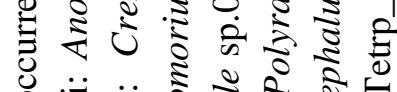

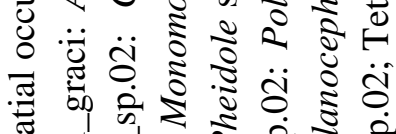

है का रे के के

क

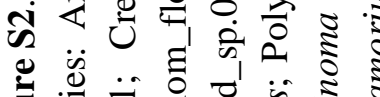

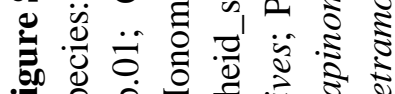



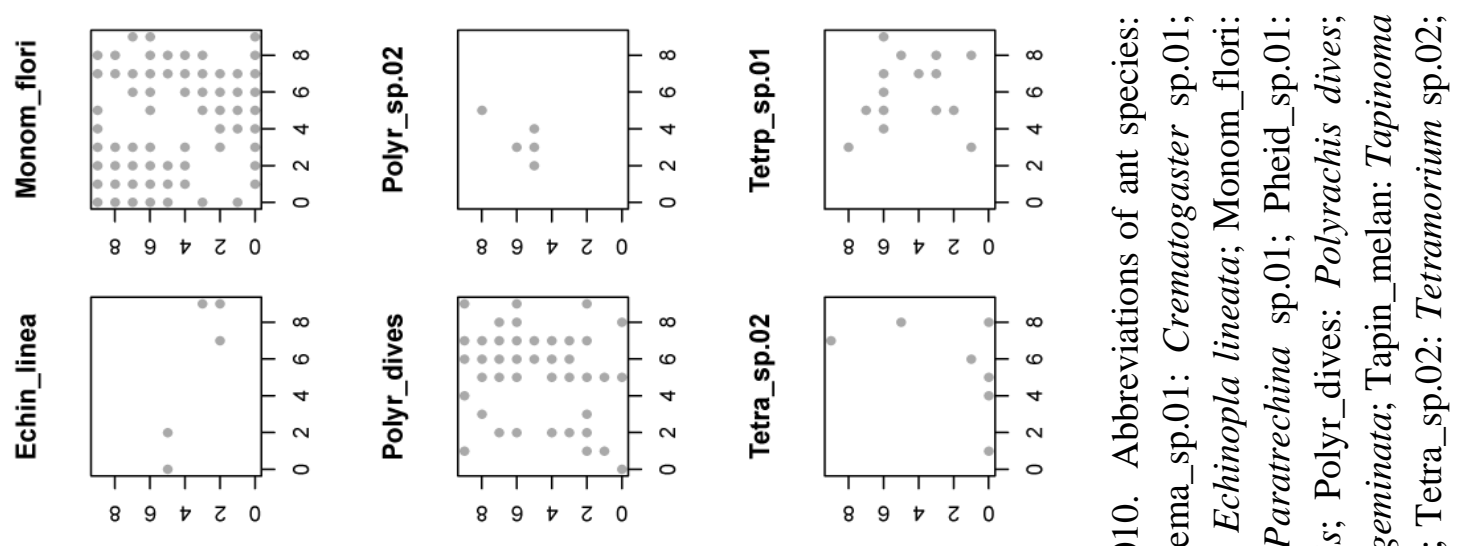

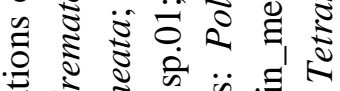
.

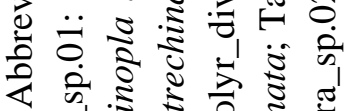

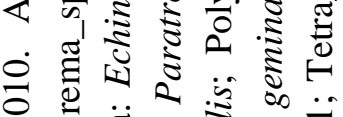
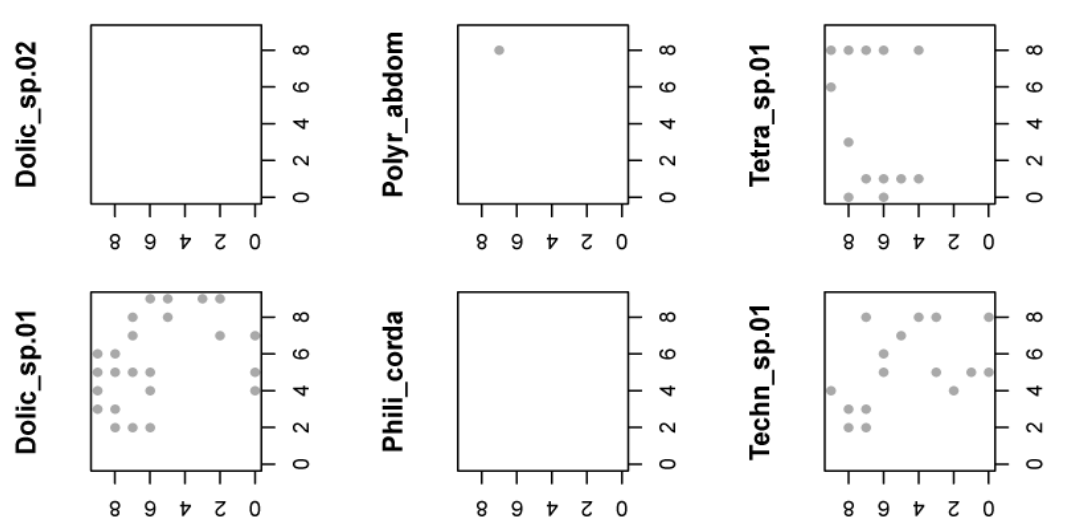

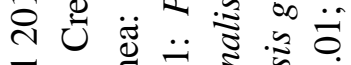

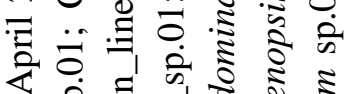

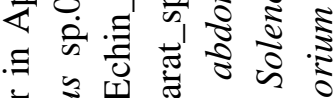

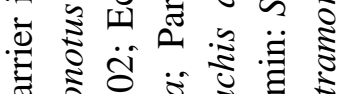

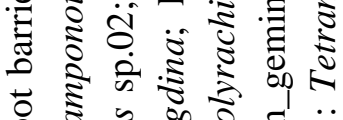

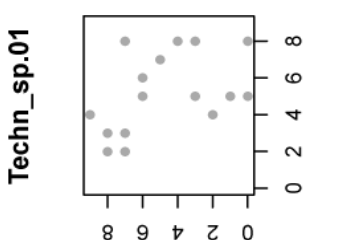

ㅇำ

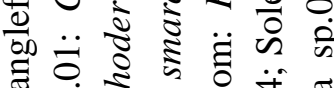

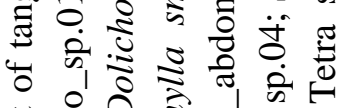
言 范
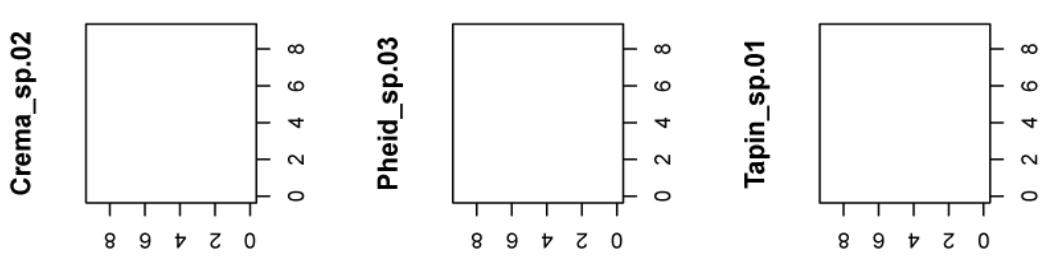

च ป

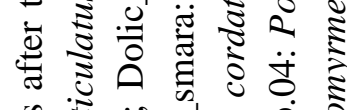
ठँ
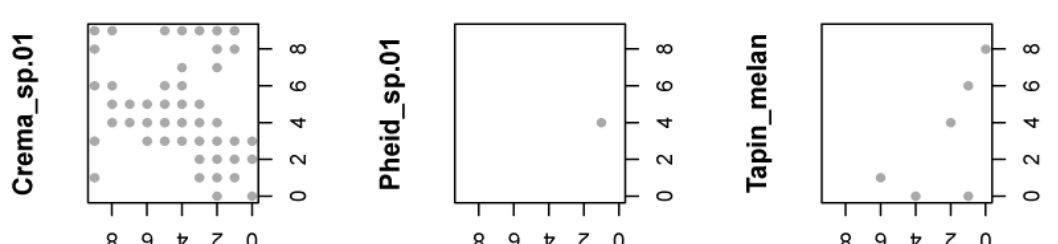

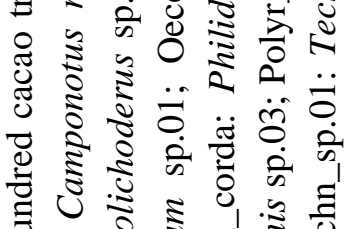

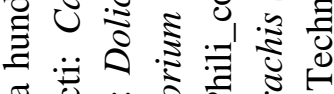
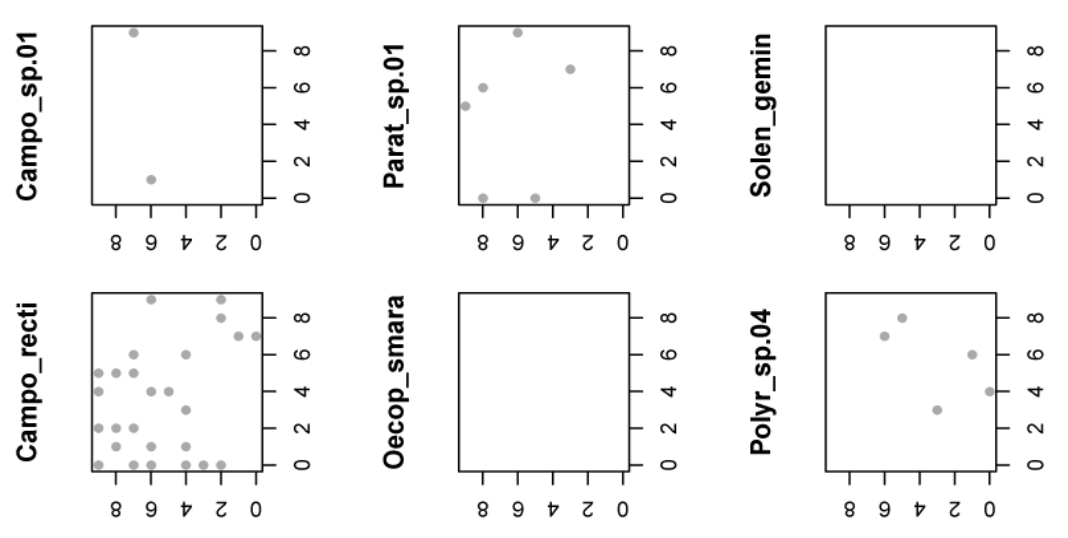

$\Xi \mathscr{U}_{1} \ddot{\bar{\sigma}} \cong$

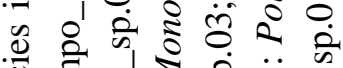

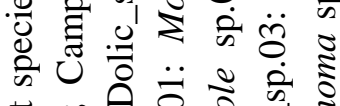
ज行

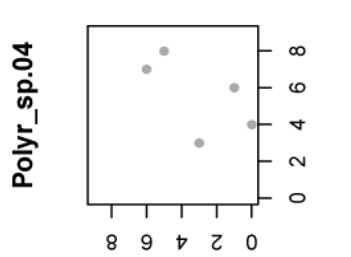
पे :

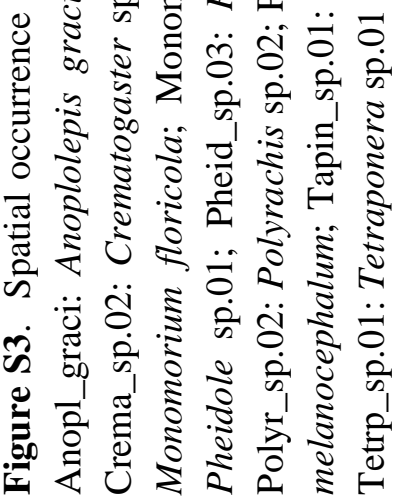




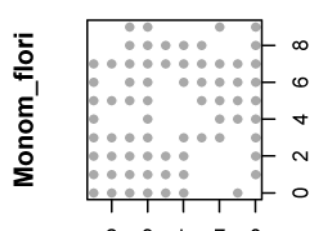

$89 \forall z$
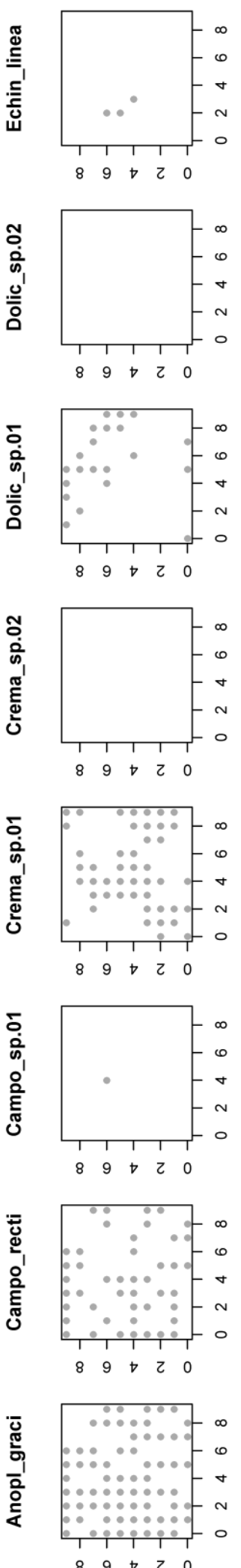
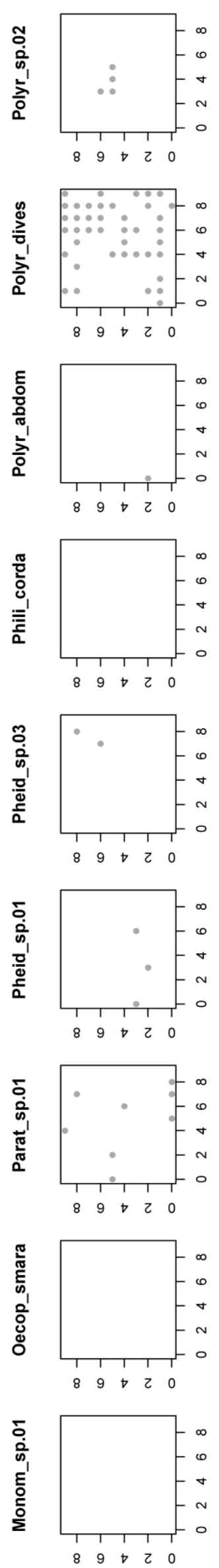
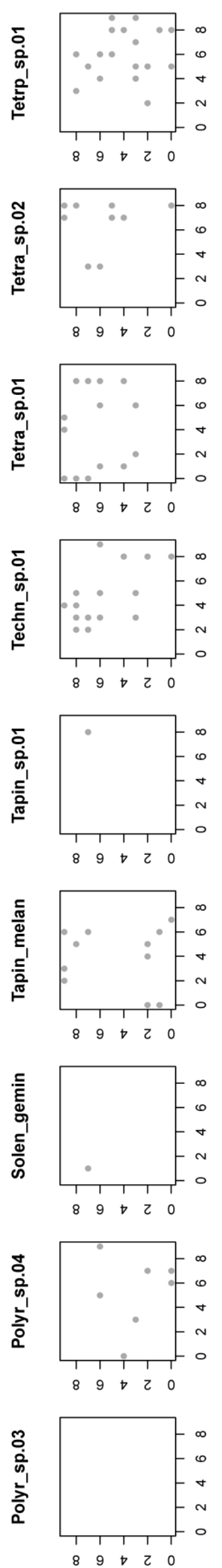

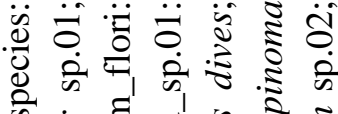

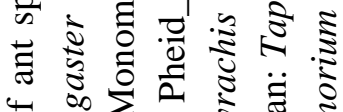
$4 \sum_{0}^{\infty} \sum \ldots$ 苛 . ज गें

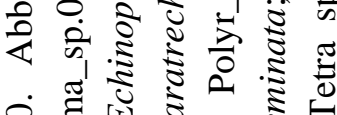

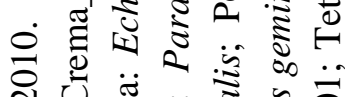
๙U.

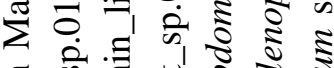
च की चु

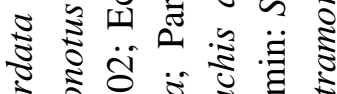

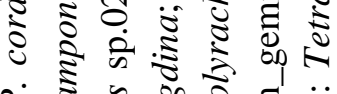

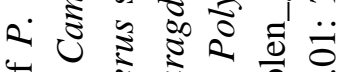
4..

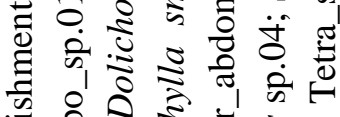
产 एँ

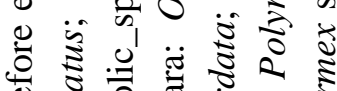
ग 总芯范 अ

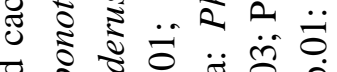

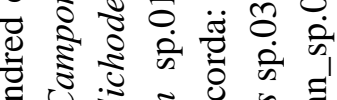

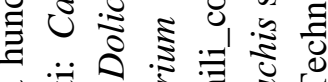
ब

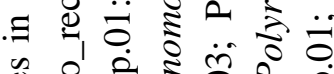
. 造 䒕

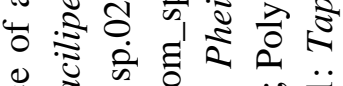

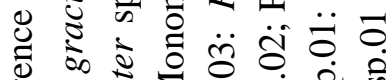
ह

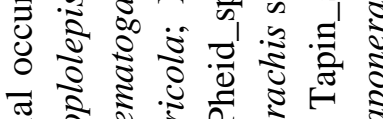
శี की

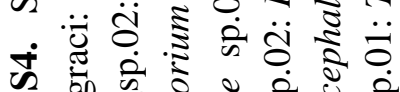
का का के ई

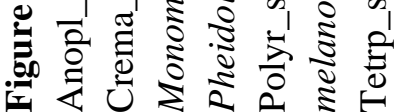



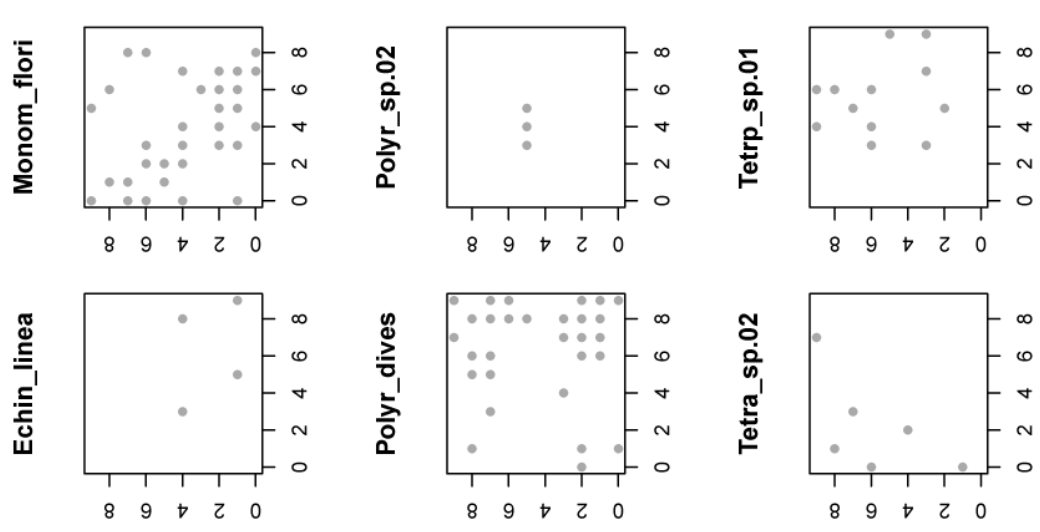

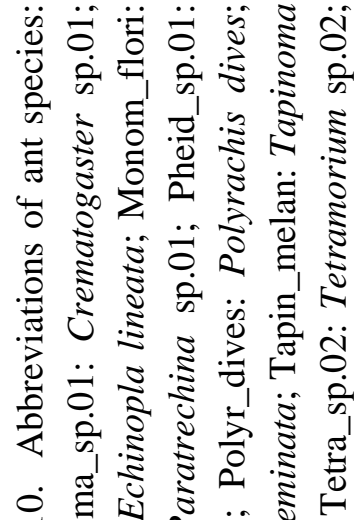
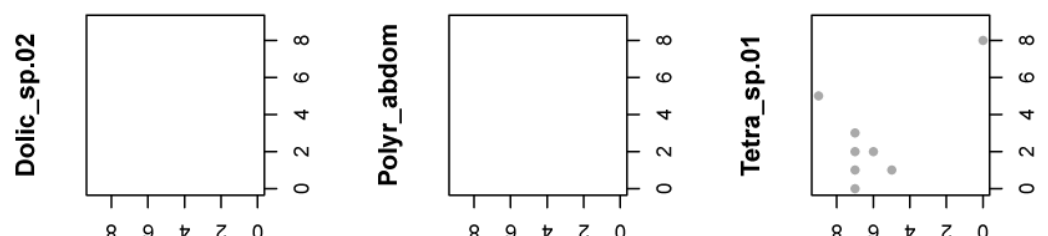

글
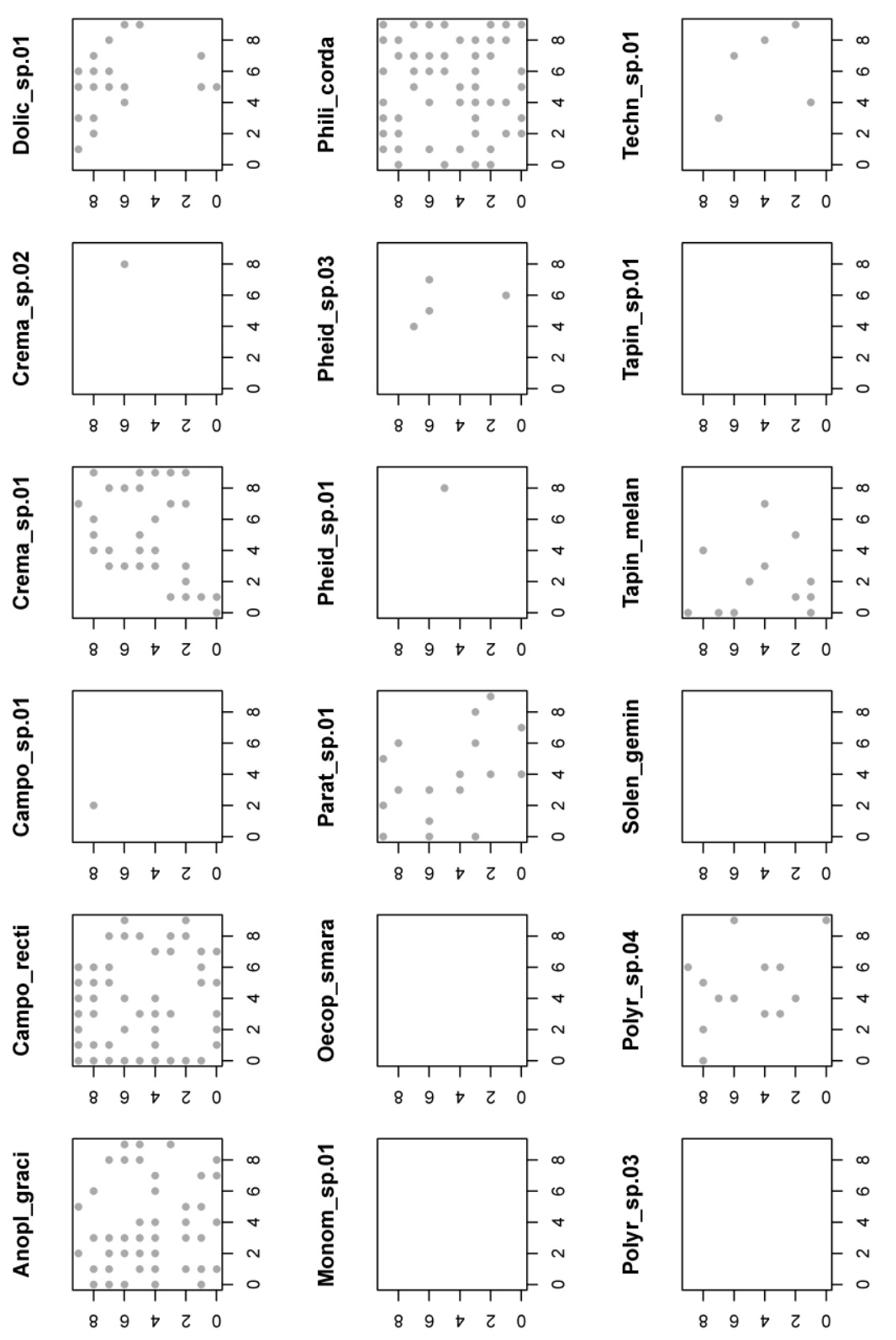

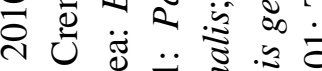

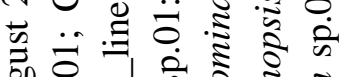

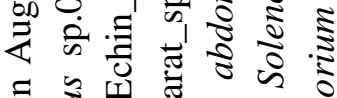

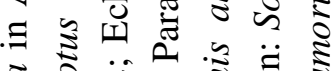

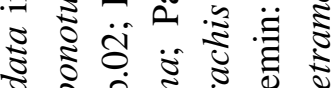

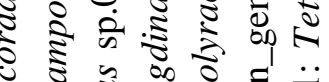

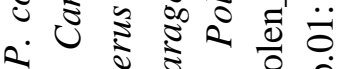

पें

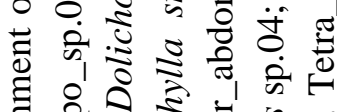

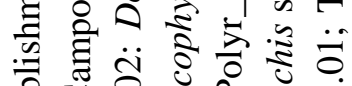

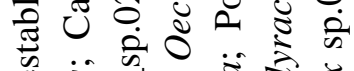

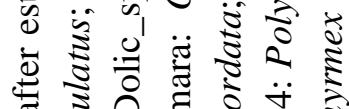

के

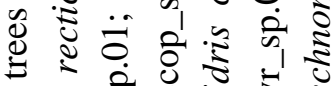

ॠ के

ठ

चु

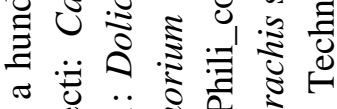

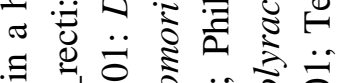

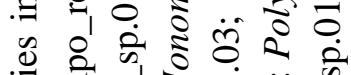

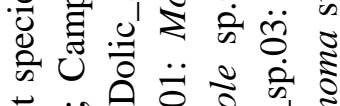

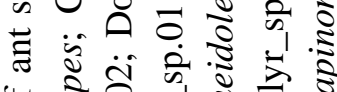

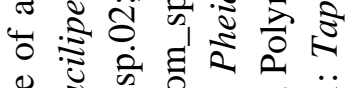

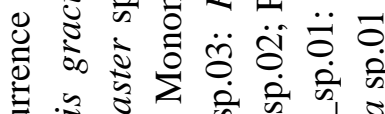

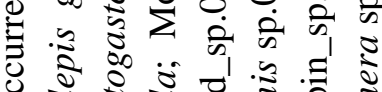

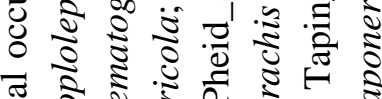
न

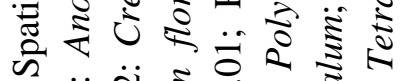

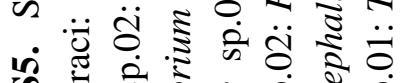

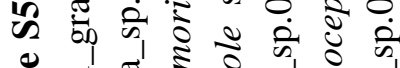

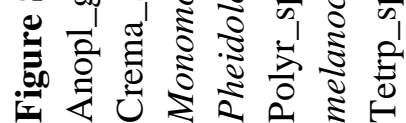




\section{SUMMARY}

Land-use and climate change are the most important driver of biodiversity loss in terrestrial ecosystems. Numerous studies show how land-use change, which means in many cases conversion of natural habitat to agriculture, severely affects biodiversity. Furthermore, climate change through temperature increase and reduced precipitation has an effect on distribution, reproduction and behavior of organisms. In addition, intensification of agricultural management through the conversion to monocultures and pesticide application have further negative impact on diversity in agricultural habitats. However, some agricultural land-use types are able to conserve biodiversity. For instance, shade-trees in agroforestry system reduce pressure on habitat destruction and also from climate change. Unfortunately, information about long-term patterns of biodiversity in agroecosystems, including agroforests, is lacking. The species composition and the species richness of communities in transformed ecosystems may not be stable even if management does not change. Therefore, an adequate assessment of the value of agroforests for conservation would benefit from insights gained through the analysis of long-term datasets.

In this research, we studied ant community structure and biological control in cacao agroforestry with emphasized on the effect of long-term changes, land-use management and precipitation gradients. We addressed the following questions: how does land-use management and precipitation gradients affect ant diversity in cacao agroforestry systems? (chapter 2), how does ant diversity change over several years in cacao agroforestry systems? (chapter 3), and how does the species richness of ants, ant the identity of dominant ant species affect pests and diseases in cacao agroforestry systems? (chapter 4). The research was conducted in cacao agroforestry located around Lore Lindu National Park (LLNP), Central Sulawesi, Indonesia. We sampled ants in sixteen plots spread in 
eight villages, representing different of land-use management and gradients of precipitation, to study the effect of land-use management and precipitation gradients on ant diversity (chapter 2). We resampled ants in 2009 with the same method and in the same sites that have been previously studied in 2001 and 2003 within the STORMA (Stability of Rainforest Margins in Indonesia) research project, to study the long-term change of ant diversity in cacao agroforestry (chapter 3). To study biological control provided by ants, we conducted field experiment to separate the effect of ant species identity and species richness on pests and diseases (chapter 4).

Our results showed that decreasing numbers of shade trees have no effect on ant diversity in cacao agroforestry on a regional scale. In contrast, precipitation strongly influences ant community similarity in cacao agroforestry (chapter 2). The long-term change in ant communities differed greatly between rainforest-poor (Palolo region) and rainforest-rich (Kulawi region). Temporal changes in ant communities in agroforestry appeared to be more difficult to predict than expected, highlighting the importance of longterm studies for better understanding of the processes underlying the patterns. Our findings suggest that the landscape-scale differences between the two study regions play a more important role than changes in local management. The rainforest-rich region appeared to support a rich species pool with ants colonizing cacao agroforestry and thereby compensating for local land-use intensification (chapter 3). Finally, ant species richness had no significant effect on pest incidence or in improving detection of prey in the predation experiment, and was not associated with a reduction in the incidence of a minor pest. In contrast, incidence of the disease caused by $P$. palmivora was higher in ant species rich trees. Identity of the dominant ant species affected incidence of pests and diseases as well as predation pressure. Both ant species richness and identity are associated with ecosystem services and disservices delivered by the ant community (chapter 4). 
In conclusion, climate change in terms of reduced precipitation has the potential to shape ant communities in cacao agroforestry. Cacao agroforestry, which is surrounded by high quality habitat such as a rainforest reserve can still sustain ant biodiversity, spilling over to land-use systems and protecting land use from long-term effect of agricultural intensification and environmental change. In cacao agroforestry, ants provide an essential role for ecosystem services such as biological control based on both the identity of individual species and the overall ant species richness. 


\section{ACKNOWLEDGEMENTS}

I would like to express my gratitude to Prof. Dr. Teja Tscharntke and Dr. Yann Clough for their thorough, but flexible supervision during the last three years and for their important efforts for research funding. I learned a lot from them and I am very thankful for the opportunity they gave me to develop my skills in setting up field research and writing scientific manuscripts.

I sincerely thank to Dr. Damayanti Buchori for her support and important efforts for research funding. I am very thankful to Arno Wielgoss for his support and share his experience during fieldwork in Palu, Sulawesi. Thanks to Adha Sari, Putri Syahierah and Yuanita for their support during laboratory work in Bogor Agricultural University (IPB). Thanks also go to staff of the Center for Tropical Forest Margin (CTFM), University of Tadulako (UNTAD) especially Dr. Abdul Rauf and mbak Anti for supporting the administration and transportation during field work.

The following persons were very important to me during the field research in Sulawesi: (i) field assistants: Fifin, Moh. Fadlan, Akhmad Syakur, and Hadiprayitno, (ii) laboratory assistants: Nur Ivon and Dewi Utaminingsih, (iii) drivers of the CTFM: Pak Alex, Pak Gauk and Pak Baso, (iv) local assistants: Ahmad, Boni, Baswan, Alfianus and Mardianus, and (v) students from UNTAD: M. Khaidir, Indrawan, M. Rizki, Laode, and Rizal. Thanks also to PT. Sucofindo (Palu Branch Office) especially Pak Nyoman who gave permission to use the fogging machine.

During my stay in Sulawesi, I always enjoyed the hospitality of the people of the Ranteleda village, Palolo (Pak Imam, Pak Ahmad and Bu Salmin) and the Boladangko village, Kulawi (Pak Roma and Pak Hamzah). I also thank in particular the owners of study sites: Berdikari village (Yosias Nthu and Yosep Sapangan), Ranteleda village 
(Baharudin), Sintuwu village (Tahana M. Suari and Iskandar), Bulili village (Sofyan and Tahang), Pandere village (Kunci and Nusrin Abd. Samad), Sidondo village (Asran Hadoha and H. Manno), Boladangko village (Hamzah and Toruba), Toro village (Faldin), and Lempelero village (Mursan and Pohabe).

I really want to thank my friends and colleagues in the Agroecology group for their help, discussions, inspiration, support, and for having a great time with good friends in Göttingen. Thanks to Jutta Gilles, Susanne Jahn and Susanne Schiele for their support during my $\mathrm{PhD}$ work in the Agroecology group. I also thanks to all of Indonesian friends in Göttingen especially member of PPI Göttingen and KALAM who support during my study in Göttingen.

I deeply thank to my wife, Sudi Herlin Rahmawati for her support, encouragement, trust and enthusiasm and for being around at all good and difficult times during the field work in Sulawesi and my $\mathrm{PhD}$ work in Göttingen.

This research project was funded by the German Science Foundation (DFG), University of Göttingen, Bogor Agricultural University, and Directorate General of Higher Education (DIKTI), Ministry of National Education of the Republic of Indonesia. 


\section{LIST OF PUBLICATIONS}

Rizali A., Clough Y., Buchori D., Hosang M.L.A., Bos M.M., Tscharntke T. (2012) Longterm change of ant community structure in cacao agroforestry landscapes in Indonesia. Insect Conservation and Diversity, DOI: 10.1111/j.1752-4598.2012. 00219.x

Rizali A., Rahim A., Sahari B., Prasetyo L.B., Buchori D. (2011) Impact of invasive ant species in shaping ant community structure on small islands in Indonesia. Indonesian Journal of Biology (Jurnal Biologi Indonesia), 7, 221-230.

Herlina N., Rizali A., Moerfiah, Sahari B., Buchori D. (2011) Effect of rice field surrounding habitat and age of rice plant on the diversity of Parasitic Hymenoptera. Indonesian Journal of Entomology, 8, 17-26.

Setiani E.A., Rizali A., Moerfiah, Sahari B., Buchori D. (2010) Ant diversity in rice field in urban landscape: investigation on the effect of habitat condition and age of rice plant. Indonesian Journal of Entomology, 7, 88-99.

Rizali A., Lohman D.J., Buchori D., Prasetyo L.B., Triwidodo H., Bos M.M., Yamane S., Schulze C.H. (2010) Ant communities on small, disturbed tropical islands: effects of island size and isolation are obscured by habitat disturbance and 'tramp ant species'. Journal of Biogeography, 37, 229-236.

Sahari B., Rizali A., Buchori D. (2010) Insect pollinator communities under changing land-use in tropical landscapes: implications for agricultural management in Indonesia. Tropical Rainforests and Agroforests under Global Change: Ecological and Socio-economic Valuation. (ed. by T. Tscharntke, C. Leuschner, E. Veldkamp, H. Faust, E. Guharja, A. Bidin), pp. 97-114. Springer-Verlag, Berlin 
Rizali A., Bos M.M., Buchori D., Yamane S., Schulze C.H. (2008) Ants in tropical urban habitats: The myrmecofauna in a densely populated area in West Java, Indonesia. HAYATI Journal of Bioscience, 15, 77-84.

Rizali A. (2003) Research Plan: Ant diversity in a human-modified landscape - a case study from Bogor, West Java Indonesia. ANeT Newsletter, 5, 12-13.

Rizali A. (2002) Preliminary study of the ants subfamily Ponerinae (Hymenoptera: Formicidae) in Cikaniki, Gunung Halimun National Park. Tropical Ecology and Biodiversity (Ekologi \& Biodiversitas Tropika), 1, 21-33.

Rizali A., Buchori D., Triwidodo H. (2002) Insect diversity at the forest margin-rice field interface: indicator for a healthy ecosystem. HAYATI Journal of Bioscience, 9, 4148. 


\title{
CURRICULUM VITAE
}

\author{
Akhmad Rizali \\ Born 15.04.1977 in Madiun, Indonesia
}

\section{Education}

2008 - 2011 PhD study. Thesis: "Ant community structure and biological control in Indonesian cacao agroforestry: long-term changes, land-use management and precipitation gradient”. Agroecology, Department of Crop Sciences. University of Göttingen, Germany.

2003 - 2006 MSc study. Thesis: "Ant diversity in Seribu Island, Indonesia" at the Entomology Program, Department of Plant Protection, Bogor Agricultural University.

1995 - 2000 BSc study. Thesis: "Insect diversity and its role at rice field in Gunung Halimun National Park, Bogor District, West Java" at Department of Plant Protection, Bogor Agricultural University.

1992 - 1995 Senior High School at SMAN 1 Wungu, Madiun

1989 - 1992 Junior High School at SMPN 1 Wungu, Madiun

1983 - 1989 Elementary School at SDN 1 Wungu, Madiun 